\title{
The Foldy-Lax approximation of the scattered waves by many small bodies for the Lamé system
}

\author{
Durga Prasad Challa*† Mourad Sini ${ }^{\ddagger}$
}

October 8, 2018

\begin{abstract}
We are concerned with the linearized, isotropic and homogeneous elastic scattering problem by many small rigid obstacles of arbitrary, Lipschitz regular, shapes in 3D case. We prove that there exists two constant $a_{0}$ and $c_{0}$, depending only on the Lipschitz character of the obstacles, such that under the conditions $a \leq a_{0}$ and $\sqrt{M-1} \frac{a}{d} \leq c_{0}$ on the number $M$ of the obstacles, their maximum diameter $a$ and the minimum distance between them $d$, the corresponding Foldy-Lax approximation of the farfields is valid. In addition, we provide the error of this approximation explicitly in terms of the three parameters $M, a$ and $d$. These approximations can be used, in particular, in the identification problems (i.e. inverse problems) and in the design problems (i.e. effective medium theory).
\end{abstract}

Keywords: Elastic wave scattering, Small-scatterers, Foldy-Lax approximation, Capacitance.

\section{Introduction and statement of the results}

Let $B_{1}, B_{2}, \ldots, B_{M}$ be $M$ open, bounded and simply connected sets in $\mathbb{R}^{3}$ with Lipschitz boundaries, containing the origin. We assume that their sizes and Lipschitz constants are uniformly bounded. We set $D_{m}:=\epsilon B_{m}+z_{m}$ to be the small bodies characterized by the parameter $\epsilon>0$ and the locations $z_{m} \in \mathbb{R}^{3}$, $m=1, \ldots, M$.

Assume that the Lamé coefficients $\lambda$ and $\mu$ are constants satisfying $\mu>0$ and $3 \lambda+2 \mu>0$. Let $U^{i}$ be a solution of the Navier equation $\left(\Delta^{e}+\omega^{2}\right) U^{i}=0$ in $\mathbb{R}^{3}, \Delta^{e}:=(\mu \Delta+(\lambda+\mu) \nabla$ div $)$. We denote by $U^{s}$ the elastic field scattered by the $M$ small bodies $D_{m} \subset \mathbb{R}^{3}$ due to the incident field $U^{i}$. We restrict ourselves to the scattering by rigid bodies. Hence the total field $U^{t}:=U^{i}+U^{s}$ satisfies the following exterior Dirichlet problem of the elastic waves

$$
\begin{gathered}
\left(\Delta^{e}+\omega^{2}\right) U^{t}=0 \text { in } \mathbb{R}^{3} \backslash\left(\bigcup_{m=1}^{M} \bar{D}_{m}\right), \\
\left.U^{t}\right|_{\partial D_{m}}=0,1 \leq m \leq M
\end{gathered}
$$

with the Kupradze radiation conditions (K.R.C)

$$
\lim _{|x| \rightarrow \infty}|x|\left(\frac{\partial U_{p}}{\partial|x|}-i \kappa_{p} U_{p}\right)=0, \text { and } \lim _{|x| \rightarrow \infty}|x|\left(\frac{\partial U_{s}}{\partial|x|}-i \kappa_{s} \omega U_{s}\right)=0,
$$

where the two limits are uniform in all the directions $\hat{x}:=\frac{x}{|x|} \in \mathbb{S}^{2}$. Also, we denote $U_{p}:=-\kappa_{p^{\omega}}^{-2} \nabla\left(\nabla \cdot U^{s}\right)$ to be the longitudinal (or the pressure or $\mathrm{P}$ ) part of the field $U^{s}$ and $U_{s}:=\kappa_{s \omega}^{-2} \nabla \times\left(\nabla \times U^{s}\right)$ to be

\footnotetext{
${ }^{*}$ Corresponding author : Durga Prasad Challa

${ }^{\dagger}$ RICAM, Austrian Academy of Sciences, Altenbergerstrasse 69, A-4040, Linz, Austria. Email:durga.challa@oeaw.ac.at, Tel: +43 (0)732 2468 5234. Supported by the Austrian Science Fund (FWF): P22341-N18.

${ }^{\ddagger}$ RICAM, Austrian Academy of Sciences, Altenbergerstrasse 69, A-4040, Linz, Austria. Email:mourad.sini@oeaw.ac.at, Tel: +43 (0)732 2468 5258. Partially supported by the Austrian Science Fund (FWF): P22341-N18.
} 
the transversal (or the shear or $\mathrm{S}$ ) part of the field $U^{s}$ corresponding to the Helmholtz decomposition $U^{s}=U_{p}+U_{s}$. The constants $\kappa_{p^{\omega}}:=\frac{\omega}{c_{p}}$ and $\kappa_{s} \omega:=\frac{\omega}{c_{s}}$ are known as the longitudinal and transversal wavenumbers, $c_{p}:=\sqrt{\lambda+2 \mu}$ and $c_{s}:=\sqrt{\mu}$ are the corresponding phase velocities, respectively and $\omega$ is the frequency.

The scattering problem (1.1, 1.3) is well posed in the Hölder or Sobolev spaces, see [12, 13, 16, 17, for instance, and the scattered field $U^{s}$ has the following asymptotic expansion:

$$
U^{s}(x):=\frac{e^{i \kappa_{p} \omega}|x|}{|x|} U_{p}^{\infty}(\hat{x})+\frac{e^{i \kappa_{s} \omega|x|}}{|x|} U_{s}^{\infty}(\hat{x})+O\left(\frac{1}{|x|^{2}}\right),|x| \rightarrow \infty
$$

uniformly in all directions $\hat{x} \in \mathbb{S}^{2}$. The longitudinal part of the far-field, i.e. $U_{p}^{\infty}(\hat{x})$ is normal to $\mathbb{S}^{2}$ while the transversal part $U_{s}^{\infty}(\hat{x})$ is tangential to $\mathbb{S}^{2}$. As usual in scattering problems we use plane incident waves in this work. For the Lamé system, the full plane incident wave is of the form $U^{i}(x, \theta):=\alpha \theta e^{i \kappa_{p} \omega \theta \cdot x}+\beta \theta^{\perp} e^{i \kappa_{s} \omega \theta \cdot x}$, where $\theta^{\perp}$ is any direction in $\mathbb{S}^{2}$ perpendicular to the incident direction $\theta \in \mathbb{S}^{2}, \alpha, \beta$ are arbitrary constants. In particular, the pressure and shear incident waves are $U^{i, p}(x, \theta):=\theta e^{i \kappa_{p} \omega \theta \cdot x}$ and $U^{i, s}(x, \theta):=\theta^{\perp} e^{i \kappa_{s} \omega \theta \cdot x}$, respectively. Pressure incident waves propagate in the direction of $\theta$, whereas shear incident waves propagate in the direction of $\theta^{\perp}$. The functions $U_{p}^{\infty}(\hat{x}, \theta):=U_{p}^{\infty}(\hat{x})$ and $U_{s}^{\infty}(\hat{x}, \theta):=U_{s}^{\infty}(\hat{x})$ for $(\hat{x}, \theta) \in \mathbb{S}^{2} \times \mathbb{S}^{2}$ are called the $\mathrm{P}$ part and the $\mathrm{S}$ part of the far-field pattern respectively.

\section{Definition 1.1. We define}

1. a as the maximum among the diameters, diam, of the small bodies $D_{m}$, i.e.

$$
a:=\max _{1 \leq m \leq M} \operatorname{diam}\left(D_{m}\right) \quad\left[=\epsilon \max _{1 \leq m \leq M} \operatorname{diam}\left(B_{m}\right)\right]
$$

2. $d$ as the minimum distance between the small bodies $\left\{D_{1}, D_{2}, \ldots, D_{m}\right\}$, i.e.

$$
d:=\min _{\substack{m \neq j \\ 1 \leq m, j \leq M}} d_{m j}
$$

where $_{m j}:=\operatorname{dist}\left(D_{m}, D_{j}\right)$. We assume that

$$
0<d \leq d_{\max }
$$

and $d_{\max }$ is given.

3. $\omega_{\max }$ as the upper bound of the used frequencies, i.e. $\omega \in\left[0, \omega_{\max }\right]$.

4. $\Omega$ to be a bounded domain in $\mathbb{R}^{3}$ containing the small bodies $D_{m}, m=1, \ldots, M$.

The main result of this paper is the following theorem.

Theorem 1.2. There exist two positive constants $a_{0}$ and $c_{0}$ depending only on the size of $\Omega$, the Lipschitz character of $B_{m}, m=1, \ldots, M, d_{\max }$ and $\omega_{\max }$ such that if

$$
a \leq a_{0} \text { and } \sqrt{M-1} \frac{a}{d} \leq c_{0}
$$

then the P-part, $U_{p}^{\infty}(\hat{x}, \theta)$, and the $S$-part, $U_{s}^{\infty}(\hat{x}, \theta)$, of the far-field pattern have the following asymptotic expressions

$$
U_{p}^{\infty}(\hat{x}, \theta)=\frac{1}{4 \pi c_{p}^{2}}(\hat{x} \otimes \hat{x})\left[\sum_{m=1}^{M} e^{-i \frac{\omega}{c_{p}} \hat{x} \cdot z_{m}} Q_{m}+O\left(M a^{2}+M(M-1) \frac{a^{3}}{d^{2}}+M(M-1)^{2} \frac{a^{4}}{d^{3}}\right)\right],
$$




$$
U_{s}^{\infty}(\hat{x}, \theta)=\frac{1}{4 \pi c_{s}^{2}}(I-\hat{x} \otimes \hat{x})\left[\sum_{m=1}^{M} e^{-i \frac{\omega}{c_{s}} \hat{x} \cdot z_{m}} Q_{m}+O\left(M a^{2}+M(M-1) \frac{a^{3}}{d^{2}}+M(M-1)^{2} \frac{a^{4}}{d^{3}}\right)\right]
$$

uniformly in $\hat{x}$ and $\theta$ in $\mathbb{S}^{2}$. The constant appearing in the estimate $O($.$) depends only on the size of \Omega$, the Lipschitz character of the reference bodies $B_{m}, a_{0}, c_{0}$ and $\omega_{\text {max }}$. The vector coefficients $Q_{m}, m=1, \ldots, M$, are the solutions of the following linear algebraic system

$$
C_{m}^{-1} Q_{m}=-U^{i}\left(z_{m}, \theta\right)-\sum_{\substack{j=1 \\ j \neq m}}^{M} \Gamma^{\omega}\left(z_{m}, z_{j}\right) Q_{j}
$$

for $m=1, \ldots, M$, with $\Gamma^{\omega}$ denoting the Kupradze matrix of the fundamental solution to the Navier equation with frequency $\omega, C_{m}:=\int_{\partial D_{m}} \sigma_{m}(s) d s$ and $\sigma_{m}$ is the solution matrix of the integral equation of the first kind

$$
\int_{\partial D_{m}} \Gamma^{0}\left(s_{m}, s\right) \sigma_{m}(s) d s \quad=\quad \boldsymbol{I}, \mathrm{s}_{\mathrm{m}} \in \partial \mathrm{D}_{\mathrm{m}}
$$

with $\boldsymbol{I}$ the identity matrix of order 3. The algebraic system (1.10) is invertible under the condition:

$$
\frac{a}{d} \leq c_{1} t^{-1}
$$

with

$$
t:=\left[\frac{1}{c_{p}^{2}}-2 \operatorname{diam}(\Omega) \frac{\omega}{c_{s}^{3}}\left(\frac{1-\left(\frac{1}{2} \kappa_{s} \omega \operatorname{diam}(\Omega)\right)^{N_{\Omega}}}{1-\left(\frac{1}{2} \kappa_{s} \omega \operatorname{diam}(\Omega)\right)}+\frac{1}{2^{N_{\Omega}-1}}\right)-\operatorname{diam}(\Omega) \frac{\omega}{c_{p}^{3}}\left(\frac{1-\left(\frac{1}{2} \kappa_{p} \omega \operatorname{diam}(\Omega)\right)^{N_{\Omega}}}{1-\left(\frac{1}{2} \kappa_{p} \omega \operatorname{diam}(\Omega)\right)}+\frac{1}{2^{N_{\Omega^{-1}}}}\right)\right],
$$

which is assumed to be positiv 1$]$ and $N_{\Omega}:=\left[2 \operatorname{diam}(\Omega) \max \left\{\kappa_{s^{\omega}}, \kappa_{p^{\omega}}\right\} e^{2}\right]$, where [.] denotes the integral part and $\ln e=1$. The constant $c_{1}$ depends only on the Lipschitz character of the reference bodies $B_{m}$, $m=1, \ldots, M$.

We call the expressions (1.8 [1.9) the elastic Foldy-Lax approximation since the dominant terms are reminiscent to the exact form (called also the Foldy or the Foldy-Lax form) of the farfields derived in the scattering by finitely many point-like scatterers, see [15] for instance. These asymptotic expansions are useful for at least two reasons.

First, to estimate approximately the far-field, one needs only to compute the constant vectors $Q_{m}$ which are solutions of a linear algebraic system, i.e. (1.10). This reduces considerably the computational effort comparing it to the methods based on integral equations, for instance, especially for a large number of obstacles. If the number of obstacles is actually very large then these asymptotics suggest the kind of effective medium that can produce the same far-fields and provides the error rate between the fields generated by the obstacles and those generated by the effective medium.

Second, using formulas of the type (1.8) and (1.9), one can solve the inverse problems which consists of localizing the centers, $z_{m}$, of the obstacles from the far-field measurements using MUSIC type algorithm, for instance, and also estimating their sizes from the computed capacitances $C_{m}$.

As a first reference on this topic, we mention the book by P. Martin [19] where the multiple scattering issue is well discussed and documented in its different scales. When the obstacles are distributed periodically in the whole domain, then homogenization techniques apply, see for instance [9, 14, 18. As we see it in the previous theorem, we assume no periodicity. For such media, the type of result presented here are formally

\footnotetext{
${ }^{1}$ If, in particular, $\operatorname{diam}(\Omega) \max \left\{\kappa_{s} \omega, \kappa_{p} \omega\right\} e^{2}<1$, then $N_{\Omega}=1$ and hence $t=\left[\frac{1}{c_{p}^{2}}-4 \operatorname{diam}(\Omega)\left(\frac{\omega}{c_{s}^{3}}+\frac{\omega}{2 c_{p}^{3}}\right)\right]$. Assuming the Lamé coefficient $\lambda$ to be positive, then $c_{p}>c_{s}$. Hence, in this case, if $\Omega$ is such that $\operatorname{diam}(\Omega)<\frac{c_{s}}{\omega} \min \left\{\frac{1}{e^{2}}, \frac{c_{s}^{2}}{6 c_{p}^{2}}\right\}$ then $t>0$.
} 
derived, for the acoustic and electromagnetic models, in a series of works by A. Ramm, see [29 31] and the references therein for his recent related results, where he used the (rough) condition $\frac{a}{d} \ll 1$. Recently, in [11, we derived such approximation errors under a quite general condition on the denseness of the scatterers (i.e. involving $M$, a and $d$ ), i.e. of the form (1.7). The analysis is based on the use of integral equation methods and in particular the precise scaling of the surface layer potential operators. As it was mentioned in [11, the integral equation methods are also used in such a context, see for instance the series of works by $\mathrm{H}$. Ammari and H. Kang and their collaborators, as 3 and the references therein. The difference between their asymptotic expansion and the one described in the previous theorem is that their polarization tensors are build up from densities which are solutions of a system of integral equations while in the previous theorem the approximating terms are build up from the linear algebraic system (1.10). It is obvious that solving an algebraic system is less expansive than solving a system of integral equations, especially when dealing with many scatterers. In addition, due to motivations coming from inverse problems, apart from few works as [4, they consider well separated scatterers and hence their asymptotic expansions are given only in terms of the diameters $a$ of the scatterers. We should, however, emphasize that they provide asymptotic expansions at all the higher orders and they are valid also for extended scatterers. This opens door for many interesting applications, see 8 for instance.

Let us mention the variational approach by V. Maz'ya, A. Movchan 21] and by V. Maz'ya, A. Movchan and M. Nieves 24 where they study the Poisson problem and obtain estimates in forms similar to the previous theorem with weaker conditions of the form $\frac{a}{d} \leq c$, or $\frac{a}{d^{2}} \leq c$, (where, here $d$ is the smallest distance between the centers of the scatterers). In their analysis, they rely on the maximum principle to treat the boundary estimates. To avoid the use of the maximum principle, which is not valid due to the presence of the wave number $\kappa$, we use boundary integral equation methods. The price to pay is the need of the stronger assumption $\sqrt{M-1} \frac{a}{d} \leq c_{0}$. Another approach, based on the self-adjoint extensions of elliptic operator, is discussed in the works by S. A. Nazarov, and J. Sokolowski, see section 4 of [28] for instance, where they derive the asymptotic expansions for the Poisson problem. Let us finally, mention that the particular case where the obstacles have circular shapes has been considered recently by M. Cassier and C. Hazard in [10] for the scalar acoustic model.

Regarding the Lamé system, we cite the works [2,5,7] where, as we just mentioned, the asymptotics are given in terms of the size of the scatterers only. In these works, the authors considered transmission problems and showed that the corresponding moment tensors are in general anisotropic. If the inclusions are spherical, including the extreme cases of soft or rigid inclusions under certain conditions on the Lamé parameters, then these moment tensors are isotropic. Let us also mention the recent book 22], and the references therein, where an asymptotic expansion of the Green's tensor corresponding to the Dirichlet-Lamé problem (with zero frequency) in a bounded domain containing many small holes is derived.

The goal of the present work is to extend the results in [11] to the Lamé system and derive the error of the approximation explicitly in terms of the whole denseness of the scatterers, i.e. $M, a$ and $d$. To our best knowledge this is the first result with this generality for the Lamé system, compare to [3] and [22]. The analysis is based on the use of the integral equation methods and the different scaling of the corresponding boundary integral operators. Due to the coupling of the two fundamental waves, i.e. the P-waves and the S-waves, at the boundaries of the obstacles, the analysis cannot be reduced to the one of our previous work [1]. Indeed, a considerable work is needed to derive explicitly these scaling, characterize the dominant parts of the elastic fields and justify the invertibility of corresponding algebraic system (1.10).

Before concluding the introduction, we state the following corollary where more precise estimates than those given in Theorem 1.2 are presented under some additional conditions on the scatterers.

Corollary 1.3. Assume that the conditions of Theorem 1.2 are fulfilled.

1. We assume, in addition, that $D_{m}$ are balls with the same diameter a for $m=1, \ldots, M$, then we have the following asymptotic expansion for the P-part, $U_{p}^{\infty}(\hat{x}, \theta)$, and the $S$-part, $U_{s}^{\infty}(\hat{x}, \theta)$, of the far-field pattern:

$$
U_{p}^{\infty}(\hat{x}, \theta)=\frac{1}{4 \pi c_{p}^{2}}(\hat{x} \otimes \hat{x})\left[\sum_{m=1}^{M} e^{-i \frac{\omega}{c_{p}} \hat{x} \cdot z_{m}} Q_{m}\right.
$$




$$
\begin{aligned}
& \left.+O\left(M\left[a^{2}+\frac{a^{3}}{d^{5-3 \alpha}}+\frac{a^{4}}{d^{9-6 \alpha}}\right]+M(M-1)\left[\frac{a^{3}}{d^{2 \alpha}}+\frac{a^{4}}{d^{4-\alpha}}+\frac{a^{4}}{d^{5-2 \alpha}}\right]+M(M-1)^{2} \frac{a^{4}}{d^{3 \alpha}}\right)\right], \\
& U_{s}^{\infty}(\hat{x}, \theta)=\frac{1}{4 \pi c_{s}^{2}}(I-\hat{x} \otimes \hat{x})\left[\sum_{m=1}^{M} e^{-i \frac{\omega}{c_{s}} \hat{x} \cdot z_{m}} Q_{m}\right. \\
& \left.+O\left(M\left[a^{2}+\frac{a^{3}}{d^{5-3 \alpha}}+\frac{a^{4}}{d^{9-6 \alpha}}\right]+M(M-1)\left[\frac{a^{3}}{d^{2 \alpha}}+\frac{a^{4}}{d^{4-\alpha}}+\frac{a^{4}}{d^{5-2 \alpha}}\right]+M(M-1)^{2} \frac{a^{4}}{d^{3 \alpha}}\right)\right]
\end{aligned}
$$

where $0<\alpha \leq 1$.

Consider now the special case $d=a^{t}, M=a^{-s}$ with $t, s>0$. Then the asymptotic expansions 1.13. 1.14) can be rewritten as

$$
\begin{aligned}
U_{p}^{\infty}(\hat{x}, \theta)= & \frac{1}{4 \pi c_{p}^{2}}(\hat{x} \otimes \hat{x})\left[\sum_{m=1}^{M} e^{-i \frac{\omega}{c_{p}} \hat{x} \cdot z_{m}} Q_{m}\right. \\
& \left.+O\left(a^{2-s}+a^{3-s-5 t+3 t \alpha}+a^{4-s-9 t+6 t \alpha}+a^{3-2 s-2 t \alpha}+a^{4-3 s-3 t \alpha}+a^{4-2 s-5 t+2 t \alpha}\right)\right], \\
U_{s}^{\infty}(\hat{x}, \theta)= & \frac{1}{4 \pi c_{s}^{2}}(I-\hat{x} \otimes \hat{x})\left[\sum_{m=1}^{M} e^{-i \frac{\omega}{c_{s}} \hat{x} \cdot z_{m}} Q_{m}\right. \\
& \left.+O\left(a^{2-s}+a^{3-s-5 t+3 t \alpha}+a^{4-s-9 t+6 t \alpha}+a^{3-2 s-2 t \alpha}+a^{4-3 s-3 t \alpha}+a^{4-2 s-5 t+2 t \alpha}\right)\right] .
\end{aligned}
$$

As the diameter a tends to zero the error term tends to zero for $t$ and $s$ such that $0<t<1$ and $0<s<\min \left\{2(1-t), \frac{7-5 t}{4}, \frac{12-9 t}{7}, \frac{20-15 t}{12}, \frac{4}{3}-t \alpha\right\}$. In particular for $t=\frac{1}{3}, s=1$, we have

$$
\begin{aligned}
U_{p}^{\infty}(\hat{x}, \theta) & =\frac{1}{4 \pi c_{p}^{2}}(\hat{x} \otimes \hat{x})\left[\sum_{m=1}^{M} e^{-i \frac{\omega}{c_{p}} \hat{x} \cdot z_{m}} Q_{m}+O\left(a+a^{2 \alpha}+a^{1-\alpha}+a^{\frac{1+2 \alpha}{3}}\right)\right] \\
& =\frac{1}{4 \pi c_{p}^{2}}(\hat{x} \otimes \hat{x})\left[\sum_{m=1}^{M} e^{-i \frac{\omega}{c_{p}} \hat{x} \cdot z_{m}} Q_{m}+O\left(a^{\frac{1}{2}}\right)\right], \quad\left[\text { obtained for } \alpha=\frac{1}{4}\right], \\
U_{s}^{\infty}(\hat{x}, \theta) & =\frac{1}{4 \pi c_{s}^{2}}(I-\hat{x} \otimes \hat{x})\left[\sum_{m=1}^{M} e^{-i \frac{\omega}{c_{s}} \hat{x} \cdot z_{m}} Q_{m}+O\left(a+a^{2 \alpha}+a^{1-\alpha}+a^{\frac{1+2 \alpha}{3}}\right)\right] \\
& =\frac{1}{4 \pi c_{s}^{2}}(I-\hat{x} \otimes \hat{x})\left[\sum_{m=1}^{M} e^{-i \frac{\omega}{c_{s}} \hat{x} \cdot z_{m}} Q_{m}+O\left(a^{\frac{1}{2}}\right)\right], \quad\left[\text { obtained for } \alpha=\frac{1}{4}\right] .
\end{aligned}
$$

2. Actually, the results (1.13 1.14) and (1.15 1.16) are valid for the non-flat Lipschitz obstacles $D_{m}=$ $\epsilon B_{m}+z_{m}, m=1, \ldots, M$ with the same diameter a, i.e. $D_{m}$ 's are Lipschitz obstacles and there exist constants $t_{m} \in(0,1]$ such that

$$
B_{t_{m} \frac{a}{2}}^{3}\left(z_{m}\right) \subset D_{m} \subset B_{\frac{a}{2}}^{3}\left(z_{m}\right),
$$

where $t_{m}$ are assumed to be uniformly bounded from below by a positive constant.

The results of this corollary can be used to derive the effective medium by perforation using many small bodies. In addition, (1.15) and (1.16) ensure the rate of the error in deriving such an effective medium. Details on this topic will be reported in a future work.

The rest of the paper is organized as follows. In section 2, we give the proof of the asymptotic expansion (1.8 (1.9). In section 3, we study the solvability of the linear algebraic system (1.10). Finally, in section 4, as an appendix, we derive some needed properties of the layer potentials. 


\section{Proof of Theorem 1.2}

We wish to warm the reader that in our analysis we use sometimes the parameter $\epsilon$ and some other times the parameter $a$ as they appear naturally in the estimates. But we bear in mind the relation (1.5) between $a$ and $\epsilon$.

\subsection{The fundamental solution}

The Kupradze matrix $\Gamma^{\omega}=\left(\Gamma_{i j}^{\omega}\right)_{i, j=1}^{3}$ of the fundamental solution to the Navier equation is given by

$$
\Gamma^{\omega}(x, y)=\frac{1}{\mu} \Phi_{\kappa_{s} \omega}(x, y) \mathbf{I}+\frac{1}{\omega^{2}} \nabla_{\mathrm{x}} \nabla_{\mathrm{x}}^{\top}\left[\Phi_{\kappa_{\mathrm{s}} \omega}(\mathrm{x}, \mathrm{y})-\Phi_{\kappa_{\mathrm{p}} \omega}(\mathrm{x}, \mathrm{y})\right]
$$

where $\Phi_{\kappa}(x, y)=\frac{1}{4 \pi} \exp (i \kappa|x-y|)$ denotes the free space fundamental solution of the Helmholtz equation $\left(\Delta+\kappa^{2}\right) u=0$ in $\mathbb{R}^{3}$. The asymptotic behavior of Kupradze tensor at infinity is given as follows

$$
\Gamma^{\omega}(x, y)=\frac{1}{4 \pi c_{p}^{2}} \hat{x} \otimes \hat{x} \frac{e^{i \kappa_{p} \omega|x|}}{|x|} e^{-i \kappa_{p} \omega \hat{x} \cdot y}+\frac{1}{4 \pi c_{s}^{2}}(I-\hat{x} \otimes \hat{x}) \frac{e^{i \kappa_{s} \omega|x|}}{|x|} e^{-i \kappa_{s} \omega \hat{x} \cdot y}+O\left(|x|^{-2}\right)
$$

with $\hat{x}=\frac{x}{|x|} \in \mathbb{S}^{2}$ and $I$ being the identity matrix in $\mathbb{R}^{3}$, see [1] for instance. As mentioned in [6], (2.1) can also be represented as

$$
\begin{aligned}
\Gamma^{\omega}(x, y)= & \frac{1}{4 \pi} \sum_{l=0}^{\infty} \frac{i^{l}}{l !(l+2)} \frac{1}{\omega^{2}}\left((l+1) \kappa_{s^{\omega}}^{l+2}+\kappa_{p^{\omega}}^{l+2}\right)|x-y|^{l-1} \mathbf{I} \\
& -\frac{1}{4 \pi} \sum_{l=0}^{\infty} \frac{i^{l}}{l !(l+2)} \frac{(l-1)}{\omega^{2}}\left(\kappa_{s^{\omega}}^{l+2}-\kappa_{p^{\omega}}^{l+2}\right)|x-y|^{l-3}(x-y) \otimes(x-y),
\end{aligned}
$$

from which we can get the gradient

$$
\begin{aligned}
\nabla_{y} \Gamma^{\omega}(x, y)= & -\frac{1}{4 \pi} \sum_{l=0}^{\infty} \frac{i^{l}}{l !(l+2)} \frac{(l-1)}{\omega^{2}}\left[\left((l+1) \kappa_{s^{\omega}}^{l+2}+\kappa_{p^{\omega}}^{l+2}\right)|x-y|^{l-3}(x-y) \otimes \mathbf{I}\right. \\
& \left.-\left(\kappa_{s^{\omega}}^{l+2}-\kappa_{p^{\omega}}^{l+2}\right)|x-y|^{l-3}\left((l-3)|x-y|^{-2} \otimes^{3}(x-y)+\mathbf{I} \otimes(\mathrm{x}-\mathrm{y})+(\mathrm{x}-\mathrm{y}) \otimes \mathbf{I}\right)\right]
\end{aligned}
$$

\subsection{The representation via double layer potential}

We start with the following proposition on the solution of the problem (1.1]1.3) via the method of integral equations.

Proposition 2.1. There exists $a_{0}>0$, such that if $a<a_{0} 2$ the solution of the problem (1.1] 1.3 ) is of the form

$$
U^{t}(x)=U^{i}(x)+\sum_{m=1}^{M} \int_{\partial D_{m}} \frac{\partial \Gamma^{\omega}(x, s)}{\partial \nu_{m}(s)} \sigma_{m}(s) d s, x \in \mathbb{R}^{3} \backslash\left(\bigcup_{m=1}^{M} \bar{D}_{m}\right),
$$

where $\sigma_{m} \in H^{r}\left(\partial D_{m}\right)$, with $r \in[0,1]$, for $m=1,2, \ldots, M$, and $\frac{\partial}{\partial \nu_{m}}(\cdot)$ denotes the co-normal derivative on $\partial D_{m}$ and is defined as

$$
\frac{\partial}{\partial \nu_{m}}(\cdot):=\lambda(\operatorname{div} \cdot) N_{m}+\mu\left(\nabla \cdot+\nabla \cdot \cdot^{\top}\right) N_{m} \quad \text { on } \partial D_{m}
$$

with $N_{m}$ the outward unit normal vector of $\partial D_{m}$.

\footnotetext{
${ }^{2}$ The condition on $a$ can be replaced by a condition on $\omega$ as it can be seen from the proof.
} 
Proof. of Proposition 2.1. We look for the solution of the problem (1.1) 1.3) of the form (2.5), then from the Dirichlet boundary condition (1.2) and the jumps of the double layer potentials, we obtain

$$
\frac{\sigma_{j}\left(s_{j}\right)}{2}+\int_{\partial D_{j}} \frac{\partial \Gamma^{\omega}\left(s_{j}, s\right)}{\partial \nu_{j}(s)} \sigma_{j}(s) d s+\sum_{\substack{m=1 \\ m \neq j}}^{M} \int_{\partial D_{m}} \frac{\partial \Gamma^{\omega}\left(s_{j}, s\right)}{\partial \nu_{m}(s)} \sigma_{m}(s) d s=-U^{i}\left(s_{j}\right), \forall s_{j} \in \partial D_{j}, j=1, \ldots, M
$$

One can write the system (2.7) in a compact form as $\left(\frac{1}{2} \mathbf{I}+D L+D K\right) \sigma=-U^{I n}$ with $\mathbf{I}:=\left(\mathbf{I}_{m j}\right)_{m, j=1}^{M}$, $D L:=\left(D L_{m j}\right)_{m, j=1}^{M}$ and $D K:=\left(D K_{m j}\right)_{m, j=1}^{M}$, where

$$
\begin{array}{r}
\mathbf{I}_{m j}=\left\{\begin{array}{l}
I, \text { Identity operator } \begin{array}{r}
m=j \\
\text { 0, zero operator }
\end{array} \text { else }
\end{array}, \quad D L_{m j}=\left\{\begin{array}{cc}
\mathcal{D}_{m j} & m=j \\
0 & \text { else }
\end{array}, \quad D K\right.\right. \\
U^{I n}=U^{I n}\left(s_{1}, \ldots, s_{M}\right):=\left(U^{i}\left(s_{1}\right), \ldots, U^{i}\left(s_{M}\right)\right)^{T} \\
\text { and } \sigma=\sigma\left(s_{1}, \ldots, s_{M}\right):=\left(\sigma_{1}\left(s_{1}\right), \ldots, \sigma_{M}\left(s_{M}\right)\right)^{T} .
\end{array}
$$

Here, for the indices $m$ and $j$ fixed, $\mathcal{D}_{m j}$ is the integral operator

$$
\mathcal{D}_{m j}\left(\sigma_{j}\right)(t):=\int_{\partial D_{j}} \frac{\partial \Gamma^{\omega}(t, s)}{\partial \nu_{j}(s)} \sigma_{j}(s) d s .
$$

The operator $\frac{1}{2} I+\mathcal{D}_{m m}: H^{r}\left(\partial D_{m}\right) \rightarrow H^{r}\left(\partial D_{m}\right)$ is Fredholm with zero index and for $m \neq j, \mathcal{D}_{m j}:$ $H^{r}\left(\partial D_{j}\right) \rightarrow H^{r}\left(\partial D_{m}\right)$ is compact for $0 \leq r \leq 1$, when $\partial D_{m}$ has a Lipschitz regularity, see [20, 26, 27, 3 So, $\left(\frac{1}{2} \mathbf{I}+D L+D K\right): \prod_{m=1}^{M} H^{r}\left(\partial D_{m}\right) \rightarrow \prod_{m=1}^{M} H^{r}\left(\partial D_{m}\right)$ is Fredholm with zero index. We induce the product of spaces by the maximum of the norms of the space. To show that $\left(\frac{1}{2} \mathbf{I}+D L+D K\right)$ is invertible it is enough to show that it is injective. i.e. $\left(\frac{1}{2} \mathbf{I}+D L+D K\right) \sigma=0$ implies $\sigma=0$.

Write,

$$
\tilde{U}(x)=\sum_{m=1}^{M} \int_{\partial D_{m}} \frac{\partial \Gamma^{\omega}\left(s_{j}, s\right)}{\partial \nu_{m}(s)} \sigma_{m}(s) d s, \text { in } \mathbb{R}^{3} \backslash\left(\bigcup_{m=1}^{M} \bar{D}_{m}\right)
$$

and

$$
\tilde{\tilde{U}}(x)=\sum_{m=1}^{M} \int_{\partial D_{m}} \frac{\partial \Gamma^{\omega}\left(s_{j}, s\right)}{\partial \nu_{m}(s)} \sigma_{m}(s) d s, \text { in } \bigcup_{m=1}^{M} D_{m} .
$$

Then $\tilde{U}$ satisfies $\Delta^{e} \tilde{U}+\omega^{2} \tilde{U}=0$ for $x \in \mathbb{R}^{3} \backslash\left(\bigcup_{m=1}^{M} \bar{D}_{m}\right)$, with K.R.C and $\tilde{U}(x)=0$ on $\bigcup_{m=1}^{M} \partial D_{m}$. Similarly, $\tilde{\tilde{U}}$ satisfies $\Delta^{e} \tilde{\tilde{U}}+\omega^{2} \tilde{\tilde{U}}=0$ for $x \in \bigcup_{m=1}^{M} D_{m}$ with $\tilde{\tilde{U}}(x)=0$ on $\bigcup_{m=1}^{M} \partial D_{m}$. Taking the trace on $\partial D_{m}$, $m=1, \ldots, M$,

$$
\tilde{U}(s)=0 \Longrightarrow \mathcal{D}_{m m}\left(\sigma_{m}\right)(s)+\frac{\sigma_{m}(s)}{2}+\sum_{j \neq m} \mathcal{D}_{m j}\left(\sigma_{j}\right)(s)=0
$$

and

$$
\tilde{\tilde{U}}(s)=0 \Longrightarrow \mathcal{D}_{m m}\left(\sigma_{m}\right)(s)-\frac{\sigma_{m}(s)}{2}+\sum_{j \neq m} \mathcal{D}_{m j}\left(\sigma_{j}\right)(s)=0
$$

\footnotetext{
${ }^{3}$ In [20 26, 27], this property is proved for the case $\omega=0$. By a perturbation argument, we have the same results for every $\omega$ in $\left[0, \omega_{\max }\right]$, assuming that $\omega_{\max }$ is smaller than the first eigenvalue $w_{e l}$ of the Dirichlet-Lamé operator in $D_{m}$. By a comparison theorem, see [22] (6.131) in Lemma 6.3.6] for instance, we know that $\mu w_{L} \leq w_{e l}$ where $w_{L}$ is the first eigenvalue of the Dirichlet-Laplacian operator in $D_{m}$. Now, we know that $\left(\frac{1}{a} \sqrt[3]{\frac{4 \pi}{3}} \mathrm{j}_{1 / 2,1}\right)^{2} \leq w_{L}$. Then, we need $\omega_{\max }<\frac{\sqrt{\mu}}{a} \sqrt[3]{\frac{4 \pi}{3}} \mathrm{j}_{1 / 2,1}$ which is satisfied if $a<\frac{\sqrt{\mu}}{\omega_{\max }} \sqrt[3]{\frac{4 \pi}{3}} \mathrm{j}_{1 / 2,1}:=a_{0}$. Here $\mathrm{j}_{1 / 2,1}$ is the 1st positive zero of the Bessel function $\mathrm{J}_{1 / 2}$.
} 
for $s \in \partial D_{m}$ and for $m=1, \ldots, M$. Difference between (2.12) and (2.13) implies that, $\sigma_{m}=0$ for all $m$. 8 We conclude then that $\frac{1}{2} \mathbf{I}+D L+D K:=\frac{1}{2} \mathbf{I}+\mathcal{D}: \prod_{m=1}^{M} H^{r}\left(\partial D_{m}\right) \rightarrow \prod_{m=1}^{M} H^{r}\left(\partial D_{m}\right)$ is invertible.

\subsection{An appropriate estimate of the densities $\sigma_{m}, m=1, \ldots, M$}

From the above theorem, we have the following representation of $\sigma$ :

$$
\begin{aligned}
\sigma & =\left(\frac{1}{2} \mathbf{I}+D L+D K\right)^{-1} U^{I n} \\
& =\left(\frac{1}{2} \mathbf{I}+D L\right)^{-1}\left(\mathbf{I}+\left(\frac{1}{2} \mathbf{I}+D L\right)^{-1} D K\right)^{-1} U^{I n} \\
& =\left(\frac{1}{2} \mathbf{I}+D L\right)^{-1} \sum_{l=0}^{\infty}\left(-\left(\frac{1}{2} \mathbf{I}+D L\right)^{-1} D K\right)^{l} U^{I n}, \quad \text { if }\left\|\left(\frac{1}{2} \mathbf{I}+D L\right)^{-1} D K\right\|<1
\end{aligned}
$$

The operator $\frac{1}{2} \mathbf{I}+D L$ is invertible since it is Fredholm of index zero and injective. This implies that

$$
\|\sigma\| \leq \frac{\left\|\left(\frac{1}{2} \mathbf{I}+D L\right)^{-1}\right\|}{1-\left\|\left(\frac{1}{2} \mathbf{I}+D L\right)^{-1}\right\|\|D K\|}\left\|U^{I n}\right\|
$$

Here,

$$
\begin{aligned}
& \|D K\|:=\|D K\|_{\mathcal{L}\left(\prod_{m=1}^{M} L^{2}\left(\partial D_{m}\right), \prod_{m=1}^{M} L^{2}\left(\partial D_{m}\right)\right)} \\
& \equiv \max _{m=1}^{M} \sum_{j=1}^{M}\left\|D K_{m j}\right\|_{\mathcal{L}\left(L^{2}\left(\partial D_{j}\right), L^{2}\left(\partial D_{m}\right)\right)} \\
& =\max _{m=1}^{M} \sum_{\substack{j=1 \\
j \neq m}}^{M}\left\|\mathcal{D}_{m j}\right\|_{\mathcal{L}\left(L^{2}\left(\partial D_{j}\right), L^{2}\left(\partial D_{m}\right)\right)}, \\
& \left.\left\|\left(\frac{1}{2} \mathbf{I}+D L\right)^{-1}\right\|:=\left\|\left(\frac{1}{2} \mathbf{I}+D L\right)^{-1}\right\|_{\mathcal{L}\left(\prod_{m=1}^{M} L^{2}\left(\partial D_{m}\right), \prod_{m=1}^{M} L^{2}\left(\partial D_{m}\right)\right.}\right) \\
& \equiv \max _{m=1}^{M} \sum_{j=1}^{M}\left\|\left(\frac{1}{2} \mathbf{I}+D L\right)_{m j}^{-1}\right\|_{\mathcal{L}\left(L^{2}\left(\partial D_{m}\right), L^{2}\left(\partial D_{j}\right)\right)} \\
& =\max _{m=1}^{M}\left\|\left(\frac{1}{2} \mathbf{I}+\mathcal{D}_{m m}\right)^{-1}\right\|_{\mathcal{L}\left(L^{2}\left(\partial D_{m}\right), L^{2}\left(\partial D_{m}\right)\right)},
\end{aligned}
$$

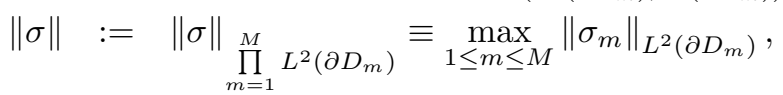

$$
\begin{aligned}
& \text { and } \\
& \left\|U^{I n}\right\|:=\left\|U^{I n}\right\|_{\prod_{m=1}^{M} L^{2}\left(\partial D_{m}\right)} \equiv \max _{1 \leq m \leq M}\left\|U^{i}\right\|_{L^{2}\left(\partial D_{m}\right)} .
\end{aligned}
$$

In the following proposition, we provide conditions under which $\left\|\left(\frac{1}{2} \mathbf{I}+D L\right)^{-1}\right\|\|D K\|<1$ and then estimate $\|\sigma\|$ via (2.15).

Proposition 2.2. There exists a constant c̀ depending only on the size of $\Omega$, the Lipschitz character of $B_{m}, m=1, \ldots, M, d_{\max }$, and $\omega_{\max }$ such that if

$$
\sqrt{M-1} \epsilon<\grave{c} d, \quad \text { then } \quad\left\|\sigma_{m}\right\|_{L^{2}\left(\partial D_{m}\right)} \leq c \epsilon
$$

where $c$ is a positive constant depending only on the Lipschitz character of $B_{m}$. 
Proof of Proposition 2.2.

For any functions $f, g$ defined on $\partial D_{\epsilon}$ and $\partial B$ respectively, we define

$$
(f)^{\wedge}(\xi):=\hat{f}(\xi):=f(\epsilon \xi+z) \quad \text { and } \quad(g)^{\vee}(x):=\check{g}(x):=g\left(\frac{x-z}{\epsilon}\right) .
$$

Let $T_{1}$ and $T_{2}$ be an orthonormal basis for the tangent plane to $\partial D_{\epsilon}$ at $\mathrm{x}$ and let $\partial / \partial T=\sum_{l=1}^{2} \partial / \partial T_{p} T_{p}$, denote the tangential derivative on $\partial D_{\epsilon}$. Then the space $H^{1}\left(\partial D_{\epsilon}\right)$ is defined as

$$
H^{1}\left(\partial D_{\epsilon}\right):=\left\{\phi \in L^{2}\left(\partial D_{\epsilon}\right) ; \partial \phi / \partial T \in L^{2}\left(\partial D_{\epsilon}\right)\right\} .
$$

We have the following lemma from [1].

Lemma 2.3. Suppose $0<\epsilon \leq 1$ and $D_{\epsilon}:=\epsilon B+z \subset \mathbb{R}^{n}$. Then for every $\psi \in L^{2}\left(\partial D_{\epsilon}\right)$ and $\phi \in H^{1}\left(\partial D_{\epsilon}\right)$, we have

$$
\|\psi\|_{L^{2}\left(\partial D_{\epsilon}\right)}=\epsilon^{\frac{n-1}{2}}\|\hat{\psi}\|_{L^{2}(\partial B)}
$$

and

$$
\epsilon^{\frac{n-1}{2}}\|\hat{\phi}\|_{H^{1}(\partial B)} \leq\|\phi\|_{H^{1}\left(\partial D_{\epsilon}\right)} \leq \epsilon^{\frac{n-3}{2}}\|\hat{\phi}\|_{H^{1}(\partial B)} .
$$

We divide the rest of the proof of Proposition 2.2 into two steps. In the first step, we assume we have a single obstacle and then in the second step we deal with the multiple obstacle case.

\subsubsection{The case of a single obstacle}

Let us consider a single obstacle $D_{\epsilon}:=\epsilon B+z$. Then define the operator $\mathcal{D}_{D_{\epsilon}}: L^{2}\left(\partial D_{\epsilon}\right) \rightarrow L^{2}\left(\partial D_{\epsilon}\right)$ by

$$
\left(\mathcal{D}_{D_{\epsilon}} \psi\right)(s)=\int_{\partial D_{\epsilon}} \frac{\partial \Gamma^{\omega}(s, t)}{\partial \nu(t)} \psi(t) d t
$$

Following the arguments in the proof of Proposition 2.1 the integral operator $\frac{1}{2} I+\mathcal{D}_{D_{\epsilon}}: L^{2}\left(\partial D_{\epsilon}\right) \rightarrow L^{2}\left(\partial D_{\epsilon}\right)$ is invertible. If we consider the problem (1.1]1.3) in $\mathbb{R}^{3} \backslash \bar{D}_{\epsilon}$, we obtain

$$
\sigma=\left(\frac{1}{2} I+\mathcal{D}_{D_{\epsilon}}\right)^{-1} U^{i}, \text { where } D L+D K=: \mathcal{D}_{D_{\epsilon}}
$$

and then

$$
\|\sigma\|_{L^{2}\left(\partial D_{\epsilon}\right)} \leq\left\|\left(\frac{1}{2} I+\mathcal{D}_{D_{\epsilon}}\right)^{-1}\right\|_{\mathcal{L}\left(L^{2}\left(\partial D_{\epsilon}\right), L^{2}\left(\partial D_{\epsilon}\right)\right)}\left\|U^{i}\right\|_{L^{2}\left(\partial D_{\epsilon}\right)} .
$$

Lemma 2.4. Let $\phi, \psi \in L^{2}\left(\partial D_{\epsilon}\right)$. Then,

$$
\begin{gathered}
\mathcal{D}_{D_{\epsilon}} \psi=\left(\mathcal{D}_{B}^{\epsilon} \hat{\psi}\right)^{\vee}, \\
\left(\frac{1}{2} I+\mathcal{D}_{D_{\epsilon}}\right) \psi=\left(\left(\frac{1}{2} I+\mathcal{D}_{B}^{\epsilon}\right) \hat{\psi}\right)^{\vee}, \\
\left(\frac{1}{2} I+\mathcal{D}_{D_{\epsilon}}\right)^{-1} \phi=\left(\left(\frac{1}{2} I+\mathcal{D}_{B}^{\epsilon}\right)^{-1} \hat{\phi}\right)^{\vee} \\
\left\|\left(\frac{1}{2} I+\mathcal{D}_{D_{\epsilon}}\right)^{-1}\right\|_{\mathcal{L}\left(L^{2}\left(\partial D_{\epsilon}\right), L^{2}\left(\partial D_{\epsilon}\right)\right)}=\left\|\left(\frac{1}{2} I+\mathcal{D}_{B}^{\epsilon}\right)^{-1}\right\|_{\mathcal{L}\left(L^{2}(\partial B), L^{2}(\partial B)\right)}
\end{gathered}
$$

and

$$
\left\|\left(\frac{1}{2} I+\mathcal{D}_{D_{\epsilon}}\right)^{-1}\right\|_{\mathcal{L}\left(H^{1}\left(\partial D_{\epsilon}\right), H^{1}\left(\partial D_{\epsilon}\right)\right)} \leq \epsilon^{-1}\left\|\left(\frac{1}{2} I+\mathcal{D}_{B}^{\epsilon}\right)^{-1}\right\|_{\mathcal{L}\left(H^{1}(\partial B), H^{1}(\partial B)\right)}
$$

with $\mathcal{D}_{B}^{\epsilon} \hat{\psi}(\xi):=\int_{\partial B} \frac{\partial \Gamma^{\epsilon \omega}(\xi, \eta)}{\partial \nu(\eta)} \hat{\psi}(\eta) d \eta$. 
- We have,

$$
\begin{aligned}
\mathcal{D}_{D_{\epsilon}} \psi(s) & =\int_{\partial D_{\epsilon}} \frac{\partial \Gamma^{\omega}(s, t)}{\partial \nu(t)} \psi(t) d t \\
& \left.=\int_{\partial D_{\epsilon}}\left[\lambda\left(\operatorname{div}_{t} \Gamma^{\omega}(s, t)\right) N_{t}+\mu\left(\nabla_{t} \Gamma^{\omega}(s, t)+\left(\nabla_{t} \Gamma^{\omega}(s, t)\right)^{\top}\right) N_{t}\right)\right] \psi(t) d t \\
& =\int_{\partial B} \epsilon^{-2}\left[\lambda\left(\operatorname{div}_{\eta} \Gamma^{\epsilon \omega}(\xi, \eta)\right) N_{\eta}+\mu\left(\nabla_{\eta} \Gamma^{\epsilon \omega}(\xi, \eta)+\left(\nabla_{\eta} \Gamma^{\epsilon \omega}(\xi, \eta)\right)^{\top}\right) N_{\eta}\right] \psi(\epsilon \eta+z) \epsilon^{2} d \eta \\
& =\int_{\partial B} \frac{\partial \Gamma^{\epsilon \omega}(\xi, \eta)}{\partial \nu(\eta)} \psi(\epsilon \eta+z) d \eta \\
& =\mathcal{D}_{B}^{\epsilon} \hat{\psi}(\xi) .
\end{aligned}
$$

The above gives us (2.26). From (2.26), we can obtain (2.27).

- The following equalities

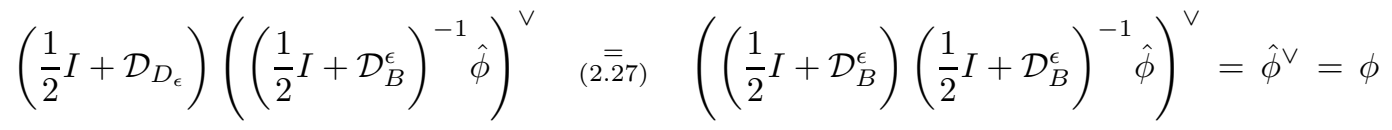

provide us (2.28).

- The following equalities

$$
\begin{aligned}
& \left\|\left(\frac{1}{2} I+\mathcal{D}_{D_{\epsilon}}\right)^{-1}\right\|_{\mathcal{L}\left(L^{2}\left(\partial D_{\epsilon}\right), L^{2}\left(\partial D_{\epsilon}\right)\right)} \quad:=\quad \operatorname{Sup}_{\phi(\neq 0) \in L^{2}\left(\partial D_{\epsilon}\right)} \frac{\left\|\left(\frac{1}{2} I+\mathcal{D}_{D_{\epsilon}}\right)^{-1} \phi\right\|_{L^{2}\left(\partial D_{\epsilon}\right)}}{\|\phi\|_{L^{2}\left(\partial D_{\epsilon}\right)}}
\end{aligned}
$$



$$
\begin{aligned}
& \underset{\operatorname{Sup}_{(2.28)}}{\overline{\overline{2}}} \quad \frac{\left\|\left(\frac{1}{2} I+\mathcal{D}_{B}^{\epsilon}\right)^{-1} \hat{\phi}\right\|_{L^{2}(\partial B)}}{\|\hat{\phi}\|_{L^{2}(\partial B)}} \\
& =\left\|\left(\frac{1}{2} I+\mathcal{D}_{B}^{\epsilon}\right)^{-1}\right\|_{\mathcal{L}\left(L^{2}(\partial B), L^{2}(\partial B)\right)}
\end{aligned}
$$

provide us (2.29). By proceeding in the similar manner we can obtain (2.30) as mentioned below,

$$
\begin{aligned}
& \left\|\left(\frac{1}{2} I+\mathcal{D}_{D_{\epsilon}}\right)^{-1}\right\|_{\mathcal{L}\left(H^{1}\left(\partial D_{\epsilon}\right), H^{1}\left(\partial D_{\epsilon}\right)\right)} \quad:=\operatorname{Sup}_{\substack{\operatorname{Sup}^{1}(\neq 0) \in H^{1}\left(\partial D_{\epsilon}\right) \\
\left\|\left(\frac{1}{2} I+\mathcal{D}_{D_{\epsilon}}\right)^{-1} \phi\right\|_{H^{1}\left(\partial D_{\epsilon}\right)}}}
\end{aligned}
$$

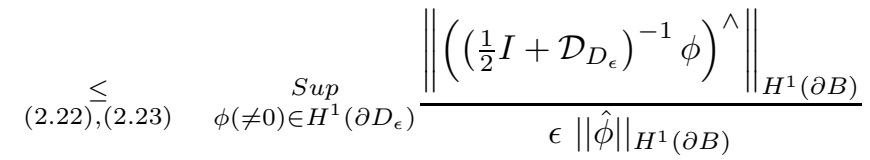

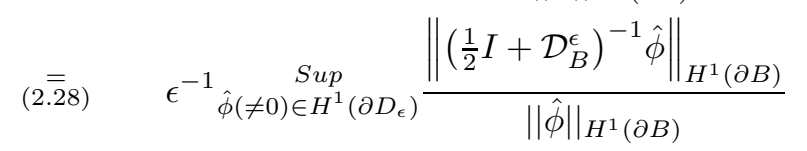




$$
=\quad \epsilon^{-1}\left\|\left(\frac{1}{2} I+\mathcal{D}_{B}^{\epsilon}\right)^{-1}\right\|_{\mathcal{L}\left(H^{1}(\partial B), H^{1}(\partial B)\right)}
$$

The next lemma provides us with an estimate of the left hand side of (2.29) by a constant $C$ with a useful dependence of $C$ in terms of $B$ through its Lipschitz character and $\omega$.

Lemma 2.5. The operator norm of $\left(\frac{1}{2} I+\mathcal{D}_{D_{\epsilon}}\right)^{-1}: L^{2}\left(\partial D_{\epsilon}\right) \rightarrow L^{2}\left(\partial D_{\epsilon}\right)$ satisfies the estimate

$$
\left\|\left(\frac{1}{2} I+\mathcal{D}_{D_{\epsilon}}\right)^{-1}\right\|_{\mathcal{L}\left(L^{2}\left(\partial D_{\epsilon}\right), L^{2}\left(\partial D_{\epsilon}\right)\right)} \leq \grave{C}_{6}
$$

with $\grave{C}_{6}:=\frac{4 \pi\left\|\left(\frac{1}{2} I+\mathcal{D}_{B}^{i_{\omega}}\right)^{-1}\right\|_{\mathcal{L}\left(L^{2}(\partial B), L^{2}(\partial B)\right)}}{4 \pi-\left[\frac{4 \lambda+17 \mu}{2 c_{s}^{4}}+\frac{12 \lambda+9 \mu}{2 c_{p}^{4}}\right] \omega^{2} \epsilon^{2}|\partial B|\left\|\left(\frac{1}{2} I+\mathcal{D}_{B}^{i_{\omega}}\right)^{-1}\right\|_{\mathcal{L}\left(L^{2}(\partial B), L^{2}(\partial B)\right)}}$. Here, $\mathcal{D}_{B}^{i_{\omega}}: L^{2}(\partial B) \rightarrow L^{2}(\partial B)$ is the double layer potential with the zero frequency.

Here we should mention that if $\epsilon^{2} \leq \frac{\pi}{\left[\frac{4 \lambda+17 \mu}{2 c_{s}^{4}}+\frac{12 \lambda+9 \mu}{2 c_{p}^{4}}\right] \omega^{2}|\partial B|\left\|\left(\frac{1}{2} I+\mathcal{D}_{B}^{i \omega}\right)^{-1}\right\|_{\mathcal{L}\left(L^{2}(\partial B), L^{2}(\partial B)\right)}}$, then $\grave{C}_{6}$ is bounded by $\frac{4}{3}\left\|\left(\frac{1}{2} I+\mathcal{D}_{B}^{i_{\omega}}\right)^{-1}\right\|_{\mathcal{L}\left(L^{2}(\partial B), L^{2}(\partial B)\right)}$, which is a universal constant depending only on $\partial B$ through its Lipschitz character.

Proof. of Lemma 2.5. To estimate the operator norm of $\left(\frac{1}{2} I+\mathcal{D}_{D_{\epsilon}}\right)^{-1}$ we decompose $\mathcal{D}_{D_{\epsilon}}=: \mathcal{D}_{D_{\epsilon}}^{\omega}=$ $\mathcal{D}_{D_{\epsilon}}^{i_{\omega}}+\mathcal{D}_{D_{\epsilon}}^{d_{\omega}}$ into two parts $\mathcal{D}_{D_{\epsilon}}^{i_{\omega}}$ (independent of $\omega$ ) and $\mathcal{D}_{D_{\epsilon}}^{d_{\omega}}$ ( dependent of $\omega$ ) given by

$$
\begin{array}{r}
\mathcal{D}_{D_{\epsilon}}^{i_{\omega}} \psi(s):=\int_{\partial D_{\epsilon}}\left(\frac{\partial}{\partial \nu(t)} \Gamma^{0}(s, t)\right) \psi(t) d t, \\
\mathcal{D}_{D_{\epsilon}}^{d_{\omega}} \psi(s):=\int_{\partial D_{\epsilon}}\left(\frac{\partial}{\partial \nu(t)}\left[\Gamma^{\omega}(s, t)-\Gamma^{0}(s, t)\right]\right) \psi(t) d t .
\end{array}
$$

With this definition, $\frac{1}{2} I+\mathcal{D}_{D_{\epsilon}}^{i_{\omega}}: L^{2}\left(\partial D_{\epsilon}\right) \rightarrow L^{2}\left(\partial D_{\epsilon}\right)$ is invertible, see [20,26, 27. Hence, $\frac{1}{2} I+\mathcal{D}_{D_{\epsilon}}=$ $\left(\frac{1}{2} I+\mathcal{D}_{D_{\epsilon}}^{i_{\omega}}\right)\left(I+\left(\frac{1}{2} I+\mathcal{D}_{D_{\epsilon}}^{i_{\omega}}\right)^{-1} \mathcal{D}_{D_{\epsilon}}^{d_{\omega}}\right)$ and so

$$
\begin{aligned}
\|\left(\frac{1}{2} I\right. & \left.+\mathcal{D}_{D_{\epsilon}}\right)^{-1} \|_{\mathcal{L}\left(L^{2}\left(\partial D_{\epsilon}\right), L^{2}\left(\partial D_{\epsilon}\right)\right)} \\
& =\left\|\left(I+\left(\frac{1}{2} I+\mathcal{D}_{D_{\epsilon}}^{i_{\omega}}\right)^{-1} \mathcal{D}_{D_{\epsilon}}^{d_{\omega}}\right)^{-1}\left(\frac{1}{2} I+\mathcal{D}_{D_{\epsilon}}^{i_{\omega}}\right)^{-1}\right\|_{\mathcal{L}\left(L^{2}\left(\partial D_{\epsilon}\right), L^{2}\left(\partial D_{\epsilon}\right)\right)}\left\|\left(\frac{1}{2} I+\mathcal{D}_{D_{\epsilon}}^{i_{\omega}}\right)^{-1}\right\|_{\mathcal{L}\left(L^{2}\left(\partial D_{\epsilon}\right), L^{2}\left(\partial D_{\epsilon}\right)\right)} \\
& \leq\left\|\left(I+\left(\frac{1}{2} I+\mathcal{D}_{D_{\epsilon}}^{i_{\omega}}\right)^{-1} \mathcal{D}_{D_{\epsilon}}^{d_{\omega}}\right)^{-1}\right\|_{\mathcal{L}\left(L^{2}\left(\partial D_{\epsilon}\right), L^{2}\left(\partial D_{\epsilon}\right)\right)} \|
\end{aligned}
$$

So, to estimate the operator norm of $\left(\frac{1}{2} I+\mathcal{D}_{D_{\epsilon}}\right)^{-1}$ one needs to estimate the operator norm of $\left(I+\left(\frac{1}{2} I+\mathcal{D}_{D_{\epsilon}}^{i_{\omega}}\right)^{-1} \mathcal{D}_{D_{\epsilon}}^{d_{\omega}}\right)^{-1}$. In particular one needs to have the knowledge about the operator norms of $\left(\frac{1}{2} I+\mathcal{D}_{D_{\epsilon}}^{i_{\omega}}\right)^{-1}$ and $\mathcal{D}_{D_{\epsilon}}^{d_{\omega}}$ to apply the Neumann series. For that purpose, we can estimate the operator norm of $\left(\frac{1}{2} I+\mathcal{D}_{D_{\epsilon}}^{i_{\omega}}\right)^{-1}$ from (2.29) by

$$
\left\|\left(\frac{1}{2} I+\mathcal{D}_{D_{\epsilon}}^{i_{\omega}}\right)^{-1}\right\|_{\mathcal{L}\left(L^{2}\left(\partial D_{\epsilon}\right), L^{2}\left(\partial D_{\epsilon}\right)\right)}=\left\|\left(\frac{1}{2} I+\mathcal{D}_{B}^{i_{\omega}}\right)^{-1}\right\|_{\mathcal{L}\left(L^{2}(\partial B), L^{2}(\partial B)\right)} .
$$


Here $\mathcal{D}_{B}^{i_{\omega}} \hat{\psi}(\xi):=\int_{\partial B}\left(\frac{\partial}{\partial \nu(\eta)} \Gamma^{0}(\xi, \eta)\right) \hat{\psi}(\eta) d \eta$. From the definition of the operator $\mathcal{D}_{D_{\epsilon}}^{d_{k}}$ in (2.33), we deduce that

$$
\begin{aligned}
\mathcal{D}_{D_{\epsilon}}^{d_{\omega}} \psi(s)= & \int_{\partial B}\left(\frac{\partial}{\partial \nu(\eta)}\left[\Gamma^{\epsilon \omega}(\xi, \eta)-\Gamma^{0}(\xi, \eta)\right]\right) \hat{\psi}(\eta) d \eta \\
= & \int_{\partial B}\left[\lambda\left(\operatorname{div}_{\eta}\left[\Gamma^{\epsilon \omega}(\xi, \eta)-\Gamma^{0}(\xi, \eta)\right]\right) N_{\eta}\right. \\
& \left.\quad+\mu\left(\nabla_{\eta}\left[\Gamma^{\epsilon \omega}(\xi, \eta)-\Gamma^{0}(\xi, \eta)\right]+\left(\nabla_{\eta}\left[\Gamma^{\epsilon \omega}(\xi, \eta)-\Gamma^{0}(\xi, \eta)\right]\right)^{\top}\right) N_{\eta}\right] \hat{\psi}(\eta) d \eta \\
= & \int_{\partial B}\left[\lambda I_{1} \otimes N_{\eta}+\mu\left(I_{2}+I_{2}^{\top}\right) N_{\eta}\right] \hat{\psi}(\eta) d \eta
\end{aligned}
$$

where the vector $I_{1}$ and the third order tensor $I_{2}$ are estimated by using (2.3) and (2.4) as

$$
\begin{aligned}
I_{1}= & -\frac{\epsilon^{2}}{4 \pi} \sum_{l=2}^{\infty} \frac{\epsilon^{l-2} i^{l}}{l !(l+2)} \frac{(l-1)}{\omega^{2}}\left[-2 \kappa_{s^{\omega}}^{l+2}+(l+4) \kappa_{p^{\omega}}^{l+2}\right]|\xi-\eta|^{l-3}(\xi-\eta), \\
I_{2}= & -\frac{\epsilon^{2}}{4 \pi} \sum_{l=2}^{\infty} \frac{\epsilon^{l-2} i^{l}}{l !(l+2)} \frac{(l-1)}{\omega^{2}}\left[\left((l+1) \kappa_{s^{\omega}}^{l+2}+\kappa_{p^{\omega}}^{l+2}\right)|\xi-\eta|^{l-3}(\xi-\eta) \otimes \mathbf{I}\right. \\
& \left.-\left(\kappa_{s^{\omega}}^{l+2}-\kappa_{p^{\omega}}^{l+2}\right)|\xi-\eta|^{l-3}\left((l-3)|\xi-\eta|^{-2} \otimes^{3}(\xi-\eta)+\mathbf{I} \otimes(\xi-\eta)+(\xi-\eta) \otimes \mathbf{I}\right)\right] .
\end{aligned}
$$

Using the observation ' $\left\||x|^{p}\right\|_{L^{2}(D)} \leq\|x\|_{L^{2}(D)}^{p}|D|^{\frac{1-p}{2}}$, we obtain

$$
\begin{aligned}
& \left|\mathcal{D}_{D_{\epsilon}}^{d_{\omega}} \psi(s)\right| \\
& \leq \lambda \frac{\epsilon^{2}}{4 \pi} \sum_{l=2}^{\infty} \frac{\epsilon^{l-2}}{l !(l+2)} \frac{(l-1)}{\omega^{2}}\left(2 \kappa_{s^{\omega}}^{l+2}+(l+4) \kappa_{p^{\omega}}^{l+2}\right) \int_{\partial B}|\xi-\eta|^{l-2}|\hat{\psi}(\eta)| d \eta+ \\
& 2 \mu \frac{\epsilon^{2}}{4 \pi}\left[\sum_{l=3}^{\infty} \frac{\epsilon^{l-2}}{l !(l+2)} \frac{(l-1)}{\omega^{2}}\left(2 l \kappa_{s^{\omega}}^{l+2}+l \kappa_{p^{\omega}}^{l+2}\right) \int_{\partial B}|\xi-\eta|^{l-2}|\hat{\psi}(\eta)| d \eta+\frac{\left(6 \kappa_{s^{\omega}}^{4}+4 \kappa_{p^{\omega}}^{4}\right)}{8 \omega^{2}} \int_{\partial B}|\hat{\psi}(\eta)| d \eta\right] \\
& \leq \lambda \frac{\epsilon^{2}}{4 \pi}\|\hat{\psi}\|_{L^{2}(\partial B)}|\partial B|^{\frac{1}{2}}\left[\sum_{l=2}^{\infty} \frac{\epsilon^{l-2}}{l !(l+2)} \frac{(l-1)}{\omega^{2}}\left(2 \kappa_{s^{\omega}}^{l+2}+(l+4) \kappa_{p^{\omega}}^{l+2}\right)\|\xi-\cdot\|_{L^{2}(\partial B)}^{l-2}|\partial B|^{\frac{2-l}{2}}\right]+ \\
& 2 \mu \frac{\epsilon^{2}}{4 \pi}\|\hat{\psi}\|_{L^{2}(\partial B)}|\partial B|^{\frac{1}{2}}\left[\sum_{l=2}^{\infty} \frac{\epsilon^{l-2}}{(l-2) !(l+2)} \frac{1}{\omega^{2}}\left(2 \kappa_{s^{\omega}}^{l+2}+\kappa_{p^{\omega}}^{l+2}\right)\|\xi-\cdot\|_{L^{2}(\partial B)}^{l-2}|\partial B|^{\frac{2-l}{2}}+\frac{\left(\kappa_{s^{\omega}}^{4}+\kappa_{p^{\omega}}^{4}\right)}{4 \omega^{2}}\right] \\
& \leq \omega^{2} \frac{\epsilon^{2}}{4 \pi}\|\hat{\psi}\|_{L^{2}(\partial B)}|\partial B|^{\frac{1}{2}}\left(\frac{1}{c_{s}^{4}}\left[\frac{\mu}{2}+(\lambda+4 \mu) \sum_{l=0}^{\infty}\left(\frac{1}{2} \epsilon \kappa_{s^{\omega}}\|\xi-\cdot\|_{L^{2}(\partial B)}|\partial B|^{\frac{-1}{2}}\right)^{l}\right]\right. \\
& \left.+\frac{1}{c_{p}^{4}}\left[\frac{\mu}{2}+(3 \lambda+2 \mu) \sum_{l=0}^{\infty}\left(\frac{1}{2} \epsilon \kappa_{p^{\omega}}\|\xi-\cdot\|_{L^{2}(\partial B)}|\partial B|^{\frac{-1}{2}}\right)^{l}\right]\right) \\
& =\omega^{2} \frac{\epsilon^{2}}{4 \pi}\|\hat{\psi}\|_{L^{2}(\partial B)}|\partial B|^{\frac{1}{2}}\left(\frac{1}{c_{s}^{4}}\left[\frac{\mu}{2}+\frac{\lambda+4 \mu}{1-\frac{1}{2} \epsilon \kappa_{s^{\omega}}\|\xi-\cdot\|_{L^{2}(\partial B)}|\partial B|^{\frac{-1}{2}}}\right]\right. \\
& \left.+\frac{1}{c_{p}^{4}}\left[\frac{\mu}{2}+\frac{3 \lambda+2 \mu}{1-\frac{1}{2} \epsilon \kappa_{p^{\omega}}\|\xi-\cdot\|_{L^{2}(\partial B)}|\partial B|^{\frac{-1}{2}}}\right]\right), \text { for } \epsilon<\frac{2 \min \left\{c_{s}, c_{p}\right\}}{\omega_{\max } \max _{m} \operatorname{diam}\left(B_{m}\right)} \\
& \leq \grave{C}_{1} \omega^{2} \epsilon^{2}\|\hat{\psi}\|_{L^{2}(\partial B)}, \text { for } \epsilon \leq \frac{\min \left\{c_{s}, c_{p}\right\}}{\omega_{\max } \max _{m} \operatorname{diam}\left(B_{m}\right)} \text {, }
\end{aligned}
$$


with $\grave{C}_{1}:=\frac{|\partial B|^{\frac{1}{2}}}{4 \pi}\left[\frac{4 \lambda+17 \mu}{2 c_{s}^{4}}+\frac{12 \lambda+9 \mu}{2 c_{p}^{4}}\right]$. From this we obtain,

$$
\begin{aligned}
\left\|\mathcal{D}_{D_{\epsilon}}^{d_{\omega}} \psi\right\|_{L^{2}\left(\partial D_{\epsilon}\right)}^{2} & =\int_{\partial D_{\epsilon}}\left|\mathcal{D}_{D_{\epsilon}}^{d_{\omega}} \psi(s)\right|^{2} d s \\
& \stackrel{\leq}{2.39}) \\
& \int_{\partial D_{\epsilon}}\left[\grave{C}_{1} \omega^{2} \epsilon^{2}\|\hat{\psi}\|_{L^{2}(\partial B)}\right]^{2} d s \\
& =\grave{C}_{1}^{2} \omega^{4} \epsilon^{6}|\partial B|\|\hat{\psi}\|_{L^{2}(\partial B)}^{2} .
\end{aligned}
$$

Hence

$$
\left\|\mathcal{D}_{D_{\epsilon}}^{d_{\omega}} \psi\right\|_{L^{2}\left(\partial D_{\epsilon}\right)} \leq \grave{C}_{1} \omega^{2} \epsilon^{3}|\partial B|^{\frac{1}{2}}\|\hat{\psi}\|_{L^{2}(\partial B)}
$$

We estimate the norm of the operator $\mathcal{D}_{D_{\epsilon}}^{d_{\omega}}$ as

$$
\begin{aligned}
& \left\|\mathcal{D}_{D_{\epsilon}}^{d_{\omega}}\right\|_{\mathcal{L}\left(L^{2}\left(\partial D_{\epsilon}\right), L^{2}\left(\partial D_{\epsilon}\right)\right)} \quad=\quad \operatorname{Sup}_{\substack{\operatorname{Sup}^{2}(\neq 0) \in L^{2}\left(\partial D_{\epsilon}\right) \\
\left\|\mathcal{D}_{D_{\epsilon}}^{d_{\omega}} \psi\right\|_{L^{2}\left(\partial D_{\epsilon}\right)}}}
\end{aligned}
$$

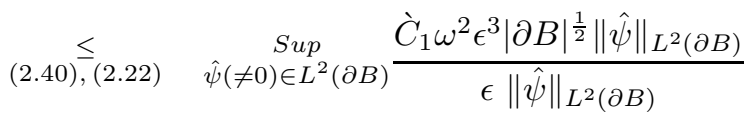

$$
\begin{aligned}
& =\quad \grave{C}_{1} \omega^{2} \epsilon^{2}|\partial B|^{\frac{1}{2}} .
\end{aligned}
$$

Hence, we get

$$
\begin{array}{cl}
\left\|\left(\frac{1}{2} I+\mathcal{D}_{D_{\epsilon}}^{i_{\omega}}\right)^{-1} \mathcal{D}_{D_{\epsilon}}^{d_{\omega}}\right\|_{\mathcal{L}\left(L^{2}\left(\partial D_{\epsilon}\right), L^{2}\left(\partial D_{\epsilon}\right)\right)} & \left\|\left(\frac{1}{2} I+\mathcal{D}_{D_{\epsilon}}^{i_{\omega}}\right)^{-1}\right\|_{\mathcal{L}\left(L^{2}\left(\partial D_{\epsilon}\right), L^{2}\left(\partial D_{\epsilon}\right)\right)}\left\|\mathcal{D}_{D_{\epsilon}}^{d_{\omega}}\right\|_{\mathcal{L}\left(L^{2}\left(\partial D_{\epsilon}\right), L^{2}\left(\partial D_{\epsilon}\right)\right)} \\
\leq & \left\|\left(\frac{1}{2} I+\mathcal{D}_{B}^{i_{\omega}}\right)^{-1}\right\|_{\grave{C}_{1} \omega^{2} \epsilon^{2}|\partial B|^{\frac{1}{2}}} \\
\leq & \grave{C}_{2} \omega^{2} \epsilon^{2}
\end{array}
$$

where $\grave{C}_{2}:=\grave{C}_{1}|\partial B|^{\frac{1}{2}}\left\|\left(\frac{1}{2} I+\mathcal{D}_{B}^{i_{\omega}}\right)^{-1}\right\|_{\mathcal{L}\left(L^{2}(\partial B), L^{2}(\partial B)\right)}$. Assuming $\epsilon$ to satisfy the condition $\epsilon<\frac{1}{\sqrt{\grave{C}_{2}} \omega_{\max }}$, then $\left\|\left(\frac{1}{2} I+\mathcal{D}_{D_{\epsilon}}^{i_{\omega}}\right)^{-1} \mathcal{D}_{D_{\epsilon}}^{d_{\omega}}\right\|_{\mathcal{L}\left(L^{2}\left(\partial D_{\epsilon}\right), L^{2}\left(\partial D_{\epsilon}\right)\right)}<1$ and hence by using the Neumann series we obtain the following bound

$$
\begin{aligned}
& \left\|\left(I+\left(\frac{1}{2} I+\mathcal{D}_{D_{\epsilon}}^{i_{\omega}}\right)^{-1} \mathcal{D}_{D_{\epsilon}}^{d_{\omega}}\right)^{-1}\right\|_{\mathcal{L}\left(L^{2}\left(\partial D_{\epsilon}\right), L^{2}\left(\partial D_{\epsilon}\right)\right)} \leq \frac{1}{1-\|\left(\frac{1}{2} I+\mathcal{D}_{D_{\epsilon} i^{i_{\omega}}}^{-1} \mathcal{D}_{D_{\epsilon} d_{\omega}}^{d_{L}} \|_{\mathcal{L}\left(L^{2}\left(\partial D_{\epsilon}\right), L^{2}\left(\partial D_{\epsilon}\right)\right)}\right.} \\
& \stackrel{\leq}{(2.42)} \quad \grave{C}_{3}:=\frac{1}{1-\grave{C}_{2} \omega^{2} \epsilon^{2}} \text {. }
\end{aligned}
$$

By substituting the above and (2.35) in (2.34), we obtain the required result (2.31).

\subsubsection{The multiple obstacle case}


Lemma 2.6. For each $k>0$ and for every $n \in \mathbb{Z}^{+}$with $n \geq k e^{2}[=: N(k)]$ we have $n ! \geq k^{n-1}$.

Proof. of Lemma 2.6. The result is true for $n=1$. The proof goes as follows for $n>1$ :

$$
\begin{aligned}
n \geq k e^{2} & \Longrightarrow \quad \ln k \leq \ln n-2 \\
4 & \Longrightarrow \quad \ln k \leq \frac{\ln \sqrt{2 \pi}-n}{n-1}+\frac{\left(n+\frac{1}{2}\right)}{n-1} \ln n \\
& \Longrightarrow \quad(n-1) \ln k \leq \ln \sqrt{2 \pi}+\left(n+\frac{1}{2}\right) \ln n-n \\
& \Longrightarrow \quad k^{n-1} \leq \sqrt{2 \pi n}\left(\frac{n}{e}\right)^{n}
\end{aligned}
$$

Now, we obtain the result using Stirlings approximation $n ! \sim \sqrt{2 \pi n}\left(\frac{n}{e}\right)^{n}$, precisely $\sqrt{2 \pi n}\left(\frac{n}{e}\right)^{n} \leq n !$, see 32 for instance.

Proposition 2.7. For $m, j=1,2, \ldots, M$, the operator $\mathcal{D}_{m j}: L^{2}\left(\partial D_{j}\right) \rightarrow L^{2}\left(\partial D_{m}\right)$ defined in Proposition 2.1, see (2.11), enjoys the following estimates,

- For $j=m$,

$$
\begin{gathered}
\left\|\left(\frac{1}{2} I+\mathcal{D}_{m m}\right)^{-1}\right\|_{\mathcal{L}\left(L^{2}\left(\partial D_{m}\right), L^{2}\left(\partial D_{m}\right)\right)} \leq \grave{C}_{6 m}, \\
\text { where } \grave{C}_{6 m}:=\frac{4 \pi\left\|\left(\frac{1}{2} I+\mathcal{D}_{B_{m}}^{i_{\omega}}\right)^{-1}\right\|_{\mathcal{L}\left(L^{2}\left(\partial B_{m}\right), L^{2}\left(\partial B_{m}\right)\right)}}{4 \pi-\left[\frac{4 \lambda+17 \mu}{2 c_{s}^{4}}+\frac{12 \lambda+9 \mu}{2 c_{p}^{4}}\right] \omega^{2} \epsilon^{2}\left|\partial B_{m}\right|\left\|\left(\frac{1}{2} I+\mathcal{D}_{B_{m}}^{i_{\omega}}\right)^{-1}\right\|_{\mathcal{L}\left(L^{2}\left(\partial B_{m}\right), L^{2}\left(\partial B_{m}\right)\right)}} .
\end{gathered}
$$

- For $j \neq m$,

$$
\left\|\mathcal{D}_{m j}\right\|_{\mathcal{L}\left(L^{2}\left(\partial D_{j}\right), L^{2}\left(\partial D_{m}\right)\right)} \leq\left[\frac{\tilde{C}_{7}}{d^{2}}+\tilde{C}_{8}\right] \frac{1}{4 \pi}|\partial B \beta| \epsilon^{2}
$$

where

$$
\begin{gathered}
|\partial B|:=\max _{m} \partial B_{m}, \quad \tilde{C}_{7}:=\left(\frac{\lambda+6 \mu}{c_{s}^{2}}+\frac{2 \lambda+6 \mu}{c_{p}^{2}}\right) \text { and } \\
\tilde{C}_{8}:=\frac{\omega^{2}}{c_{s}^{4}}\left(\frac{\mu}{2}+(\lambda+4 \mu) \frac{1-\left(\frac{1}{2} \kappa_{s} \omega \operatorname{diam}(\Omega)\right)^{N_{\Omega}}}{1-\frac{1}{2} \kappa_{s} \omega \operatorname{diam}(\Omega)}+(\lambda+4 \mu) \frac{1}{2^{N_{\Omega}-1}}\right) \\
+\frac{\omega^{2}}{c_{p}^{4}}\left(\frac{\mu}{2}+\frac{(3 \lambda+4 \mu)}{2} \frac{1-\left(\frac{1}{2} \kappa_{p^{\omega}} \operatorname{diam}(\Omega)\right)^{N_{\Omega}}}{1-\frac{1}{2} \kappa_{p^{\omega}} \operatorname{diam}(\Omega)}+\frac{(3 \lambda+4 \mu)}{2} \frac{1}{2^{N_{\Omega}-1}}\right)
\end{gathered}
$$

with $N_{\Omega}=\left[2 \operatorname{diam}(\Omega) \max \left\{\kappa_{s^{\omega}}, \kappa_{p^{\omega}}\right\} e^{2}\right]$, where [.] denotes the integral part.

Proof. of Proposition 2.7. The estimate (2.43) is nothing but (2.31) of Lemma 2.5, replacing $B$ by $B_{m}, z$ by $z_{m}$ and $D_{\epsilon}$ by $D_{m}$ respectively. It remains to prove the estimate (2.44). We have

$$
\left\|\mathcal{D}_{m j}\right\|_{\mathcal{L}\left(L^{2}\left(\partial D_{j}\right), L^{2}\left(\partial D_{m}\right)\right)}=\underset{\psi(\neq 0) \in L^{2}\left(\partial D_{j}\right)}{\operatorname{Sup}_{m j}} \frac{\left\|\mathcal{D}_{m j} \psi\right\|_{L^{2}\left(\partial D_{m}\right)}}{\|\psi\|_{L^{2}\left(\partial D_{j}\right)}} .
$$

\footnotetext{
${ }^{4}$ Since, $\frac{\left(n+\frac{1}{2}\right)}{n-1}>1, \frac{\ln \sqrt{2 \pi}}{n-1}>0$ and $0<\frac{n}{n-1}<2$ for $n>1$.
} 
Let $\psi \in L^{2}\left(\partial D_{j}\right)$ then for $s \in \partial D_{m}$, we have

$$
\begin{aligned}
\mathcal{D}_{m j} \psi(s) & =\int_{\partial D_{j}} \frac{\partial \Gamma^{\omega}(s, t)}{\partial \nu_{j}(t)} \psi(t) d t \\
& =\int_{\partial D_{j}}\left[\lambda\left(\operatorname{div}_{t}\left[\Gamma^{\omega}(s, t)\right]\right) N_{t}+\mu\left(\nabla_{t}\left[\Gamma^{\omega}(s, t)\right]+\left(\nabla_{t}\left[\Gamma^{\omega}(s, t)\right]\right)^{\top}\right) N_{t}\right] \psi(t) d t \\
& =\int_{\partial D_{j}}\left[\lambda I_{1^{\prime}} \otimes N_{t}+\mu\left(I_{2^{\prime}}+I_{2^{\prime}}^{\top}\right) N_{t}\right] \psi(t) d t,
\end{aligned}
$$

where the vector $I_{1^{\prime}}$ and the third order tensor $I_{2^{\prime}}$ are given by

$$
\begin{aligned}
I_{1^{\prime}}= & -\frac{1}{4 \pi} \sum_{l=0}^{\infty} \frac{i^{l}}{l !(l+2)} \frac{(l-1)}{\omega^{2}}\left[-2 \kappa_{s^{\omega}}^{l+2}+(l+4) \kappa_{p^{\omega}}^{l+2}\right]|s-t|^{l-3}(s-t), \\
I_{2^{\prime}}= & -\frac{1}{4 \pi} \sum_{l=0}^{\infty} \frac{i^{l}}{l !(l+2)} \frac{(l-1)}{\omega^{2}}\left[\left((l+1) \kappa_{s^{\omega}}^{l+2}+\kappa_{p^{\omega}}^{l+2}\right)|s-t|^{l-3}(s-t) \otimes \mathbf{I}\right. \\
& \left.-\left(\kappa_{s^{\omega}}^{l+2}-\kappa_{p^{\omega}}^{l+2}\right)|s-t|^{l-3}\left((l-3)|s-t|^{-2} \otimes^{3}(s-t)+\mathbf{I} \otimes(\mathrm{s}-\mathrm{t})+(\mathrm{s}-\mathrm{t}) \otimes \mathbf{I}\right)\right] .
\end{aligned}
$$

Then, by using Lemma 2.6, we estimate

$$
\begin{aligned}
& \left|\mathcal{D}_{m j} \psi(s)\right| \leq \frac{\lambda}{4 \pi}\left[\frac{\left(\kappa_{s^{\omega}}^{2}+2 \kappa_{p^{\omega}}^{2}\right)}{\omega^{2}} \int_{\partial D_{j}}|s-t|^{-2}|\psi(t)| d t\right. \\
& \left.+\sum_{l=2}^{\infty} \frac{1}{l !(l+2)} \frac{(l-1)}{\omega^{2}}\left(2 \kappa_{s^{\omega}}^{l+2}+(l+4) \kappa_{p^{\omega}}^{l+2}\right) \int_{\partial D_{j}}|s-t|^{l-2}|\psi(t)| d t\right] \\
& +\frac{2 \mu}{4 \pi}\left[\frac{3\left(\kappa_{s^{\omega}}^{2}+\kappa_{p^{\omega}}^{2}\right)}{\omega^{2}} \int_{\partial D_{j}}|s-t|^{-2}|\psi(t)| d t+\frac{\left(6 \kappa_{s^{\omega}}^{4}+4 \kappa_{p^{\omega}}^{4}\right)}{8 \omega^{2}} \int_{\partial D_{j}}|\psi(t)| d t\right. \\
& \left.+\sum_{l=3}^{\infty} \frac{1}{l !(l+2)} \frac{(l-1)}{\omega^{2}}\left(2 l \kappa_{s^{\omega}}^{l+2}+l \kappa_{p^{\omega}}^{l+2}\right) \int_{\partial D_{j}}|s-t|^{l-2}|\psi(t)| d t\right] \\
& \leq \frac{\lambda}{4 \pi}\|\psi\|_{L^{2}\left(D_{j}\right)}\left|\partial D_{j}\right|^{\frac{1}{2}} \\
& {\left[\frac{\left(\kappa_{s^{\omega}}^{2}+2 \kappa_{p^{\omega}}^{2}\right)}{\omega^{2}} \frac{1}{d_{m j}^{2}}+\sum_{l=2}^{\infty} \frac{1}{l !(l+2)} \frac{(l-1)}{\omega^{2}}\left(2 \kappa_{s^{\omega}}^{l+2}+(l+4) \kappa_{p^{\omega}}^{l+2}\right) \operatorname{diam}(\Omega)^{l-2}\right]} \\
& +\frac{2 \mu}{4 \pi}\|\psi\|_{L^{2}\left(D_{j}\right)}\left|\partial D_{j}\right|^{\frac{1}{2}} \\
& {\left[\frac{3\left(\kappa_{s^{\omega}}^{2}+\kappa_{p^{\omega}}^{2}\right)}{\omega^{2}} \frac{1}{d_{m j}^{2}}+\frac{\left(\kappa_{s^{\omega}}^{4}+\kappa_{p^{\omega}}^{4}\right)}{4 \omega^{2}}+\sum_{l=2}^{\infty} \frac{1}{(l-2) !(l+2)} \frac{1}{\omega^{2}}\left(2 \kappa_{s^{\omega}}^{l+2}+\kappa_{p^{\omega}}^{l+2}\right) \operatorname{diam}(\Omega)^{l-2}\right]} \\
& \leq \frac{\lambda}{4 \pi}\|\psi\|_{L^{2}\left(D_{j}\right)}\left|\partial D_{j}\right|^{\frac{1}{2}}\left[\frac{1}{d_{m j}^{2}}\left(\frac{1}{c_{s}^{2}}+\frac{2}{c_{p}^{2}}\right)+\frac{\omega^{2}}{c_{s}^{4}} \sum_{l=2}^{N_{\Omega}+1}\left(\frac{1}{2} \kappa_{s^{\omega}} \operatorname{diam}(\Omega)\right)^{l-2}+\frac{\omega^{2}}{c_{s}^{4}}\right. \\
& \left.\sum_{l=N_{\Omega}+2}^{\infty}\left(\frac{1}{2}\right)^{l-2}+\frac{3}{2} \frac{\omega^{2}}{c_{p}^{4}} \sum_{l=2}^{N_{\Omega}+1}\left(\frac{1}{2} \kappa_{p^{\omega}} \operatorname{diam}(\Omega)\right)^{l-2}+\frac{3}{2} \frac{\omega^{2}}{c_{p}^{4}} \sum_{l=N_{\Omega}+2}^{\infty}\left(\frac{1}{2} \kappa_{p^{\omega}} \operatorname{diam}(\Omega)\right)^{l-2}\right] \\
& +\frac{2 \mu}{4 \pi}\|\psi\|_{L^{2}\left(D_{j}\right)}\left|\partial D_{j}\right|^{\frac{1}{2}}\left[\frac{3}{d_{m j}^{2}}\left(\frac{1}{c_{s}^{2}}+\frac{1}{c_{p}^{2}}\right)+\frac{\omega^{2}}{4}\left(\frac{1}{c_{s}^{4}}+\frac{1}{c_{p}^{4}}\right)+\frac{2 \omega^{2}}{c_{s}^{4}} \sum_{l=2}^{N_{\Omega}+1}\left(\frac{1}{2} \kappa_{s} \omega \operatorname{diam}(\Omega)\right)^{l-2}\right.
\end{aligned}
$$




$$
\begin{aligned}
& +\frac{2 \omega^{2}}{c_{s}^{4}} \sum_{l=N_{\Omega}+2}^{\infty}\left(\frac{1}{2}\right)^{l-2}+\frac{\omega^{2}}{c_{p}^{4}} \sum_{l=2}^{N_{\Omega}+1}\left(\frac{1}{2} \kappa_{p^{\omega}} \operatorname{diam}(\Omega)\right)^{l-2}+\frac{\omega^{2}}{c_{p}^{4}} \sum_{l=N_{\Omega}+2}^{\infty}\left(\frac{1}{2}\right)^{l-2 \rrbracket 6} \\
& =\frac{1}{4 \pi}\|\psi\|_{L^{2}\left(D_{j}\right)}\left|\partial D_{j}\right|^{\frac{1}{2}}\left[\frac{1}{d_{m j}^{2}}\left(\frac{\lambda+6 \mu}{c_{s}^{2}}+\frac{2 \lambda+6 \mu}{c_{p}^{2}}\right)\right. \\
& +\frac{\omega^{2}}{c_{s}^{4}}\left(\frac{\mu}{2}+(\lambda+4 \mu) \sum_{l=0}^{N_{\Omega}-1}\left(\frac{1}{2} \kappa_{s} \omega \operatorname{diam}(\Omega)\right)^{l}+(\lambda+4 \mu) \sum_{l=N_{\Omega}}^{\infty}\left(\frac{1}{2}\right)^{l}\right) \\
& \left.+\frac{\omega^{2}}{c_{p}^{4}}\left(\frac{\mu}{2}+\frac{(3 \lambda+4 \mu)}{2} \sum_{l=0}^{N_{\Omega}-1}\left(\frac{1}{2} \kappa_{p^{\omega}} \operatorname{diam}(\Omega)\right)^{l}+\frac{(3 \lambda+4 \mu)}{2} \sum_{l=N_{\Omega}}^{\infty}\left(\frac{1}{2}\right)^{l}\right)\right] \\
& \leq \frac{\epsilon\|\psi\|_{L^{2}\left(D_{j}\right)}\left|\partial B_{j}\right|^{\frac{1}{2}}}{4 \pi}\left[\frac{1}{d_{m j}^{2}}\left(\frac{\lambda+6 \mu}{c_{s}^{2}}+\frac{2 \lambda+6 \mu}{c_{p}^{2}}\right)\right. \\
& +\frac{\omega^{2}}{c_{s}^{4}}\left(\frac{\mu}{2}+(\lambda+4 \mu) \frac{1-\left(\frac{1}{2} \kappa_{s^{\omega}} \operatorname{diam}(\Omega)\right)^{N_{\Omega}}}{1-\frac{1}{2} \kappa_{s^{\omega}} \operatorname{diam}(\Omega)}+(\lambda+4 \mu) \frac{1}{2^{N_{\Omega}-1}}\right) \\
& \left.+\frac{\omega^{2}}{c_{p}^{4}}\left(\frac{\mu}{2}+\frac{(3 \lambda+4 \mu)}{2} \frac{1-\left(\frac{1}{2} \kappa_{p^{\omega}} \operatorname{diam}(\Omega)\right)^{N_{\Omega}}}{1-\frac{1}{2} \kappa_{p^{\omega}} \operatorname{diam}(\Omega)}+\frac{(3 \lambda+4 \mu)}{2} \frac{1}{2^{N_{\Omega}-1}}\right)\right] \\
& =\frac{1}{4 \pi}\left[\frac{\tilde{C}_{7}}{d_{m j}^{2}}+\tilde{C}_{8}\right] \epsilon\left|\partial B_{j}\right|^{\frac{1}{2}}\|\psi\|_{L^{2}\left(\partial D_{j}\right)}
\end{aligned}
$$

form which, we get

$$
\begin{aligned}
\left\|\mathcal{D}_{m j} \psi\right\|_{L^{2}\left(\partial D_{m}\right)} & =\left(\int_{\partial D_{m}}\left|\mathcal{D}_{m j} \psi(s)\right|^{2} d s\right)^{\frac{1}{2}} \\
& \stackrel{\leq}{2.49)}\left[\frac{\tilde{C}_{7}}{d_{m j}^{2}}+\tilde{C}_{8}\right] \frac{1}{4 \pi} \epsilon\left|\partial B_{j}\right|^{\frac{1}{2}}\|\psi\|_{L^{2}\left(\partial D_{j}\right)}\left(\int_{\partial D_{m}} d s\right)^{\frac{1}{2}} \\
& =\left[\frac{\tilde{C}_{7}}{d_{m j}^{2}}+\tilde{C}_{8}\right] \frac{1}{4 \pi} \epsilon^{2}\left|\partial B_{j}\right|^{\frac{1}{2}}\left|\partial B_{m}\right|^{\frac{1}{2}}\|\psi\|_{L^{2}\left(\partial D_{j}\right)} .
\end{aligned}
$$

Substitution of (2.50) in (2.45) gives us

$$
\begin{aligned}
\left\|\mathcal{D}_{m j}\right\|_{\mathcal{L}\left(L^{2}\left(\partial D_{j}\right), L^{2}\left(\partial D_{m}\right)\right)} & \leq\left[\frac{\tilde{C}_{7}}{d_{m j}^{2}}+\tilde{C}_{8}\right] \frac{1}{4 \pi d_{m j}^{2}} \epsilon^{2}\left|\partial B_{j}\right|^{\frac{1}{2}}\left|\partial B_{m}\right|^{\frac{1}{2}} \\
& \leq\left[\frac{\tilde{C}_{7}}{d^{2}}+\tilde{C}_{8}\right] \frac{1}{4 \pi}|\partial \mathrm{B}| \epsilon^{2} .
\end{aligned}
$$

End of the proof of Proposition 2.2. By substituting (2.43) in (2.17) and (2.44) in (2.16), we obtain

$$
\left\|\left(\frac{1}{2} I+D L\right)^{-1}\right\| \leq \max _{m=1}^{M} \grave{C}_{6 m}
$$

and

$$
\|D K\| \leq \frac{M-1}{4 \pi}\left[\frac{\tilde{C}_{7}}{d^{2}}+\tilde{C}_{8}\right]|\partial \mathrm{B}| \epsilon^{2} .
$$


Hence, 2.52) and (2.51) jointly provide

$$
\left\|\left(\frac{1}{2} I+D L\right)^{-1}\right\|\|D K\| \leq \underbrace{\frac{M-1}{4 \pi}\left[\frac{\tilde{C}_{7}}{d^{2}}+\tilde{C}_{8}\right]\left(\max _{m=1}^{M} \grave{C}_{6 m}\right)|\partial \mathrm{B}| \epsilon^{2}}_{=: \grave{C}_{s}},
$$

By imposing the condition $\left\|\left(\frac{1}{2} I+D L\right)^{-1}\right\|\|D K\|<1$, we get the following from (2.15) and (2.18] 2.19)

$$
\begin{aligned}
& \left\|\sigma_{m}\right\|_{L^{2}\left(\partial D_{m}\right)} \leq\|\sigma\| \leq \frac{\left\|\left(\frac{1}{2} I+D L\right)^{-1}\right\|}{1-\left\|\left(\frac{1}{2} I+D L\right)^{-1}\right\|\|D K\|}\left\|U^{I n}\right\| \\
& \leq \quad \grave{C}_{p}\left\|\left(\frac{1}{2} I+D L\right)^{-1}\right\| \max _{m=1}^{M}\left\|U^{i}\right\|_{L^{2}\left(\partial D_{m}\right)} \quad\left(\grave{C}_{p} \geq \frac{1}{1-\grave{C}_{s}}\right) \\
& \underset{\text { (2.51) }}{<} \grave{\mathrm{C}}_{m=1}^{M}\left\|U^{i}\right\|_{L^{2}\left(\partial D_{m}\right)} \quad\left(\grave{\mathrm{C}}:=\grave{C}_{p} \max _{m=1}^{M} \grave{C}_{6 m}\right) \text {, }
\end{aligned}
$$

for all $m \in\{1,2, \ldots, M\}$. But, for the plane incident wave of the Lamé system, $U^{i}(x, \theta):=\alpha \theta e^{\left(i \omega x \cdot \theta / c_{p}\right)}+$ $\beta \theta^{\perp} e^{\left(i \kappa x \cdot \theta / c_{s}\right)}$, we have

$$
\left\|U^{i}\right\|_{L^{2}\left(\partial D_{m}\right)} \leq(|\alpha|+|\beta|) \epsilon\left|\partial B_{m}\right|^{\frac{1}{2}} \leq(|\alpha|+|\beta|) \epsilon|\partial \mathrm{B}|^{\frac{1}{2}}, \forall m=1,2, \ldots, M
$$

Now by substituting (2.55) in (2.54), for each $m=1, \ldots, M$, we obtain

$$
\left\|\sigma_{m}\right\|_{L^{2}\left(\partial D_{m}\right)} \leq \grave{\mathcal{C}}(\omega) \epsilon
$$

where $\grave{\mathcal{C}}(\omega):=\grave{\mathrm{C}}|\partial \mathrm{B}|^{\frac{1}{2}}(|\alpha|+|\beta|)$.

The condition $\left\|\left(\frac{1}{2} I+D L\right)^{-1}\right\|\|D K\|<1$ is satisfied if $\grave{C}_{s}<1$, i.e.

$$
\frac{M-1}{4 \pi}\left[\frac{\tilde{C}_{7}}{d^{2}}+\tilde{C}_{8}\right]|\partial \mathrm{B}|\left(\max _{m=1}^{M} \grave{C}_{6 m}\right) \epsilon^{2}<1 .
$$

The condition (2.57) reads as $\sqrt{M-1} \epsilon<\grave{c} d$ where we set

$$
\grave{c}:=\left[\frac{1}{4 \pi}\left[\tilde{C}_{7}+\tilde{C}_{8} d_{\max }^{2}\right]|\partial \mathrm{B}| \max _{m=1}^{M} \grave{C}_{6 m}\right]^{-\frac{1}{2}}
$$

and it serves our purpose in Proposition 2.2 and hence in Theorem 1.2

\subsection{The single layer potential representation and the total charge}

\subsubsection{The single layer potential representation}

For $m=1,2, \ldots, M$, let $U^{\sigma_{m}}$ be the solution of the problem

$$
\begin{cases}\left(\Delta^{e}+\omega^{2}\right) U^{\sigma_{m}}=0 & \text { in } D_{m} \\ U^{\sigma_{m}}=\sigma_{m} & \text { on } \partial D_{m} .\end{cases}
$$


The function $\sigma_{m}$ is in $H^{1}\left(\partial D_{m}\right)$, see Proposition 2.1. Hence $U^{\sigma_{m}} \in H^{\frac{3}{2}}\left(D_{m}\right)$ and then $\left.\frac{\partial U^{\sigma_{m}}}{\partial \nu_{m}}\right|_{\partial D_{m}} 18$ $L^{2}\left(\partial D_{m}\right)$. From Proposition 2.1, the solution of the problem (1.1]1.3) has the form

$$
U^{t}(x)=U^{i}(x)+\sum_{m=1}^{M} \int_{\partial D_{m}} \frac{\partial \Gamma^{\omega}(x, s)}{\partial \nu_{m}(s)} \sigma_{m}(s) d s, x \in \mathbb{R}^{3} \backslash\left(\bigcup_{m=1}^{M} \bar{D}_{m}\right) .
$$

It can be written in terms of single layer potanetial using Gauss's theorem as

$$
U^{t}(x)=U^{i}(x)+\sum_{m=1}^{M} \int_{\partial D_{m}} \Gamma^{\omega}(x, s) \frac{\partial U^{\sigma_{m}}(s)}{\partial \nu_{m}(s)} d s, x \in \mathbb{R}^{3} \backslash\left(\bigcup_{m=1}^{M} \bar{D}_{m}\right) .
$$

Indeed, by Betti's third identity,

$$
\begin{aligned}
\int_{\partial D_{m}} \frac{\partial \Gamma^{\omega}(x, s)}{\partial \nu_{m}(s)} \sigma_{m}(s) d s= & \int_{\partial D_{m}} \Gamma^{\omega}(x, s) \frac{\partial U^{\sigma_{m}}(s)}{\partial \nu_{m}(s)} d s \\
& +\int_{D_{m}}\left[U^{\sigma_{m}}(y) \Delta^{e} \Gamma^{\omega}(x, y)-\Gamma^{\omega}(x, y) \Delta^{e} U^{\sigma_{m}}(y)\right] d y .
\end{aligned}
$$

Lemma 2.8. For $m=1,2, \ldots, M, U^{\sigma_{m}}$, the solutions of the problem (2.58), satisfies the estimate

$$
\left\|\frac{\partial U^{\sigma_{m}}(s)}{\partial \nu_{m}(s)}\right\|_{H^{-1}\left(\partial D_{m}\right)} \leq C_{7}
$$

for some constant $C_{7}$ depending on $B_{m}$ through its Lipschitz character but it is independent of $\epsilon$.

Proof. of Lemma 2.8. For $m=1,2, \ldots, M$, we write

$$
\mathcal{U}^{m}(x):=U^{\sigma_{m}}\left(\epsilon x+z_{m}\right), \forall x \in B_{m} .
$$

Then we obtain

$$
\left\{\begin{array}{cl}
\left(\Delta^{e}+\epsilon^{2} \omega^{2}\right) \mathcal{U}^{m}(x) & =\epsilon^{2}\left(\Delta^{e}+\omega^{2}\right) U^{\sigma_{m}}\left(\epsilon x+z_{m}\right)=0, \text { for } x \in B_{m} \\
\mathcal{U}^{m}(\xi) & =U^{\sigma_{m}}\left(\epsilon \xi+z_{m}\right)=\sigma\left(\epsilon \xi+z_{m}\right), \text { for } \xi \in \partial B_{m}
\end{array}\right.
$$

and also

$$
\begin{array}{ll}
\frac{\partial \mathcal{U}^{m}(\xi)}{\partial \nu_{m}(\xi)} & :=\lambda\left(\operatorname{div}_{\xi} \mathcal{U}^{m}(\xi)\right) N_{m}(\xi)+\mu\left(\nabla_{\xi} \mathcal{U}^{m}(\xi)+\nabla_{\xi} \mathcal{U}^{m}(\xi)^{\top}\right) N_{m}(\xi) \\
= & \epsilon\left[\lambda\left(\operatorname{div} U^{\sigma_{m}}\left(\epsilon \xi+z_{m}\right)\right) N_{m}\left(\epsilon \xi+z_{m}\right)+\mu\left(\nabla U^{\sigma_{m}}\left(\epsilon \xi+z_{m}\right)+\nabla U^{\sigma_{m}}\left(\epsilon \xi+z_{m}\right)^{\top}\right) N_{m}\left(\epsilon \xi+z_{m}\right)\right] \\
= & \epsilon \frac{\partial U^{\sigma_{m}}}{\partial \nu_{m}}\left(\epsilon \xi+z_{m}\right) .
\end{array}
$$

Hence,

$$
\begin{aligned}
\left\|\frac{\partial \mathcal{U}^{m}}{\partial \nu_{m}}\right\|_{L^{2}\left(\partial B_{m}\right)}^{2} & =\int_{\partial B_{m}}\left|\frac{\partial \mathcal{U}^{m}(\eta)}{\partial \nu_{m}(\eta)}\right|^{2} d \eta \\
& =\int_{\partial D_{m}} \epsilon^{2}\left|\frac{\partial U^{\sigma_{m}}(s)}{\partial \nu_{m}(s)}\right|^{2} \epsilon^{-2} d s,\left[s:=\epsilon \eta+z_{m}\right] \\
& =\left\|\frac{\partial U^{\sigma_{m}}}{\partial \nu_{m}}\right\|_{L^{2}\left(\partial D_{m}\right)}^{2},
\end{aligned}
$$


which gives us

$$
\frac{\left\|\frac{\partial U^{\sigma_{m}}}{\partial \nu_{m}}\right\|_{L^{2}\left(\partial D_{m}\right)}}{\left\|U^{\sigma_{m}}\right\|_{H^{1}\left(\partial D_{m}\right)}} \underset{\mathbf{2 . 2 3}}{\leq} \frac{\left\|\frac{\partial \mathcal{U}^{m}}{\partial \nu_{m}}\right\|_{L^{2}\left(\partial B_{m}\right)}}{\epsilon\left\|\mathcal{U}^{m}\right\|_{H^{1}\left(\partial B_{m}\right)}} .
$$

For every function $\zeta_{m} \in H^{1}\left(\partial D_{m}\right)$, the corresponding $U^{\zeta_{m}}$ exists in $D_{m}$ as mentioned in (2.58) and then the corresponding functions $\mathcal{U}^{m}$ in $B_{m}$ and the inequality (2.64) will be satisfied by these functions. Let $\Lambda_{D_{m}}: H^{1}\left(\partial D_{m}\right) \rightarrow L^{2}\left(\partial D_{m}\right)$ and $\Lambda_{B_{m}}: H^{1}\left(\partial B_{m}\right) \rightarrow L^{2}\left(\partial B_{m}\right)$ be the Dirichlet to Neumann maps. Then we get the following estimate from (2.64).

$$
\left\|\Lambda_{D_{m}}\right\|_{\mathcal{L}\left(H^{1}\left(\partial D_{m}\right), L^{2}\left(\partial D_{m}\right)\right)} \leq \frac{1}{\epsilon}\left\|\Lambda_{B_{m}}\right\|_{\mathcal{L}\left(H^{1}\left(\partial B_{m}\right), L^{2}\left(\partial B_{m}\right)\right)} .
$$

This implies that,

$$
\begin{aligned}
\frac{\left\|\frac{\partial U^{\sigma_{m}}}{\partial \nu_{m}}\right\|_{H^{-1}\left(\partial D_{m}\right)}}{\left\|U^{\sigma_{m}}\right\|_{L^{2}\left(\partial D_{m}\right)}} & \leq\left\|\Lambda_{D_{m}}^{*}\right\|_{\mathcal{L}\left(L^{2}\left(\partial D_{m}\right), H^{-1}\left(\partial D_{m}\right)\right)} \\
& =\left\|\Lambda_{D_{m}}\right\|_{\mathcal{L}\left(H^{1}\left(\partial D_{m}\right), L^{2}\left(\partial D_{m}\right)\right)} \\
& \leq \frac{1}{\epsilon}\left\|\Lambda_{B_{m}}\right\|_{\mathcal{L}\left(H^{1}\left(\partial B_{m}\right), L^{2}\left(\partial B_{m}\right)\right)}
\end{aligned}
$$

Now, by (2.56) and (2.58),

$$
\left\|\frac{\partial U^{\sigma_{m}}}{\partial \nu_{m}}\right\|_{H^{-1}\left(\partial D_{m}\right)} \leq \grave{\mathcal{C}}(\omega)\left\|\Lambda_{B_{m}}\right\|_{\mathcal{L}\left(H^{1}\left(\partial B_{m}\right), L^{2}\left(\partial B_{m}\right)\right)} .
$$

Hence the result is true as $\left\|\Lambda_{B_{m}}\right\|_{\mathcal{L}\left(H^{1}\left(\partial B_{m}\right), L^{2}\left(\partial B_{m}\right)\right)}$ is bounded by a constant depending only on $B_{m}$ through its size and Lipschitz character of $B_{m}$.

Definition 2.9. We call $\sigma_{m} \in L^{2}\left(\partial D_{m}\right)$ satisfying (2.5), the solution of the problem (1.1] [1.3), as elastic surface charge distributions (in short surface charge distributions). Using these surface charge distributions we define the total charge on each surface $\partial D_{m}$ denoted by $Q_{m}$ as

$$
Q_{m}:=\int_{\partial D_{m}} \frac{\partial U^{\sigma_{m}}(s)}{\partial \nu_{m}(s)} d s
$$

\subsubsection{Estimates on the total charge $Q_{m}, m=1, \ldots M$}

In the following proposition, we provide an approximate of the far-fields in terms of the total charges $Q_{m}$.

Proposition 2.10. The P-part, $U_{p}^{\infty}(\hat{x}, \theta)$, and the $S$-part, $U_{s}^{\infty}(\hat{x}, \theta)$, of the far-field pattern of the problem (1.1] [1.3) have the following asymptotic expansions respectively;

$$
\begin{aligned}
U_{p}^{\infty}(\hat{x}, \theta) & =\frac{1}{4 \pi c_{p}^{2}}(\hat{x} \otimes \hat{x}) \sum_{m=1}^{M}\left[e^{-i \frac{\omega}{c_{p}} \hat{x} \cdot z_{m}} Q_{m}+O\left(a^{2}\right)\right], \\
U_{s}^{\infty}(\hat{x}, \theta) & =\frac{1}{4 \pi c_{s}^{2}}(I-\hat{x} \otimes \hat{x}) \sum_{m=1}^{M}\left[e^{-i \frac{\omega}{c_{s}} \hat{x} \cdot z_{m}} Q_{m}+O\left(a^{2}\right)\right] .
\end{aligned}
$$

if $\kappa_{p^{\omega}} a<1$ and $\kappa_{s^{\omega}} a<1$ where $O\left(a^{2}\right) \leq \grave{C}_{s p} \omega a^{2}$ with

$$
\grave{C}_{s p}:=\frac{(|\alpha|+|\beta|)|\partial B| \grave{\mathrm{C}}\left\|\Lambda_{B_{m}}\right\|_{\mathcal{L}\left(H^{1}\left(\partial B_{m}\right), L^{2}\left(\partial B_{m}\right)\right)}}{\max _{1 \leq m \leq M} \operatorname{diam}\left(B_{m}\right)} \frac{1}{\min \left\{c_{s}, c_{p}\right\}} .
$$


Proof. of Proposition 2.10, From (2.60), we have

$$
U^{s}(x)=\sum_{m=1}^{M} \int_{\partial D_{m}} \Gamma^{\omega}(x, s) \frac{\partial U^{\sigma_{m}}(s)}{\partial \nu_{m}(s)} d s, \text { for } x \in \mathbb{R}^{3} \backslash\left(\bigcup_{m=1}^{M} \bar{D}_{m}\right) .
$$

Substitution of the asymptotic behavior of the Kupradze tensor at infinity given in (2.2) in the above scattered field and comparing with (1.4), will allow us to write the P-part, $U_{p}^{\infty}(\hat{x}, \theta)$, and the $\mathrm{S}$-part, $U_{s}^{\infty}(\hat{x}, \theta)$, of the far-field pattern of the problem (1.1-1.3) respectively as;

$$
\begin{aligned}
U_{p}^{\infty}(\hat{x}, \theta) & =\frac{1}{4 \pi c_{p}^{2}}(\hat{x} \otimes \hat{x}) \sum_{m=1}^{M} \int_{S_{m}} e^{-i \kappa_{p} \omega \hat{x} \cdot s} \frac{\partial U^{\sigma_{m}}(s)}{\partial \nu_{m}(s)} d s \\
& =\frac{1}{4 \pi c_{p}^{2}}(\hat{x} \otimes \hat{x}) \sum_{m=1}^{M}\left[e^{-i \kappa_{p} \omega \hat{x} \cdot z_{m}} Q_{m}+\int_{S_{m}}\left[e^{-i \kappa_{p} \omega \hat{x} \cdot s}-e^{-i \kappa_{p} \omega \hat{x} \cdot z_{m}}\right] \frac{\partial U^{\sigma_{m}}(s)}{\partial \nu_{m}(s)} d s\right] \\
U_{p}^{\infty}(\hat{x}, \theta) & =\frac{1}{4 \pi c_{s}^{2}}(I-\hat{x} \otimes \hat{x}) \sum_{m=1}^{M} \int_{S_{m}} e^{-i \kappa_{s} \omega \hat{x} \cdot s} \frac{\partial U^{\sigma_{m}}(s)}{\partial \nu_{m}(s)} d s \\
& =\frac{1}{4 \pi c_{s}^{2}}(I-\hat{x} \otimes \hat{x}) \sum_{m=1}^{M}\left[e^{-i \kappa_{s} \omega \hat{x} \cdot z_{m}} Q_{m}+\int_{S_{m}}\left[e^{-i \kappa_{s} \omega \hat{x} \cdot s}-e^{-i \kappa_{s} \omega \hat{x} \cdot z_{m}}\right] \frac{\partial U^{\sigma_{m}}(s)}{\partial \nu_{m}(s)} d s\right] \cdot(2 .)
\end{aligned}
$$

For every $m=1,2, \ldots, M$, we have from Lemma 2.8

$$
\begin{aligned}
\left|\int_{\partial D_{m}}\right| \frac{\partial U^{\sigma_{m}}(s)}{\partial \nu_{m}(s)}|d s| & \leq\|1\|_{H^{1}\left(\partial D_{m}\right)} \cdot\left\|\frac{\partial U^{\sigma_{m}}}{\partial \nu_{m}}\right\|_{H^{-1}\left(\partial D_{m}\right)} \\
& \leq \epsilon \mid \partial \mathrm{B}^{\frac{1}{2}} \cdot\left\|\frac{\partial U^{\sigma_{m}}}{\partial \nu_{m}}\right\| \|_{H^{-1}\left(\partial D_{m}\right)}^{\leq} \\
& \grave{C} a
\end{aligned}
$$

with $\grave{C}:=\frac{\grave{\mathcal{C}}(\omega)|\partial \mathrm{B}|^{\frac{1}{2}}\left\|\Lambda_{B_{m}}\right\|_{\mathcal{L}\left(H^{1}\left(\partial B_{m}\right), L^{2}\left(\partial B_{m}\right)\right)}}{\max _{1 \leq m \leq M} \operatorname{diam}\left(B_{m}\right)}=\frac{(|\alpha|+|\beta|)|\partial \mathrm{B}| \grave{\mathrm{C}}\left\|\Lambda_{B_{m}}\right\|_{\mathcal{L}\left(H^{1}\left(\partial B_{m}\right), L^{2}\left(\partial B_{m}\right)\right)}}{\max _{1 \leq m \leq M} \operatorname{diam}\left(B_{m}\right)}$. It gives us the following estimate for any $\kappa$, i.e. $\kappa=\kappa_{p^{\omega}}$ or $\kappa_{s^{\omega}}$;

$$
\begin{aligned}
\left|\int_{\partial D_{m}}\left[e^{-i \kappa \hat{x} \cdot s}-e^{-i \kappa \hat{x} \cdot z_{m}}\right] \frac{\partial U^{\sigma_{m}}(s)}{\partial \nu_{m}(s)} d s\right| & \leq \int_{\partial D_{m}}\left|e^{-i \kappa \hat{x} \cdot s}-e^{-i \kappa \hat{x} \cdot z_{m}}\right|\left|\frac{\partial U^{\sigma_{m}}(s)}{\partial \nu_{m}(s)}\right| d s \\
& \leq \int_{\partial D_{m}} \sum_{l=1}^{\infty} \kappa^{l}\left|s-z_{m}\right|^{l}\left|\frac{\partial U^{\sigma_{m}}(s)}{\partial \nu_{m}(s)}\right| d s \\
& \leq \int_{\partial D_{m}} \sum_{l=1}^{\infty} \kappa^{l}\left(\frac{a}{2}\right)^{l}\left|\frac{\partial U^{\sigma_{m}}(s)}{\partial \nu_{m}(s)}\right| d s \\
\leq & \grave{C} a \sum_{l=1}^{\infty} \kappa^{l}\left(\frac{a}{2}\right)^{l}, \\
& =\frac{1}{2} \grave{C} \kappa a^{2} \frac{1}{1-\frac{1}{2} \kappa a}, \text { if } a<\frac{2}{\kappa_{\max }}\left(\leq \frac{2}{\kappa}\right) .
\end{aligned}
$$

which means

$$
\int_{\partial D_{m}}\left[e^{-i \kappa \hat{x} \cdot s}-e^{-i \kappa \hat{x} \cdot z_{m}}\right] \frac{\partial U^{\sigma_{m}}(s)}{\partial \nu_{m}(s)} d s \leq \grave{C} \kappa a^{2}, \text { for } a \leq \frac{1}{\kappa_{\max }}
$$


From (2.74), it follows that

$$
\begin{gathered}
\int_{S_{m}}\left[e^{-i \kappa_{p} \omega \hat{x} \cdot s}-e^{-i \kappa_{p} \omega \hat{x} \cdot z_{m}}\right] \frac{\partial U^{\sigma_{m}}(s)}{\partial \nu_{m}(s)} d s<\grave{C} \kappa_{p} \omega a^{2}, \text { if } \epsilon \leq \frac{\min \left\{c_{s}, c_{p}\right\}}{\omega_{\max } \max _{m} \operatorname{diam}\left(B_{m}\right)} \\
\int_{S_{m}}\left[e^{-i \kappa_{s} \omega \hat{x} \cdot s}-e^{-i \kappa_{s} \omega \hat{x} \cdot z_{m}}\right] \frac{\partial U^{\sigma_{m}}(s)}{\partial \nu_{m}(s)} d s<\kappa_{s}<\kappa_{s} a^{2}, \text { if } \epsilon \leq \frac{\min \left\{c_{s}, c_{p}\right\}}{\omega_{\max } \max _{m} \operatorname{diam}\left(B_{m}\right)} .
\end{gathered}
$$

Now substitution of (2.75) in (2.70) and (2.76) in (2.71) gives the required results (2.68), (2.69) respectively.

Lemma 2.11. For $m=1,2, \ldots, M$, the absolute value of the total charge $Q_{m}$ on each surface $\partial D_{m}$ is bounded by $\epsilon$, i.e.

$$
\left|Q_{m}\right| \leq \grave{\tilde{c}} \epsilon
$$

where $\grave{\tilde{c}}:=(|\alpha|+|\beta|)|\partial B| \grave{\mathrm{C}}\left\|\Lambda_{B_{m}}\right\|_{\mathcal{L}\left(H^{1}\left(\partial B_{m}\right), L^{2}\left(\partial B_{m}\right)\right)}$ with $\partial B$ and $\grave{\mathrm{C}}$ are defined in (2.44) and (2.54) respectively.

Proof. of Lemma 2.11. The proof follows as below;

$$
\begin{aligned}
\left|Q_{m}\right| & =\left|\int_{\partial D_{m}} \frac{\partial U^{\sigma_{m}}(s)}{\partial \nu_{m}(s)} d s\right| \\
& \leq\|1\|_{H^{1}\left(\partial D_{m}\right)}\left\|\frac{\partial U^{\sigma_{m}}(s)}{\partial \nu_{m}(s)}\right\|_{H^{-1}\left(\partial D_{m}\right)} \\
\frac{\leq}{2.66} & \|1\|_{L^{2}\left(\partial D_{m}\right)} \grave{\mathcal{C}}(\omega)\left\|\Lambda_{B_{m}}\right\|_{\mathcal{L}\left(H^{1}\left(\partial B_{m}\right), L^{2}\left(\partial B_{m}\right)\right)} \\
\underline{\underline{2.56}} & \epsilon|\partial \mathrm{B}|(|\alpha|+|\beta|) \grave{\mathrm{C}}\left\|\Lambda_{B_{m}}\right\|_{\mathcal{L}\left(H^{1}\left(\partial B_{m}\right), L^{2}\left(\partial B_{m}\right)\right)}
\end{aligned}
$$

For $s_{m} \in \partial D_{m}$, using the Dirichlet boundary condition (1.2), we have

$$
\begin{aligned}
0= & U^{t}\left(s_{m}\right)=U^{i}\left(s_{m}\right)+\sum_{j=1}^{M} \int_{\partial D_{j}} \Gamma^{\omega}\left(s_{m}, s\right) \frac{\partial U^{\sigma_{j}}(s)}{\partial \nu_{j}(s)} d s \\
= & U^{i}\left(s_{m}\right)+\sum_{\substack{j=1 \\
j \neq m}}^{M} \int_{\partial D_{j}} \Gamma^{\omega}\left(s_{m}, s\right) \frac{\partial U^{\sigma_{j}}(s)}{\partial \nu_{j}(s)} d s+\int_{\partial D_{m}} \Gamma^{\omega}\left(s_{m}, s\right) \frac{\partial U^{\sigma_{m}}(s)}{\partial \nu_{m}(s)} d s \\
= & U^{i}\left(s_{m}\right)+\sum_{\substack{j=1 \\
j \neq m}}^{M} \Gamma^{\omega}\left(s_{m}, z_{j}\right) Q_{j} \\
& \quad+\sum_{\substack{j=1 \\
j \neq m}}^{M}\left(\int_{\partial D_{j}}\left[\Gamma^{\omega}\left(s_{m}, s\right)-\Gamma^{\omega}\left(s_{m}, z_{j}\right)\right] \frac{\partial U^{\sigma_{j}}(s)}{\partial \nu_{j}(s)} d s\right)+\int_{\partial D_{m}} \Gamma^{\omega}\left(s_{m}, s\right) \frac{\partial U^{\sigma_{m}}(s)}{\partial \nu_{m}(s)} d s .
\end{aligned}
$$

To estimate $\int_{\partial D_{j}}\left[\Gamma^{\omega}\left(s_{m}, s\right)-\Gamma^{\omega}\left(s_{m}, z_{j}\right)\right] \frac{\partial U^{\sigma_{j}}(s)}{\partial \nu_{j}(s)}(s) d s$ for $j \neq m$, we have from Taylor series that,

$$
\Gamma^{\omega}\left(s_{m}, s\right)-\Gamma^{\omega}\left(s_{m}, z_{j}\right)=\left(s-z_{j}\right) \cdot R\left(s_{m}, s\right), R\left(s_{m}, s\right)=\int_{0}^{1} \nabla_{2} \Gamma^{\omega}\left(s_{m}, s-\alpha\left(s-z_{j}\right)\right) d \alpha .
$$

- From the definition of $\Gamma^{\omega}(x, y)$ and by using the calculations made in (2.49), for $s \in \bar{D}_{j}$, we obtain

$$
\left|R\left(s_{m}, s\right)\right| \leq \max _{y \in \bar{D}_{j}}\left|\nabla_{y} \Gamma^{\omega}\left(s_{m}, y\right)\right|<\frac{1}{4 \pi}\left[\frac{C_{9}}{d_{m j}^{2}}+C_{10}\right]
$$

with 
$C_{9}:=3\left(\frac{1}{c_{s}^{2}}+\frac{1}{c_{p}^{2}}\right)$
and $C_{10}:=2 \frac{\omega^{2}}{c_{s}^{4}}\left(\frac{1}{8}+\frac{1-\left(\frac{1}{2} \kappa_{s} \omega \operatorname{diam}(\Omega)\right)^{N_{\Omega}}}{1-\frac{1}{2} \kappa_{s} \omega \operatorname{diam}(\Omega)}+\frac{1}{2^{N_{\Omega}-1}}\right)+\frac{\omega^{2}}{c_{p}^{4}}\left(\frac{1}{4}+\frac{1-\left(\frac{1}{2} \kappa_{p} \omega \operatorname{diam}(\Omega)\right)^{N_{\Omega}}}{1-\frac{1}{2} \kappa_{p} \omega \operatorname{diam}(\Omega)}+\frac{1}{2^{N_{\Omega^{-1}}}}\right)$.

Indeed, for $x \in \bar{D}_{m}$ and $s \in \bar{D}_{j}$, we have from (2.4);

$$
\begin{aligned}
&\left|\nabla_{x} \Gamma^{\omega}(x, s)\right| \leq \frac{1}{4 \pi} \frac{1}{\omega^{2}}\left[3\left(\kappa_{s^{\omega}}^{2}+\kappa_{p^{\omega}}^{2}\right)|x-s|^{-2}+\frac{1}{8}\left(6 \kappa_{s^{\omega}}^{4}+4 \kappa_{p^{\omega}}^{4}\right)\right] \\
&+\frac{1}{4 \pi} \sum_{l=3}^{\infty} \frac{1}{(l-2) !(l+2)} \frac{1}{\omega^{2}}\left(2 \kappa_{s^{\omega}}^{l+2}+\kappa_{p^{\omega}}^{l+2}\right)|x-s|^{l-2} \\
& \leq \frac{1}{4 \pi} \frac{1}{\omega^{2}}\left[\frac{3}{d_{m j}^{2}}\left(\kappa_{s^{\omega}}^{2}+\kappa_{p^{\omega}}^{2}\right)+\frac{1}{4}\left(\kappa_{s^{\omega}}^{4}+\kappa_{p^{\omega}}^{4}\right)\right. \\
&\left.\quad+\sum_{l=2}^{\infty} \frac{1}{(l-2) !(l+2)}\left(2 \kappa_{s^{\omega}}^{l+2}+\kappa_{p^{\omega}}^{l+2}\right) \operatorname{diam}(\Omega)^{l-2}\right] \\
& \leq \frac{1}{4 \pi}\left[\frac{3}{d_{m j}^{2}}\left(\frac{1}{c_{s}^{2}}+\frac{1}{c_{p}^{2}}\right)+\frac{1}{4}\left(\frac{\omega^{2}}{c_{s}^{4}}+\frac{\omega^{2}}{c_{p}^{4}}\right)\right. \\
&\left.\quad+\sum_{l=2}^{\infty} \frac{1}{(l-2) !(l+2)}\left(2 \frac{\omega^{2}}{c_{s}^{4}} \kappa_{s^{\omega}}^{l-2}+\frac{\omega^{2}}{c_{p}^{4}} \kappa_{p^{\omega}}^{l-2}\right) \operatorname{diam}(\Omega)^{l-2}\right]
\end{aligned}
$$

[By recalling $N_{\Omega}=\left[2 \operatorname{diam}(\Omega) \max \left\{\kappa_{s^{\omega}}, \kappa_{p^{\omega}}\right\} e^{2}\right]$ and using Lemma 2.6]

$$
\begin{aligned}
& \leq \frac{1}{4 \pi}\left[\frac { 3 } { d _ { m j } ^ { 2 } } \left(\frac{1}{c_{s}^{2}}\right.\right.\left.+\frac{1}{c_{p}^{2}}\right)+\frac{1}{4}\left(\frac{\omega^{2}}{c_{s}^{4}}+\frac{\omega^{2}}{c_{p}^{4}}\right) \\
&+2 \frac{\omega^{2}}{c_{s}^{4}}\left(\sum_{l=0}^{N_{\Omega}-1}\left(\frac{1}{2} \kappa_{s} \omega \operatorname{diam}(\Omega)\right)^{l}+\sum_{l=N_{\Omega}}^{\infty}\left(\frac{1}{2}\right)^{l}\right) \\
&\left.+\frac{\omega^{2}}{c_{p}^{4}}\left(\sum_{l=0}^{N_{\Omega}-1}\left(\frac{1}{2} \kappa_{p^{\omega}} \operatorname{diam}(\Omega)\right)^{l}+\sum_{l=N_{\Omega}}^{\infty}\left(\frac{1}{2}\right)^{l}\right)\right] \\
&=\frac{1}{4 \pi}\left[\frac{C_{9}}{d_{m j}^{2}}+C_{10}\right] .
\end{aligned}
$$

For $m, j=1, \ldots, M$, and $j \neq m$, by making use of (2.80) and (2.72) we obtain the below;

$$
\begin{aligned}
\left|\int_{\partial D_{j}}\left[\Gamma^{\omega}\left(s_{m}, s\right)-\Gamma^{\omega}\left(s_{m}, z_{j}\right)\right] \frac{\partial U^{\sigma_{j}}(s)}{\partial \nu_{j}(s)} d s\right| & =\left|\int_{\partial D_{j}}\left(s-z_{j}\right) \cdot R\left(s_{m}, s\right) \frac{\partial U^{\sigma_{j}}(s)}{\partial \nu_{j}(s)} d s\right| \\
& \leq \int_{\partial D_{j}}\left|s-z_{j}\right|\left|R\left(s_{m}, s\right)\right| \frac{\partial U^{\sigma_{j}}(s)}{\partial \nu_{j}(s)} \mid d s \\
& <\frac{a}{4 \pi}\left[\frac{C_{9}}{d^{2}}+C_{10}\right] \int_{\partial D_{j}}\left|\frac{\partial U^{\sigma_{j}}(s)}{\partial \nu_{j}(s)}\right| d s \\
& <\grave{C} \frac{a}{4 \pi}\left[\frac{C_{9}}{d^{2}}+C_{10}\right] a .
\end{aligned}
$$


Then (2.78) can be written as

$$
\begin{aligned}
\int_{\partial D_{m}} \Gamma^{0}\left(s_{m}, s\right) \frac{\partial U^{\sigma_{m}}(s)}{\partial \nu_{m}(s)} d s+ & \int_{\partial D_{m}}\left[\Gamma^{\omega}\left(s_{m}, s\right)-\Gamma^{0}\left(s_{m}, s\right)\right] \frac{\partial U^{\sigma_{m}}(s)}{\partial \nu_{m}(s)} d s \\
& =-U^{i}\left(s_{m}\right)-\sum_{\substack{j=1 \\
j \neq m}}^{M} \Gamma^{\omega}\left(s_{m}, z_{j}\right) Q_{j}+O\left((M-1) \frac{a^{2}}{d^{2}}\right) .
\end{aligned}
$$

By using the Taylor series expansions of the exponential term $e^{i \kappa\left|s_{m}-s\right|}$, the above can also be written as,

$$
\int_{\partial D_{m}} \Gamma^{0}\left(s_{m}, s\right) \frac{\partial U^{\sigma_{m}}(s)}{\partial \nu_{m}(s)} d s+O(a)=-U^{i}\left(s_{m}\right)-\sum_{\substack{j=1 \\ j \neq m}}^{M} \Gamma^{\omega}\left(s_{m}, z_{j}\right) Q_{j}+O\left((M-1) \frac{a^{2}}{d^{2}}\right) .
$$

Indeed,

- $\omega \leq \omega_{\max }$ and for $m=1, \ldots, M$, we have

$$
\begin{aligned}
\mid \int_{\partial D_{m}} & {\left[\Gamma^{\omega}\left(s_{m}, s\right)-\Gamma^{0}\left(s_{m}, s\right)\right] \frac{\partial U^{\sigma_{m}}(s)}{\partial \nu_{m}(s)} d s \mid } \\
& \leq \int_{\partial D_{m}}\left|\Gamma^{\omega}\left(s_{m}, s\right)-\Gamma^{0}\left(s_{m}, s\right)\right|\left|\frac{\partial U^{\sigma_{m}}(s)}{\partial \nu_{m}(s)}\right| d s \\
& \leq \int_{\partial D_{m}} \frac{\omega}{4 \pi}\left[\frac{2}{c_{s}^{3}} \sum_{l=0}^{\infty}\left(\frac{1}{2}\right)^{l} \kappa_{s^{\omega}}^{l} \operatorname{diam}\left(D_{m}\right)^{l}+\frac{1}{c_{p}^{3}} \sum_{l=0}^{\infty}\left(\frac{1}{2}\right)^{l} \kappa_{p^{\omega}}^{l} \operatorname{diam}\left(D_{m}\right)^{l}\right]\left|\frac{\partial U^{\sigma_{m}}(s)}{\partial \nu_{m}(s)}\right| d s \\
& \quad \leq \frac{\omega}{(2.72)} \frac{2}{4 \pi}\left[\frac{2}{c_{s}^{3}} \sum_{l=0}^{\infty}\left(\frac{1}{2}\right)^{l} \kappa_{s^{\omega}}^{l} a^{l}+\frac{1}{c_{p}^{3}} \sum_{l=0}^{\infty}\left(\frac{1}{2}\right)^{l} \kappa_{p^{\omega}}^{l} a^{l}\right] \cdot \grave{C} a \\
& <\frac{\grave{C}}{\pi}\left[\frac{2}{c_{s}^{3}}+\frac{1}{c_{p}^{3}}\right] \omega a, \text { for } \epsilon \leq \frac{\left.\min _{\omega_{\max }} \max _{m}, c_{p}\right\}}{\operatorname{diam}\left(B_{m}\right)} .
\end{aligned}
$$

Define $U_{m}:=\int_{\partial D_{m}} \Gamma^{0}\left(s_{m}, s\right) \frac{\partial U^{\sigma_{m}}(s)}{\partial \nu_{m}(s)} d s, s_{m} \in \partial D_{m}$. Then (2.84) can be written as

$$
U_{m}=-U^{i}\left(s_{m}\right)-\sum_{\substack{j=1 \\ j \neq m}}^{M} \Gamma^{\omega}\left(s_{m}, z_{j}\right) Q_{j}+O(a)+O\left((M-1) \frac{a^{2}}{d^{2}}\right) .
$$

We set

$$
\bar{U}_{m}:=-U^{i}\left(z_{m}\right)-\sum_{\substack{j=1 \\ j \neq m}}^{M} \Gamma^{\omega}\left(z_{m}, z_{j}\right) Q_{j}, \text { for } m=1, \ldots, M .
$$

For $m=1, \ldots, M$, let $\bar{\sigma}_{m} \in L^{2}\left(\partial D_{m}\right)$ be the solutions of following the integral equation;

$$
\frac{\sigma_{m}(s)}{2}+\int_{\partial D_{m}} \frac{\partial \Gamma^{0}(x, s)}{\partial \nu_{m}(s)} \sigma_{m}(s) d s=\bar{U}_{m} \quad \text { on } \partial D_{m} .
$$

Remark here that the left hand side of (2.87) is the trace, on $\partial D_{m}$, of the double layer potential $\int_{\partial D_{m}} \frac{\partial \Gamma^{0}(x, s)}{\partial \nu_{m}(s)} \sigma_{m}(s) d s, x \in \mathbb{R}^{3} \backslash \bar{D}_{m}$. Dealing in the similar way as we derived (2.61), we obtain

$$
\int_{\partial D_{m}} \frac{\partial \Gamma^{0}(x, s)}{\partial \nu_{m}(s)} \sigma_{m}(s) d s=\int_{\partial D_{m}} \Gamma^{0}(x, s) \frac{\partial U^{\bar{\sigma}_{m}}(s)}{\partial \nu_{m}(s)} d s,
$$


with $U^{\bar{\sigma}_{m}}$ are the solutions of (2.58) replacing the frequency $\omega$ by zero. As single layer potential is continuotis up to the boundary, combining (2.87) and (2.88), we deduce that the constant potentials $\bar{U}_{m}, m=1, \ldots, M$ satisfy,

$$
\int_{\partial D_{m}} \Gamma^{0}\left(s_{m}, s\right) \frac{\partial U^{\bar{\sigma}_{m}}(s)}{\partial \nu_{m}(s)} d s=\bar{U}_{m}, s_{m} \in \partial D_{m} .
$$

The total charge on the surface $\partial D_{m}$ is given by

$$
\bar{Q}_{m}:=\int_{\partial D_{m}} \frac{\partial U^{\bar{\sigma}_{m}}(s)}{\partial \nu_{m}(s)} d s
$$

For $m=1, \ldots, M$, and $l=1,2,3$, [by proceeding in the similar manner as of (2.85)-(2.89)], let $\bar{\sigma}_{m}^{l} \in L^{2}\left(\partial D_{m}\right)$ be the surface charge distributions which define,

- The constant potentials $\bar{U}_{m}^{l} \in \mathbb{C}^{3 \times 1}$ as

$$
\int_{\partial D_{m}} \Gamma^{0}\left(s_{m}, s\right) \frac{\partial U^{\bar{\sigma}_{m}^{l}}(s)}{\partial \nu_{m}(s)} d s=\bar{U}_{m}^{l}:=-\left(U^{i}\left(z_{m}\right)\right)(l) e_{l}-\sum_{\substack{j=1 \\ j \neq m}}^{M} \Gamma^{\omega}\left(z_{m}, z_{j}\right) Q_{j}(l) e_{l}, s_{m} \in \partial D_{m}
$$

with $e_{1}=(1,0,0)^{\top}, e_{2}=(0,1,0)^{\top}$ and $e_{3}=(0,0,1)^{\top}$.

- The charge $\bar{Q}_{m}^{l} \in \mathbb{C}^{3 \times 1}$ on surface $S_{m}$ as

$$
\bar{Q}_{m}^{l}:=\int_{\partial D_{m}} \frac{\partial U^{\bar{\sigma}_{m}^{l}}(s)}{\partial \nu_{m}(s)} d s
$$

from which we can notice that $\bar{U}_{m}=\sum_{l=1}^{3} \bar{U}_{m}^{l}, \bar{\sigma}_{m}=\sum_{l=1}^{3} \bar{\sigma}_{m}^{l}$ and $\bar{Q}_{m}=\sum_{l=1}^{3} \bar{Q}_{m}^{l}$.

Now, we set the electrical capacitance $\bar{C}_{m} \in \mathbb{C}^{3 \times 3}$ for $1 \leq m \leq M$ through

$$
\bar{Q}_{m}^{l}=\bar{C}_{m} \bar{U}_{m}^{l}, l=1,2,3 \quad \text { and hence } \quad \bar{Q}_{m}=\bar{C}_{m} \bar{U}_{m} .
$$

We can write the above also as $\left[\bar{Q}_{m}^{1}, \bar{Q}_{m}^{2}, \bar{Q}_{m}^{3}\right]=\bar{C}_{m}\left[\bar{U}_{m}^{1}, \bar{U}_{m}^{2}, \bar{U}_{m}^{3}\right]$ for each $m=1,2, \ldots, M$.

Lemma 2.12. We have the following estimates for $1 \leq m \leq M$;

$$
\begin{aligned}
\left\|\frac{\partial U^{\sigma_{m}}}{\partial \nu_{m}}-\frac{\partial U^{\bar{\sigma}_{m}}}{\partial \nu_{m}}\right\|_{H^{-1}\left(\partial D_{m}\right)} & =O\left(a+(M-1) \frac{a^{2}}{d^{2}}\right), \\
Q_{m}-\bar{Q}_{m} & =O\left(a^{2}+(M-1) \frac{a^{3}}{d^{2}}\right) .
\end{aligned}
$$

where the constants appearing in $O(\cdot)$ depend only on the Lipschitz character of $B_{m}$.

Proof. of Lemma 2.12. By taking the difference between (2.85) and (2.89), we obtain

$$
\begin{aligned}
U_{m}-\bar{U}_{m} & =\int_{\partial D_{m}} \Gamma^{0}\left(s_{m}, s\right)\left(\frac{\partial U^{\sigma_{m}}}{\partial \nu_{m}}-\frac{\partial U^{\bar{\sigma}_{m}}}{\partial \nu_{m}}\right)(s) d s \\
& =O(a)+O\left((M-1) \frac{a^{2}}{d^{2}}\right), \quad s_{m} \in \partial D_{m} .
\end{aligned}
$$

Indeed, by using Taylor series,

- $U^{i}\left(s_{m}\right)-U^{i}\left(z_{m}\right)=O(a)$. 
- $\Gamma^{\omega}\left(s_{m}, z_{j}\right)-\Gamma^{\omega}\left(z_{m}, z_{j}\right)=O\left(\frac{a}{d^{2}}\right)$ and the asymptoticity of $Q_{j}$.

In operator form we can write (2.94) as,

$$
\begin{aligned}
\left(\mathcal{S}_{D_{m}}^{i_{\omega}}\right)^{*}\left(\frac{\partial U^{\sigma_{m}}}{\partial \nu_{m}}-\frac{\partial U^{\bar{\sigma}_{m}}}{\partial \nu_{m}}\right)\left(s_{m}\right) & :=\int_{\partial D_{m}} \Gamma^{0}\left(s_{m}, s\right)\left(\frac{\partial U^{\sigma_{m}}}{\partial \nu_{m}}-\frac{\partial U^{\bar{\sigma}_{m}}}{\partial \nu_{m}}\right)(s) d s \\
& =O(a)+O\left((M-1) \frac{a^{2}}{d^{2}}\right), \quad s_{m} \in \partial D_{m} .
\end{aligned}
$$

Here, $\left(\mathcal{S}_{D_{m}}^{i_{\omega}}\right)^{*}: H^{-1}\left(\partial D_{m}\right) \rightarrow L^{2}\left(\partial D_{m}\right)$ is the adjoint of $\mathcal{S}_{D_{m}}^{i_{\omega}}: L^{2}\left(\partial D_{m}\right) \rightarrow H^{1}\left(\partial D_{m}\right)$. We know that,

$$
\left\|\left(\mathcal{S}_{D_{m}}^{i_{\omega}}\right)^{*}\right\|_{\mathcal{L}\left(H^{-1}\left(\partial D_{m}\right), L^{2}\left(\partial D_{m}\right)\right)}=\left\|\mathcal{S}_{D_{m}}^{i_{\omega}}\right\|_{\mathcal{L}\left(L^{2}\left(\partial D_{m}\right), H^{1}\left(\partial D_{m}\right)\right)}
$$

and

$$
\left\|\left(\left(\mathcal{S}_{D_{m}}^{i_{\omega}}\right)^{*}\right)^{-1}\right\|_{\mathcal{L}\left(L^{2}\left(\partial D_{m}\right), H^{-1}\left(\partial D_{m}\right)\right)}=\left\|\left(\mathcal{S}_{D_{m}}^{i_{\omega}}\right)^{-1}\right\|_{\mathcal{L}\left(H^{1}\left(\partial D_{m}\right), L^{2}\left(\partial D_{m}\right)\right)}
$$

then from (4.4) of Lemma 4.2, we obtain $\left\|\left(\left(\mathcal{S}_{D_{m}}^{i_{\omega}}\right)^{*}\right)^{-1}\right\|_{\mathcal{L}\left(L^{2}\left(\partial D_{m}\right), H^{-1}\left(\partial D_{m}\right)\right)}=O\left(a^{-1}\right)$. Hence, we get the required results in the following manner.

- First,

$$
\begin{aligned}
\left\|\frac{\partial U^{\sigma_{m}}}{\partial \nu_{m}}-\frac{\partial U^{\bar{\sigma}_{m}}}{\partial \nu_{m}}\right\|_{H^{-1}\left(\partial D_{m}\right)} & \leq\left\|\left(\left(\mathcal{S}_{D_{m}}^{i_{\omega}}\right)^{*}\right)^{-1}\right\|_{\mathcal{L}\left(L^{2}\left(\partial D_{m}\right), H^{-1}\left(\partial D_{m}\right)\right)}\left\|O(a)+O\left((M-1) \frac{a^{2}}{d^{2}}\right)\right\|_{L^{2}\left(\partial D_{m}\right)} \\
& =O\left(a+(M-1) \frac{a^{2}}{d^{2}}\right) .
\end{aligned}
$$

- Second,

$$
\begin{aligned}
\left|Q_{m}-\bar{Q}_{m}\right| & =\left|\int_{\partial D_{m}}\left(\frac{\partial U^{\sigma_{m}}}{\partial \nu_{m}}-\frac{\partial U^{\bar{\sigma}_{m}}}{\partial \nu_{m}}\right)(s) d s\right| \\
& \leq\left\|\frac{\partial U^{\sigma_{m}}}{\partial \nu_{m}}-\frac{\partial U^{\bar{\sigma}_{m}}}{\partial \nu_{m}}\right\|_{H^{-1}\left(\partial D_{m}\right)}\|1\|_{H^{1}\left(\partial D_{m}\right)} \\
& =O\left(a^{2}+(M-1) \frac{a^{3}}{d^{2}}\right) .
\end{aligned}
$$

Lemma 2.13. For every $1 \leq m \leq M$, the capacitance $\bar{C}_{m}$ and charge $\bar{Q}_{m}$ are of the form;

$$
\bar{C}_{m}=\frac{\bar{C}_{B_{m}}}{\max _{1 \leq m \leq M} \operatorname{diam}\left(B_{m}\right)} a \quad \text { and } \quad \bar{Q}_{m}=\frac{\bar{Q}_{B_{m}}}{\max _{1 \leq m \leq M} \operatorname{diam}\left(B_{m}\right)} a
$$

where $\bar{C}_{B_{m}}$ and $\bar{Q}_{B_{m}}$ are the capacitance and the charge of $B_{m}$ respectively.

Proof. of Lemma 2.13. Take $0<\epsilon \leq 1, z \in \mathbb{R}^{3}$ and write, $D_{\epsilon}:=\epsilon B+z \subset \mathbb{R}^{3}$. For $\psi_{\epsilon} \in L^{2}\left(\partial D_{\epsilon}\right)$ and $\psi \in L^{2}(\partial B)$, define the operators $\mathcal{S}^{i_{\omega}}: L^{2}\left(\partial D_{\epsilon}\right) \rightarrow H^{1}\left(\partial D_{\epsilon}\right)$ and $\mathcal{S}_{B}^{i_{\omega}}: L^{2}(\partial B) \rightarrow H^{1}(\partial B)$ as;

$$
\mathcal{S}^{i_{\omega}} \psi_{\epsilon}(x):=\int_{\partial D_{\epsilon}} \Gamma^{0}(x, y) \psi_{\epsilon}(y) d y, \quad \text { and } \quad \mathcal{S}_{B}^{i_{\omega}} \psi(\xi):=\int_{\partial B} \Gamma^{0}(\xi, \eta) \psi(\eta) d \eta .
$$


Define $U^{\psi_{\epsilon}}$ and $U^{\psi}$ as the functions on $\bar{D}_{\epsilon}$ and $\bar{B}$ respectively in the similar way of (2.58). Then the operators

$$
\underline{\mathcal{S}}^{i_{\omega}} U^{\psi_{\epsilon}}(x):=\int_{\partial D_{\epsilon}} \Gamma^{0}(x, y) \frac{\partial U^{\psi_{\epsilon}}}{\partial \nu_{y}}(y) d y, \quad \text { and } \quad \underline{\mathcal{S}}_{B}^{i_{\omega}} U^{\psi}(\xi):=\int_{\partial B} \Gamma^{0}(\xi, \eta) \frac{\partial U^{\psi}}{\partial \nu_{\eta}}(\eta) d \eta .
$$

define the corresponding potentials $\bar{U}_{\epsilon}, \bar{U}_{B}$ on the surfaces $\partial D_{\epsilon}$ and $\partial B$ w.r.t the surface charge distributions $\psi_{\epsilon}$ and $\psi$ respectively. Let, these potentials be equal to some constant vector $D \in \mathbb{C}^{3 \times 1}$. Let the total charge of these conductors $D_{\epsilon}, B$ are $\bar{Q}_{\epsilon}$ and $\bar{Q}_{B}$, and the capacitances are $\bar{C}_{\epsilon}$ and $\bar{C}_{B}$ respectively. Then we can write these as,

$$
\bar{U}_{\epsilon}:=\underline{\mathcal{S}}^{i_{\omega}} U^{\psi_{\epsilon}}(x)=D, \quad \bar{U}_{B}:=\underline{\mathcal{S}}_{B}^{i_{\omega}} U^{\psi}(\xi)=D, \forall x \in \partial D_{\epsilon}, \forall \xi \in \partial B .
$$

We have by definitions, $\bar{Q}_{\epsilon}=\int_{\partial D_{\epsilon}} \frac{\partial U^{\psi_{\epsilon}}}{\partial \nu_{y}}(y) d y, \bar{Q}_{B}=\int_{\partial B} \frac{\partial U^{\psi}}{\partial \nu_{\eta}}(\eta) d \eta$, and $\bar{C}_{\epsilon} \bar{U}_{\epsilon}=\bar{Q}_{\epsilon}, \bar{C}_{B} \bar{U}_{B}=\bar{Q}_{B}$. Observe that,

$$
\begin{aligned}
D & =\underline{\mathcal{S}}^{i_{\omega}} U^{\psi_{\epsilon}}(x) & D & =\underline{\mathcal{S}}_{B}^{i_{\omega}} U^{\psi}(\xi) \\
& =\int_{\partial D_{\epsilon}} \Gamma^{0}(x, y) \frac{\partial U^{\psi_{\epsilon}}}{\partial \nu_{y}}(y) d y & & \int_{\partial B} \Gamma^{0}(\xi, \eta) \frac{\partial U^{\psi}}{\partial \nu_{\eta}}(\eta) d \eta \\
& =\int_{\partial B} \frac{1}{\epsilon} \Gamma^{0}(\xi, \eta) \frac{1}{\epsilon} \frac{\partial U^{\psi_{\epsilon}}}{\partial \nu_{\eta}}(\epsilon \eta+z) \epsilon^{2} d \eta & & \int_{\partial D_{\epsilon}} \epsilon \Gamma^{0}(x, y) \epsilon \frac{\partial U^{\psi}}{\partial \nu_{y}}(y-z / \epsilon) \epsilon^{-2} d y \\
& =\int_{\partial B} \Gamma^{0}(\xi, \eta) \frac{\partial \hat{U} \psi_{\epsilon}}{\partial \nu_{\eta}}(\eta) d \eta & & \int_{\partial D_{\epsilon}} \Gamma^{0}(x, y) \frac{\partial \check{U}^{\psi}}{\partial \nu_{y}}(y) d y \\
& =\underline{\mathcal{S}}_{B}^{i_{\omega}} \hat{U}^{\psi_{\epsilon}}(\xi) . \quad\left[\hat{\psi}_{\epsilon}(\eta):=\psi_{\epsilon}(\epsilon \eta+z)\right] & & =\underline{\mathcal{S}}^{i_{\omega}} \check{U}^{\psi}(x) . \quad\left[\check{\psi}(y):=\psi\left(\frac{y-z}{\epsilon}\right)\right]
\end{aligned}
$$

Hence, $U^{\psi_{\epsilon}}=\check{U}^{\psi}$ and $U^{\psi}=\hat{U}^{\psi_{\epsilon}}$. Now we have,

$$
\begin{aligned}
\bar{Q}_{\epsilon} & =\int_{\partial D_{\epsilon}} \frac{\partial U^{\psi_{\epsilon}}}{\partial \nu_{y}}(y) d y=\int_{\partial D_{\epsilon}} \frac{\partial \check{U}^{\psi}}{\partial \nu_{y}}(y) d y \\
& =\int_{\partial B} \frac{1}{\epsilon} \frac{\partial \check{U}^{\psi}}{\partial \nu_{\eta}}(\epsilon \eta+z) \epsilon^{2} d \eta=\epsilon \int_{\partial B} \frac{\partial \check{U}^{\psi}}{\partial \nu_{\eta}}(\epsilon \eta+z) d \eta \\
& =\epsilon \int_{\partial B} \frac{\partial \hat{\tilde{U}}^{\psi}}{\partial \nu_{\eta}}(\eta) d \eta=\epsilon \int_{\partial B} \frac{\partial U^{\psi}}{\partial \nu_{\eta}}(\eta) d \eta \\
& =\epsilon \bar{Q}_{B}
\end{aligned}
$$

which gives us,

$$
\bar{C}_{\epsilon} D=\bar{C}_{\epsilon} \bar{U}_{\epsilon}=\bar{Q}_{\epsilon}=\epsilon \bar{Q}_{B}=\epsilon \bar{C}_{B} \bar{U}_{B}=\epsilon \bar{C}_{B} D
$$

It is true for every constant vector $D$ and hence $\bar{C}_{\epsilon}=\epsilon \bar{C}_{B}$. As we have $D_{m}=\epsilon B_{m}+z_{m}$ and $a=$ $\max _{1 \leq m \leq M} \operatorname{diam} D_{m}=\epsilon \max _{1 \leq m \leq M} \operatorname{diam}\left(B_{m}\right)$, we obtain

$$
\bar{Q}_{m}=\epsilon \bar{Q}_{B_{m}}=\frac{\bar{Q}_{B_{m}}}{\max _{1 \leq m \leq M} \operatorname{diam}\left(B_{m}\right)} a \quad \text { and } \quad \bar{C}_{m}=\epsilon \bar{C}_{B_{m}}=\frac{\bar{C}_{B_{m}}}{\max _{1 \leq m \leq M} \operatorname{diam}\left(B_{m}\right)} a .
$$

Lemma 2.14. For $m=1,2, \ldots, M$, the elastic capacitances $\bar{C}_{m} \in \mathbb{C}^{3 \times 3}$ defined through (2.91) are nonsingular. 
Proof. of Proposition 2.14. As the capacitances $\bar{C}_{m}$ depend only on the scatterers, let $\sigma_{m}^{l} \in L^{2}\left(\partial D_{m}\right)$ Be surface charge distributions which define the potentials $e_{l}$ for $l=1,2,3$. i.e.

$$
\int_{\partial D_{m}} \Gamma^{0}\left(s_{m}, s\right) \frac{\partial U^{\sigma_{m}} l}{\partial \nu_{m}}(s) d s=e_{l}=: U_{m}^{l}, \text { for } l=1,2,3, m=1, \ldots, M .
$$

We also have $\left[\int_{\partial D_{m}} \frac{\partial U^{\sigma_{m}}}{\partial \nu_{m}}{ }^{1}(s) d s, \int_{\partial D_{m}} \frac{\partial U^{\sigma_{m}}}{\partial \nu_{m}}(s) d s, \int_{\partial D_{m}} \frac{\partial U^{\sigma_{m}}}{\partial \nu_{m}}(s) d s\right]=\bar{C}_{m}\left[U_{m}^{1}, U_{m}^{2}, U_{m}^{3}\right]=\bar{C}_{m}$. Hence, it is enough if we show that the matrix $\left[\int_{\partial D_{m}}\left(\frac{\partial U^{\sigma_{m}} l}{\partial \nu_{m}}\right)_{j}(s) d s\right]_{l, j=1}^{3}$ is invertible. In order to prove this, assume the linear combination $\sum_{l=1}^{3} a_{l} \int_{\partial D_{m}} \frac{\partial U^{\sigma_{m}} l}{\partial \nu_{m}}(s) d s=0$ for the scalars $a_{l} \in \mathbb{C}$. From (2.96), we can deduce that

$$
\int_{\partial D_{m}} \int_{\partial D_{m}} \Gamma^{0}\left(s_{m_{1}}, s_{m_{2}}\right)\left(\sum_{l=1}^{3} a_{l} \frac{\partial U^{\sigma_{m}} l}{\partial \nu_{m}}\left(s_{m_{2}}\right)\right) \cdot \frac{\partial U^{\sigma_{m}} j}{\partial \nu_{m}}\left(s_{m_{1}}\right) d s_{m_{1}} d s_{m_{2}}=0, j=1,2,3
$$

and hence

$$
\int_{\partial D_{m}} \int_{\partial D_{m}} \Gamma^{0}\left(s_{m_{1}}, s_{m_{2}}\right)\left(\sum_{l=1}^{3} a_{l} \frac{\partial U^{\sigma_{m}} l}{\partial \nu_{m}}\left(s_{m_{2}}\right)\right) \cdot\left(\sum_{j=1}^{3} a_{j} \frac{\partial U^{\sigma_{m}} j}{\partial \nu_{m}}\left(s_{m_{1}}\right)\right) d s_{m_{1}} d s_{m_{2}}=0 .
$$

The positivity of the single layer operator implies, $\sum_{l=1}^{3} a_{l} \frac{\partial U^{\sigma_{m}} l}{\partial \nu_{m}}(s)=0, s \in \partial D_{m}$.

Again now by making use of (2.96), we deduce

$$
\sum_{l=1}^{3} a_{l} e_{l}=\int_{\partial D_{m}} \Gamma^{0}\left(s_{m}, s\right)\left(\sum_{l=1}^{3} a_{l} \frac{\partial U^{\sigma_{m}} l}{\partial \nu_{m}}(s)\right) d s=0, s_{m} \in \partial D_{m}
$$

and hence $a_{l}=0$ for $l=1,2,3$.

Proposition 2.15. For $m=1,2, \ldots, M$, the total charge $\bar{Q}_{m}$ on each surface $\partial D_{m}$ of the small scatterer $D_{m}$ can be calculated from the algebraic system

$$
\bar{C}_{m}^{-1} \bar{Q}_{m}=-U^{i}\left(z_{m}\right)-\sum_{\substack{j=1 \\ j \neq m}}^{M} \Gamma^{\omega}\left(z_{m}, z_{j}\right) \bar{C}_{j}\left(\bar{C}_{j}^{-1} \bar{Q}_{j}\right)
$$

with an error of order $O\left((M-1) \frac{a^{2}}{d}+(M-1)^{2} \frac{a^{3}}{d^{3}}\right)$.

Proof. of Proposition 2.15, We can rewrite (2.89) as

$$
\begin{aligned}
\bar{C}_{m}^{-1} \bar{Q}_{m} & =-U^{i}\left(z_{m}\right)-\sum_{\substack{j=1 \\
j \neq m}}^{M} \Gamma^{\omega}\left(z_{m}, z_{j}\right) Q_{j} \\
& =-U^{i}\left(z_{m}\right)-\sum_{\substack{j=1 \\
j \neq m}}^{M} \Gamma^{\omega}\left(z_{m}, z_{j}\right) \bar{Q}_{j}-\sum_{\substack{j=1 \\
j \neq m}}^{M} \Gamma^{\omega}\left(z_{m}, z_{j}\right)\left(Q_{j}-\bar{Q}_{j}\right) \\
& =-U^{i}\left(z_{m}\right)-\sum_{\substack{j=1 \\
j \neq m}}^{M} \Gamma^{\omega}\left(z_{m}, z_{j}\right) \bar{Q}_{j}+O\left((M-1) \frac{a^{2}}{d}+(M-1)^{2} \frac{a^{3}}{d^{3}}\right),
\end{aligned}
$$


where we used (2.93) and the fact $\Gamma^{\omega}\left(z_{m}, z_{j}\right)=O\left(\frac{1}{d}+\omega\right), \omega \leq \omega_{\max }$ and $d \leq d_{\max }$. Indeed,

$$
\begin{aligned}
\left|\Gamma^{\omega}\left(z_{m}, z_{j}\right)\right| & \leq \frac{1}{4 \pi} \frac{1}{\omega^{2}}\left(\kappa_{s^{\omega}}^{2}+\kappa_{p^{\omega}}^{2}\right)\left|z_{m}-z_{j}\right|^{-1}+\frac{1}{4 \pi} \sum_{l=1}^{\infty} \frac{1}{(l-1) !(l+2)} \frac{1}{\omega^{2}}\left(2 \kappa_{s^{\omega}}^{l+2}+\kappa_{p^{\omega}}^{l+2}\right)\left|z_{m}-z_{j}\right|^{l-1} \\
& \leq \frac{1}{4 \pi} \frac{1}{\omega^{2}}\left[\frac{1}{d_{m j}}\left(\kappa_{s^{\omega}}^{2}+\kappa_{p^{\omega}}^{2}\right)+\sum_{l=1}^{\infty} \frac{1}{(l-1) !(l+2)}\left(2 \kappa_{s^{\omega}}^{l+2}+\kappa_{p^{\omega}}^{l+2}\right) \operatorname{diam}(\Omega)^{l-1}\right] \\
& \leq \frac{1}{4 \pi}\left[\frac{1}{d_{m j}}\left(\frac{1}{c_{s}^{2}}+\frac{1}{c_{p}^{2}}\right)+\sum_{l=1}^{\infty} \frac{1}{(l-1) !(l+2)}\left(2 \frac{\omega}{c_{s}^{3}} \kappa_{s^{\omega}}^{l-1}+\frac{\omega}{c_{p}^{3}} \kappa_{p^{\omega}}^{l-1}\right) \operatorname{diam}(\Omega)^{l-1}\right]
\end{aligned}
$$

[By recalling $N_{\Omega}=\left[2 \operatorname{diam}(\Omega) \max \left\{\kappa_{s} \omega, \kappa_{p^{\omega}}\right\} e^{2}\right]$ and using Lemma 2.6]

$$
\begin{aligned}
\leq \frac{1}{4 \pi}\left[\frac{1}{d_{m j}}\left(\frac{1}{c_{s}^{2}}+\frac{1}{c_{p}^{2}}\right)+2 \frac{\omega}{c_{s}^{3}}\left(\sum_{l=1}^{N_{\Omega}}\left(\frac{1}{2} \kappa_{s \omega} \operatorname{diam}(\Omega)\right)^{l-1}+\sum_{l=N_{\Omega}+1}^{\infty} \frac{1}{2^{l-1}}\right)\right. \\
\left.+\frac{\omega}{c_{p}^{3}}\left(\sum_{l=1}^{N_{\Omega}}\left(\frac{1}{2} \kappa_{p^{\omega}} \operatorname{diam}(\Omega)\right)^{l-1}+\sum_{l=N_{\Omega}+1}^{\infty} \frac{1}{2^{l-1}}\right)\right] \\
=\frac{1}{4 \pi}\left[\frac{1}{d_{m j}}\left(\frac{1}{c_{s}^{2}}+\frac{1}{c_{p}^{2}}\right)+2 \frac{\kappa_{s} \omega}{c_{s}^{2}}\left(\frac{1-\left(\frac{1}{2} \kappa_{s} \omega \operatorname{diam}(\Omega)\right)^{N_{\Omega}}}{1-\frac{1}{2} \kappa_{s} \omega \operatorname{diam}(\Omega)}+\frac{1}{2^{N_{\Omega}-1}}\right)\right. \\
\left.+\frac{\kappa_{p^{\omega}}}{c_{p}^{2}}\left(\frac{1-\left(\frac{1}{2} \kappa_{p^{\omega}} \operatorname{diam}(\Omega)\right)^{N_{\Omega}}}{1-\frac{1}{2} \kappa_{p^{\omega}} \operatorname{diam}(\Omega)}+\frac{1}{2^{N_{\Omega}-1}}\right)\right] \\
=\frac{1}{4 \pi}\left[\frac{C_{7}}{d_{m j}}+C_{8}\right]
\end{aligned}
$$

with

$$
C_{7}:=\left[\frac{1}{c_{s}^{2}}+\frac{2}{c_{p}^{2}}\right]
$$

and

$$
C_{8}:=2 \frac{\kappa_{s^{\omega}}}{c_{s}^{2}}\left(\frac{1-\left(\frac{1}{2} \kappa_{s^{\omega}} \operatorname{diam}(\Omega)\right)^{N_{\Omega}}}{1-\frac{1}{2} \kappa_{s^{\omega}} \operatorname{diam}(\Omega)}+\frac{1}{2^{N_{\Omega}-1}}\right)+\frac{\kappa_{p^{\omega}}}{c_{p}^{2}}\left(\frac{1-\left(\frac{1}{2} \kappa_{p^{\omega}} \operatorname{diam}(\Omega)\right)^{N_{\Omega}}}{1-\frac{1}{2} \kappa_{p^{\omega}} \operatorname{diam}(\Omega)}+\frac{1}{2^{N_{\Omega}-1}}\right) .
$$

\subsubsection{The algebraic system}

Define the algebraic system,

$$
\bar{C}_{m}^{-1} \tilde{Q}_{m}:=-U^{i}\left(z_{m}\right)-\sum_{\substack{j=1 \\ j \neq m}}^{M} \Gamma^{\omega}\left(z_{m}, z_{j}\right) \bar{C}_{j}\left(\bar{C}_{j}^{-1} \tilde{Q}_{j}\right)
$$

for all $m=1,2, \ldots, M$. It can be written in a compact form as

$$
\mathbf{B} \tilde{Q}=U^{I},
$$

where $\tilde{Q}, U^{I} \in \mathbb{C}^{3 M \times 1}$ and $\mathbf{B} \in \mathbb{C}^{3 M \times 3 M}$ are defined as

$$
\mathbf{B}:=\left(\begin{array}{ccccc}
-\bar{C}_{1}^{-1} & -\Gamma^{\omega}\left(z_{1}, z_{2}\right) & -\Gamma^{\omega}\left(z_{1}, z_{3}\right) & \ldots & -\Gamma^{\omega}\left(z_{1}, z_{M}\right) \\
-\Gamma^{\omega}\left(z_{2}, z_{1}\right) & -\bar{C}_{2}^{-1} & -\Gamma^{\omega}\left(z_{2}, z_{3}\right) & \ldots & -\Gamma^{\omega}\left(z_{2}, z_{M}\right) \\
\cdots & \ldots & \ldots & \ldots & \ldots \\
-\Gamma^{\omega}\left(z_{M}, z_{1}\right) & -\Gamma^{\omega}\left(z_{M}, z_{2}\right) & \ldots & -\Gamma^{\omega}\left(z_{M}, z_{M-1}\right) & -\bar{C}_{M}^{-1}
\end{array}\right),
$$




$$
\tilde{Q}:=\left(\begin{array}{cccc}
\tilde{Q}_{1}^{\top} & \tilde{Q}_{2}^{\top} & \ldots & \tilde{Q}_{M}^{\top}
\end{array}\right)^{\top} \text { and } U^{I}:=\left(\begin{array}{llll}
U^{i}\left(z_{1}\right)^{\top} & U^{i}\left(z_{2}\right)^{\top} & \ldots & U^{i}\left(z_{M}\right)^{\top}
\end{array}\right)^{\top} .
$$

The above linear algebraic system is solvable for the $3 \mathrm{D}$ vectors $\tilde{Q}_{j}, 1 \leq j \leq M$, when the matrix $\mathbf{B}$ is invertible. We discuss its invertibility in Section 3 ,

Now, the difference between (2.97) and (2.99) produce the following

$$
\bar{C}_{m}^{-1}\left(\bar{Q}_{m}-\tilde{Q}_{m}\right)=-\sum_{\substack{j=1 \\ j \neq m}}^{M} \Gamma^{\omega}\left(z_{m}, z_{j}\right)\left(\bar{Q}_{j}-\tilde{Q}_{j}\right)+O\left((M-1) \frac{a^{2}}{d}+(M-1)^{2} \frac{a^{3}}{d^{3}}\right) .
$$

for $m=1,2, \ldots, M$. Considering the above system of equations (2.101) in the place of (2.99) and then by making use of the Corollary 3.3 and the fact that acoustic capacitances of the sactterers are bounded above and below by their diameters multiplied by constants which depend only on the Lipschitz character of $B_{m}$ 's, see [11, Lemma 2.11 and Remark 2.23], we obtain

$$
\sum_{m=1}^{M}\left(\bar{Q}_{m}-\tilde{Q}_{m}\right)=O\left(M(M-1) \frac{a^{3}}{d}+M(M-1)^{2} \frac{a^{4}}{d^{3}}\right) .
$$

\subsection{End of the proof of Theorem 1.2}

The use of (2.93), (2.102) in (2.68) and (2.69) allows us to represent the asymptotic expansions of the P part, $U_{p}^{\infty}(\hat{x}, \theta)$, and the S part, $U_{p}^{\infty}(\hat{x}, \theta)$, of the far-field pattern of the problem (1.1] 1.3) in terms of $\tilde{Q}_{m}$ respectively as below;

$$
\begin{aligned}
U_{p}^{\infty}(\hat{x}, \theta) & =\frac{1}{4 \pi c_{p}^{2}}(\hat{x} \otimes \hat{x}) \sum_{m=1}^{M} e^{-i \frac{\omega}{c_{p}} \hat{x} \cdot z_{m}}\left[Q_{m}+O\left(a^{2}\right)\right] \\
& =\frac{1}{4 \pi c_{p}^{2}}(\hat{x} \otimes \hat{x}) \sum_{m=1}^{M} e^{-i \frac{\omega}{c_{p}} \hat{x} \cdot z_{m}}\left[\left[\tilde{Q}_{m}+\left(Q_{m}-\bar{Q}_{m}\right)+\left(\bar{Q}_{m}-\tilde{Q}_{m}\right)\right]+O\left(a^{2}\right)\right] \\
& =\frac{1}{4 \pi c_{p}^{2}}(\hat{x} \otimes \hat{x})\left(\sum_{m=1}^{M} e^{-i \frac{\omega}{c_{p}} \hat{x} \cdot z_{m}}\left[\tilde{Q}_{m}+O\left(a^{2}+(M-1) \frac{a^{3}}{d^{2}}\right)\right]+\sum_{m=1}^{M} e^{-i \frac{\omega}{c_{p}} \hat{x} \cdot z_{m}}\left(\bar{Q}_{m}-\tilde{Q}_{m}\right)\right) \\
& =\frac{1}{4 \pi c_{p}^{2}}(\hat{x} \otimes \hat{x})\left[\sum_{m=1}^{M} e^{-i \frac{\omega}{c_{p}} \hat{x} \cdot z_{m}} \tilde{Q}_{m}+O\left(M a^{2}+M(M-1) \frac{a^{3}}{d^{2}}+M(M-1)^{2} \frac{a^{4}}{d^{3}}\right)\right], \\
U_{s}^{\infty}(\hat{x}, \theta) & =\frac{1}{4 \pi c_{s}^{2}}(I-\hat{x} \otimes \hat{x}) \sum_{m=1}^{M} e^{-i \frac{\omega}{c_{s}} \hat{x} \cdot z_{m}}\left[Q_{m}+O\left(a^{2}\right)\right] \\
& =\frac{1}{4 \pi c_{s}^{2}}(I-\hat{x} \otimes \hat{x})\left[\sum_{m=1}^{M} e^{-i \frac{\omega}{c_{s}} \hat{x} \cdot z_{m}} \tilde{Q}_{m}+O\left(M a^{2}+M(M-1) \frac{a^{3}}{d^{2}}+M(M-1)^{2} \frac{a^{4}}{d^{3}}\right)\right](2.104)
\end{aligned}
$$

Hence, Theorem 1.2 is proved by setting $\bar{\sigma}_{m}:=\frac{\bar{\sigma}_{m}}{U_{m}}$ as the surface density which defines $\tilde{Q}_{m}$. Finally, let us remark that

1. The constant $\grave{c}:=\left[\frac{1}{4 \pi}\left[\tilde{C}_{7}+\tilde{C}_{8} d_{\max }^{2}\right]|\partial \mathrm{B}| \max _{m=1}^{M} \grave{C}_{6 m}\right]^{-\frac{1}{2}}$ appearing in Proposition 2.2 will serve our purpose in Theorem 1.2 by defining $c_{0}:=\grave{c} \max _{1 \leq m \leq M} \operatorname{diam}\left(B_{m}\right)$ respectively.

2. The coefficients $\bar{\sigma}_{m} \bar{U}_{m}^{-1}, \tilde{Q}_{m}, \bar{C}_{m}$ plays the roles of $\sigma_{m}, Q_{m}, C_{m}$ respectively in Theorem 1.2 , 
3. The constant appearing in $O\left(M a^{2}+M(M-1) \frac{a^{3}}{d^{2}}+M(M-1)^{2} \frac{a^{4}}{d^{3}}\right)$ is

$$
C^{e} \max \left\{1+\frac{\max _{1 \leq m \leq M} \bar{C}_{B_{m}}}{\max _{1 \leq m \leq M} \operatorname{diam}\left(B_{m}\right)} \frac{C_{7}+C_{8} d_{\max }}{4 \pi}, 1+\frac{\grave{C} \omega}{C^{e} \min \left\{c_{s}, c_{p}\right\}}\right\}
$$

with $\quad C^{e} \quad:=\quad \frac{\max _{1 \leq m \leq M}\left\|\mathcal{S}_{B_{m}}^{i_{\omega}}{ }^{-1}\right\|_{\mathcal{L}\left(H^{1}\left(\partial B_{m}\right), L^{2}\left(\partial B_{m}\right)\right)}|\partial \mathrm{B}|}{\max _{1 \leq m \leq M} \operatorname{diam}\left(B_{m}\right)} \max \left\{\left(\frac{|\alpha|+|\beta|}{\min \left\{c_{s}, c_{p}\right\}}+\frac{\grave{C}}{\pi}\left[\frac{2}{c_{s}^{3}}+\frac{1}{c_{p}^{3}}\right]\right) \omega\right.$,

$\left.\frac{\grave{C}}{4 \pi}\left[C_{9}+C_{10} d_{\text {max }}^{2}\right]\right\}$. The constants $|\partial \mathrm{B}|$ and $\grave{C}$ are defined in Proposition 2.7 and Proposition 2.10 respectively.

4. The constant $a_{0}$ appearing in (1.7) of Theorem 1.2 is the minimum among $\frac{1}{\omega_{\max }} \min \left\{c_{s}, c_{p}\right\}$, and $\frac{2 \sqrt{\pi} \max _{1 \leq m \leq M} \operatorname{diam}\left(B_{m}\right)}{\omega_{\max }\left(\left[\frac{4 \lambda+17 \mu}{2 c_{s}^{4}}+\frac{12 \lambda+9 \mu}{2 c_{p}^{4}}\right]|\partial \mathrm{B}| \max _{1 \leq m \leq M}\left\|\left(\frac{1}{2} I+\mathcal{D}_{B_{m}}^{i_{\omega}}\right)^{-1}\right\|_{\mathcal{L}\left(L^{2}\left(\partial B_{m}\right), L^{2}\left(\partial B_{m}\right)\right)}\right)^{\frac{1}{2}}}$.

5. The constant $c_{1}$ appearing in (1.12) of Theorem 1.2 is $\frac{5 \pi}{3} \frac{\mu}{(\lambda+2 \mu)^{2}} \frac{\min _{1 \leq m \leq M} C^{a}\left(B_{m}\right)}{\max _{1 \leq m \leq M} C^{a}\left(B_{m}\right)} \frac{\max _{1 \leq m \leq M} \operatorname{diam}\left(B_{m}\right)}{\max _{1 \leq m \leq M} C^{a}\left(B_{m}\right)}$ with $C^{a}\left(B_{m}\right)$ denoting the acoustic capacitance of the bodies $B_{m}$ and it follows from Corollary 3.3 and from [11, Lemma 2.11].

From the last points, we see that the constants appearing in Theorem 1.2 depend only on $d_{\max }, \omega_{\max }$, $\lambda, \mu$ and $B_{m}$ 's through their diameters, capacitances and the norms of the boundary operators $\mathcal{S}_{B_{m}}^{i_{\omega}}{ }^{-1}$ : $H^{1}\left(\partial B_{m}\right) \rightarrow L^{2}\left(\partial B_{m}\right),\left(\frac{1}{2} I+\mathcal{D}_{B_{m}}^{i_{\omega}}\right)^{-1}: L^{2}\left(\partial B_{m}\right) \rightarrow L^{2}\left(\partial B_{m}\right)$ and $\Lambda_{B_{m}}: H^{1}\left(\partial B_{m}\right) \rightarrow L^{2}\left(\partial B_{m}\right)$. As it was explained in the acoustic case in [11, Remark 2.23], the capacitances and the bounds of the operators $\mathcal{S}_{B_{m}}^{i_{\omega}}{ }^{-1}$ and $\left(\frac{1}{2} I+\mathcal{D}_{B_{m}}^{i_{\omega}}\right)^{-1}$ depend on $B_{m}$ 's actually only through their Lipschitz character.

\subsection{Proof of corollary 1.3}

For $m=1, \ldots, M$ fixed, we distinguish between the obstacles $D_{j}, j \neq m$ which are near to $D_{m}$ from the ones which are far from $D_{m}$ as follows. Let $\Omega_{m}, 1 \leq m \leq M$ be the balls of center $z_{m}$ and of radius $\left(\frac{a}{2}+d^{\alpha}\right)$ with $0<\alpha \leq 1$. The bodies lying in $\Omega_{m}$ will fall into the category, $N_{m}$, of near by obstacles and the others into the category, $F_{m}$, of far obstacles to $D_{m}$. Since the obstacles $D_{m}$ are balls with same diameter, the number of obstacles near by $D_{m}$ will not exceed $\left(\frac{a+2 d^{\alpha}}{a+d}\right)^{3}\left[=\frac{\frac{4}{3} \pi\left(\left(a+2 d^{\alpha}\right) / 2\right)^{3}}{\frac{4}{3} \pi((a+d) / 2)^{3}}\right]$.

With this observation, instead of (1.8) [1.9), the $\mathrm{P}$ and the S parts of the far field will have the asymptotic expansions (1.13-1.14). Indeed,

- For the bodies $D_{j} \in N_{m}, j \neq m$, we have the estimate (2.80) but for the bodies $D_{j} \in F_{m}$, we obtain the following estimate

$$
\left|R\left(s_{m}, s\right)\right| \leq \max _{y \in \bar{D}_{j}}\left|\nabla_{y} \Gamma^{\omega}\left(s_{m}, y\right)\right|<\frac{1}{4 \pi}\left[\frac{C_{9}}{d_{m j}^{2 \alpha}}+C_{10}\right]
$$

- Due to the estimates (2.80) and (2.105), corresponding changes will take place in (2.82, 2.84), (2.85), (2.92. 2.93) and in (2.97 2.98) which inturn modify (2.101-2.102) and hence the asymptotic expansion (1.8) as follows

$$
U_{p}^{\infty}(\hat{x}, \theta)=\frac{1}{4 \pi c_{p}^{2}}(\hat{x} \otimes \hat{x})\left[\sum_{m=1}^{M} e^{-i \frac{\omega}{c_{p}} \hat{x} \cdot z_{m}} Q_{m}+O\left(M a^{2}+M(M-1) \frac{a^{3}}{d^{2 \alpha}}+M\left(\frac{a+2 d^{\alpha}}{a+d}\right)^{3} \frac{a^{3}}{d^{2}}\right.\right.
$$




$$
\begin{aligned}
& +M(M-1)^{2} \frac{a^{4}}{d^{3 \alpha}}+M(M-1)\left(\frac{a+2 d^{\alpha}}{a+d}\right)^{3} \frac{a^{4}}{d^{2+\alpha}} \\
& \left.\left.+M(M-1)\left(\frac{a+2 d^{\alpha}}{a+d}\right)^{3} \frac{a^{4}}{d^{2 \alpha+1}}+M\left(\frac{a+2 d^{\alpha}}{a+d}\right)^{6} \frac{a^{4}}{d^{3}}\right)\right] .
\end{aligned}
$$

- Since $\kappa \leq \kappa_{\max }, d \leq d^{\alpha}, 0<\alpha \leq 1$ and $\frac{a}{d}<\infty$, we have

$$
\left(\frac{a+2 d^{\alpha}}{a+d}\right)=d^{\alpha-1} \frac{a d^{-\alpha}+2}{a d^{-1}+1}=O\left(d^{\alpha-1}\right)
$$

which can be used to derive (1.13) from (2.106). In the similar way, we can obtain (1.14). Finally, it is easily seen that the above analysis applies also for non-flat Lipschitz domains $D_{m}$ by using the double inclusions (1.17) and the fact that $t_{m}$ 's are uniformly bounded from below by a positive constant.

\section{Solvability of the linear-algebraic system (2.100)}

The main object of this section is to give a sufficient condition in order to get the invertibility of the linear algebraic system (2.100). To achieve this, first we state the following lemma which estimates the eigenvalues of the elastic capacitance matrix of each scatterer in terms of its acoustic capacitance.

Lemma 3.1. Let $\lambda_{e i g_{m}}^{\text {min }}$ and $\lambda_{e i g_{m}}^{\text {max }}$ be the minimal and maximal eigenvalues of the elastic capacitance matrices $\bar{C}_{m}$, for $m=1,2, \ldots, M$. Denote by $C_{m}^{a}$ the capacitance of each scatterer in the acoustic case 5 then we have the following estimate;

$$
\mu C_{m}^{a} \leq \lambda_{\text {eigm }}^{\min } \leq \lambda_{\text {eigm }}^{\max } \leq(\lambda+2 \mu) C_{m}^{a}, \quad \text { for } m=1,2, \ldots, M .
$$

Proof. of Lemma 3.1. Proof of this Lemma follows as in [22, Lemma 6.3.6]. See also [23, Lemma 10].

Now, we prove the main lemma of this section.

Lemma 3.2. The matrix $\mathbf{B}$ is invertible and the solution vector $\tilde{Q}$ of (2.100) satisfies the estimate:

$$
\sum_{m=1}^{M}\left\|\tilde{Q}_{m}\right\|_{2}^{2} \leq 4 \quad\left(\min _{m=1}^{M} \lambda_{\text {eig }_{m}}^{\min }-\frac{3 t}{5 \pi d} \max _{m=1}^{M} \lambda_{\text {eig }_{m}}^{\max ^{2}}\right)^{-2}\left(\max _{m=1}^{M} \lambda_{\text {eig }_{m}}^{\max }\right)^{4} \sum_{m=1}^{M}\left\|U^{i}\left(z_{m}\right)\right\|_{2}^{2},
$$

if we consider $\left(\max _{1 \leq m \leq M} \lambda_{\text {eigm }}^{m a x^{2}}\right)<t^{-1}\left(\frac{5 \pi}{3} d \min _{1 \leq m \leq M} \lambda_{\text {eigm }}^{\text {min }}\right)$ with the positively assumed value

$t:=\left[\frac{1}{c_{p}^{2}}-2 \operatorname{diam}(\Omega) \frac{\omega}{c_{s}^{3}}\left(\frac{1-\left(\frac{1}{2} \kappa_{s} \omega \operatorname{diam}(\Omega)\right)^{N_{\Omega}}}{1-\left(\frac{1}{2} \kappa_{s} \omega \operatorname{diam}(\Omega)\right)}+\frac{1}{2^{N_{\Omega^{-1}}}}\right)-\operatorname{diam}(\Omega) \frac{\omega}{c_{p}^{3}}\left(\frac{1-\left(\frac{1}{2} \kappa_{p} \omega \operatorname{diam}(\Omega)\right)^{N_{\Omega}}}{1-\left(\frac{1}{2} \kappa_{p} \omega \operatorname{diam}(\Omega)\right)}+\frac{1}{2^{N_{\Omega^{-1}}}}\right)\right]$.

Proof. of Lemma 3.2. We can factorize $\mathbf{B}$ as $\mathbf{B}=-\left(I+\mathbf{B}_{n} \mathbf{C}\right) \mathbf{C}^{-1}$ where $\mathbf{C}:=\operatorname{Diag}\left(\bar{C}_{1}, \bar{C}_{2}, \ldots, \bar{C}_{M}\right) \in$ $\mathbb{R}^{3 M \times 3 M}, I$ is the identity matrix and $\mathbf{B}_{n}:=-\mathbf{C}^{-1}-\mathbf{B}$. Hence, the solvability of the system (2.100), depends on the existence of the inverse of $\left(I+\mathbf{B}_{n} \mathbf{C}\right)$. We have $\left(I+\mathbf{B}_{n} \mathbf{C}\right): \mathbb{C}^{3 M} \rightarrow \mathbb{C}^{3 M}$, so it is enough to prove the injectivity in order to prove its invetibility. For this purpose, let $X, Y$ are vectors in $\mathbb{C}^{M}$ and consider the system

$$
\left(I+\mathbf{B}_{n} \mathbf{C}\right) X=Y
$$

\footnotetext{
${ }^{5}$ Recall that, for $m=1, \ldots, M, C_{m}^{a}:=\int_{\partial D_{m}} \sigma_{m}(s) d s$ and $\sigma_{m}$ is the solution of the integral equation of the first kind $\int_{\partial D_{m}} \frac{\sigma_{m}(s)}{4 \pi|t-s|} d s=1, t \in \partial D_{m}$, see [1].
} 
Let $(\cdot)^{\text {real }}$ and $(\cdot)^{i m g}$ denotes the real and the imaginary parts of the corresponding complex nump ber/vecctor/matrix. Now, the following can be written from (3.3);

$$
\begin{aligned}
& \left(I+\mathbf{B}_{n}^{\text {real }} \mathbf{C}\right) X^{\text {real }}-\mathbf{B}_{n}^{\text {img }} \mathbf{C} X^{i m g}=Y^{\text {real }}, \\
& \left(I+\mathbf{B}_{n}^{\text {real }} \mathbf{C}\right) X^{i m g}+\mathbf{B}_{n}^{\text {img }} \mathbf{C} X^{\text {real }}=Y^{\text {img }},
\end{aligned}
$$

which leads to

$$
\begin{aligned}
\left\langle\left(I+\mathbf{B}_{n}^{\text {real }} \mathbf{C}\right) X^{\text {real }}, \mathbf{C} X^{\text {real }}\right\rangle-\left\langle\mathbf{B}_{n}^{\text {img }} \mathbf{C} X^{i m g}, \mathbf{C} X^{\text {real }}\right\rangle & =\left\langle Y^{\text {real }}, \mathbf{C} X^{\text {real }}\right\rangle, \\
\left\langle\left(I+\mathbf{B}_{n}^{\text {real }} \mathbf{C}\right) X^{\text {img }}, \mathbf{C} X^{\text {img }}\right\rangle+\left\langle\mathbf{B}_{n}^{\text {img }} \mathbf{C} X^{\text {real }}, \mathbf{C} X^{\text {img }}\right\rangle & =\left\langle Y^{\text {img }}, \mathbf{C} X^{\text {img }}\right\rangle .
\end{aligned}
$$

By summing up (3.6) and (3.7) will give

$$
\begin{array}{r}
\left\langle X^{\text {real }}, \mathbf{C} X^{\text {real }}\right\rangle+\left\langle\mathbf{B}_{n}^{\text {real }} \mathbf{C} X^{\text {real }}, \mathbf{C} X^{\text {real }}\right\rangle+\left\langle X^{i m g}, \mathbf{C} X^{i m g}\right\rangle+\left\langle\mathbf{B}_{n}^{\text {real }} \mathbf{C} X^{i m g}, \mathbf{C} X^{i m g}\right\rangle \\
=\left\langle Y^{\text {real }}, \mathbf{C} X^{\text {real }}\right\rangle+\left\langle Y^{\text {img }}, \mathbf{C} X^{\text {img }}\right\rangle .
\end{array}
$$

Indeed,

$$
\left\langle\mathbf{B}_{n}^{i m g} \mathbf{C} X^{i m g}, \mathbf{C} X^{r e a l}\right\rangle=\left\langle\mathbf{C} X^{i m g}, \mathbf{B}_{n}^{i m g^{*}} \mathbf{C} X^{\text {real }}\right\rangle=\left\langle\mathbf{C} X^{i m g}, \mathbf{B}_{n}^{i m g} \mathbf{C} X^{\text {real }}\right\rangle=\left\langle\mathbf{B}_{n}^{\text {img }} \mathbf{C} X^{\text {real }}, \mathbf{C} X^{i m g}\right\rangle .
$$

We can observe that, the right-hand side in (3.8) does not exceed

$$
\begin{aligned}
\left\langle X^{\text {real }}, \mathbf{C} X^{\text {real }}\right\rangle^{1 / 2}\left\langle Y^{\text {real }}, \mathbf{C} Y^{\text {real }}\right\rangle^{1 / 2} & +\left\langle X^{i m g}, \mathbf{C} X^{i m g}\right\rangle^{1 / 2}\left\langle Y^{i m g}, \mathbf{C} Y^{i m g}\right\rangle^{1 / 2} \\
& \leq 2\left\langle X^{\|\cdot\|},(\mathbf{C} X)^{\|\cdot\|}\right\rangle^{1 / 2}\left\langle Y^{\|\cdot\|},(\mathbf{C} Y)^{\|\cdot\|}\right\rangle^{1 / 2}
\end{aligned}
$$


term in the left-hand side of (3.8). Using the mean value theorem for harmonic functions we deduce

$$
\begin{aligned}
\left\langle\mathbf{B}_{n}^{\text {real }} \mathbf{C} X^{\text {real }}, \mathbf{C} X^{\text {real }}\right\rangle & =\sum_{\substack{1 \leq j, m \leq M \\
j \neq m}} X_{m}^{\text {real }}{ }^{\top} \bar{C}_{m}^{\top}\left[\Gamma^{\omega}\left(z_{m}, z_{j}\right)\right]^{\text {real }} \bar{C}_{j} X_{j}^{\text {real }} \\
& \geq t \sum_{\substack{1 \leq j, m \leq M \\
j \neq m}} X_{m}^{r e a l} \bar{C}_{m}^{\top}\left(\frac{1}{\left|B^{(j)}\right|\left|B^{(m)}\right|} \int_{B^{(j)}} \int_{B^{(m)}} \Phi_{0}(x, y) d x d y\right) \bar{C}_{j} X_{j}^{\text {real }},
\end{aligned}
$$

Similarly, if we consider the fourth term in the left-hand side of (3.8), we deduce

$$
\begin{aligned}
\left\langle\mathbf{B}_{n}^{\text {real }} \mathbf{C} X^{i m g}, \mathbf{C} X^{i m g}\right\rangle & =\sum_{\substack{1 \leq j, m \leq M \\
j \neq m}} X_{m}^{i m g^{\top}} \bar{C}_{m}^{\top}\left[\Gamma^{\omega}\left(z_{m}, z_{j}\right)\right]^{\text {real }} \bar{C}_{j} X_{j}^{i m g} \\
& \geq t \sum_{\substack{1 \leq j, m \leq M \\
j \neq m}} X_{m}^{i m g^{\top}} \bar{C}_{m}^{\top}\left(\frac{1}{\left|B^{(j)}\right|\left|B^{(m)}\right|} \int_{B^{(j)}} \int_{B^{(m)}} \Phi_{0}(x, y) d x d y\right) \bar{C}_{j} X_{j}^{i m g},
\end{aligned}
$$

where

$$
t:=\left[\frac{1}{c_{p}^{2}}-2 \operatorname{diam}(\Omega) \frac{\omega}{c_{s}^{3}}\left(\frac{1-\left(\frac{1}{2} \kappa_{s} \omega \operatorname{diam}(\Omega)\right)^{N_{\Omega}}}{1-\left(\frac{1}{2} \kappa_{s} \omega \operatorname{diam}(\Omega)\right)}+\frac{1}{2^{N_{\Omega}-1}}\right)-\operatorname{diam}(\Omega) \frac{\omega}{c_{p}^{3}}\left(\frac{1-\left(\frac{1}{2} \kappa_{p} \omega \operatorname{diam}(\Omega)\right)^{N_{\Omega}}}{1-\left(\frac{1}{2} \kappa_{p} \omega \operatorname{diam}(\Omega)\right)}+\frac{1}{2^{N_{\Omega}-1}}\right)\right]
$$

assumed to be positive, $\Phi_{0}(x, y):=1 /(4 \pi|x-y|), x \neq y$ and $B^{(m)}:=\left\{x:\left|x-z_{m}\right|<d / 2\right\}, m=1, \ldots, M$, are non-overlapping balls of radius $d / 2$ with centers at $z_{m}$, and $\left|B^{(m)}\right|=\pi d^{3} / 6$ are the volumes of the balls. 
Also, we use the notation $B_{d}$ to denote the balls of radius $d / 2$ with the center at the origin. Indeed, we can write $\Gamma^{\omega}\left(z_{m}, z_{j}\right)$ from (2.3) as,

$$
\begin{aligned}
\Gamma^{\omega}\left(z_{m}, z_{j}\right)= & \frac{1}{4 \pi\left|z_{m}-z_{j}\right|}(\frac{1}{2}\left[\frac{1}{c_{s}^{2}}+\frac{1}{c_{p}^{2}}\right] \mathbf{I}+\underbrace{\frac{1}{2}\left[\frac{1}{c_{s}^{2}}-\frac{1}{c_{p}^{2}}\right] \frac{\left(z_{m}-z_{j}\right)}{\left|z_{m}-z_{j}\right|} \otimes \frac{\left(z_{m}-z_{j}\right)}{\left|z_{m}-z_{j}\right|}}_{\mathrm{b}_{\Gamma}} \\
& +\underbrace{\sum_{l=1}^{\infty} \frac{i^{l}}{l !(l+2)} \frac{1}{\omega^{2}}\left((l+1) \kappa_{s^{\omega}}^{l+2}+\kappa_{p^{\omega}}^{l+2}\right)\left|z_{m}-z_{j}\right|^{l} \mathbf{I}}_{c 1_{\Gamma}} \\
& \underbrace{-\sum_{l=1}^{\infty} \frac{i^{l}}{l !(l+2)} \frac{(l-1)}{\omega^{2}}\left(\kappa_{s}^{l+2}-\kappa_{p^{\omega}}^{l+2}\right)\left|z_{m}-z_{j}\right|^{l-2}\left(z_{m}-z_{j}\right) \otimes\left(z_{m}-z_{j}\right)}_{c 2_{\Gamma}})
\end{aligned}
$$

from which, we get the required result by estimating $\Gamma^{\omega}\left(z_{m}, z_{j}\right)$. Notice that

$$
\begin{aligned}
&\left|b_{\Gamma}\right| \leq \frac{1}{2}\left[\frac{1}{c_{s}^{2}}-\frac{1}{c_{p}^{2}}\right] \text { and } \\
&\left|c 1_{\Gamma}+c 2_{\Gamma}\right| \leq \sum_{l=1}^{\infty} \frac{1}{(l-1) !(l+2)} \frac{1}{\omega^{2}}\left(2 \kappa_{s^{\omega}}^{l+2}+\kappa_{p^{\omega}}^{l+2}\right)\left|z_{m}-z_{j}\right|^{l} \\
& \quad\left[\operatorname{By~recalling~} N_{\Omega}=\left[2 \operatorname{diam}(\Omega) \max \left\{\kappa_{s^{\omega}}, \kappa_{p^{\omega}}\right\} e^{2}\right]\right. \text { and using Lemma 2.6] } \\
& \leq \operatorname{diam}(\Omega)\left[\frac{2 \omega}{c_{s}^{3}}\left(\sum_{l=1}^{N_{\Omega}}\left(\frac{1}{2} \kappa_{s^{\omega}} \operatorname{diam}(\Omega)\right)^{l-1}+\sum_{l=N_{\Omega}+1}^{\infty} \frac{1}{2^{l-1}}\right)\right. \\
&\left.\quad+\frac{\omega}{c_{p}^{3}}\left(\sum_{l=1}^{N_{\Omega}}\left(\frac{1}{2} \kappa_{p^{\omega}} \operatorname{diam}(\Omega)\right)^{l-1}+\sum_{l=N_{\Omega}+1}^{\infty} \frac{1}{2^{l-1}}\right)\right] \\
& \quad \operatorname{diam}(\Omega)\left[2 \frac{\omega}{c_{s}^{3}}\left(\frac{1-\left(\frac{1}{2} \kappa_{s} \omega \operatorname{diam}(\Omega)\right)^{N_{\Omega}}}{1-\left(\frac{1}{2} \kappa_{s} \omega \operatorname{diam}(\Omega)\right)}+\frac{1}{2^{N_{\Omega}-1}}\right)\right. \\
&\left.+\frac{\omega}{c_{p}^{3}}\left(\frac{1-\left(\frac{1}{2} \kappa_{p^{\omega}} \operatorname{diam}(\Omega)\right)^{N_{\Omega}}}{1-\left(\frac{1}{2} \kappa_{p^{\omega}} \operatorname{diam}(\Omega)\right)}+\frac{1}{2^{N_{\Omega}-1}}\right)\right]
\end{aligned}
$$

Let $\Omega$ be a large ball with radius $R$. Also let $\Omega_{s} \subset \Omega$ be a ball with fixed radius $r(\leq R)$, which consists of all our small obstacles $D_{m}$ and also the balls $B^{(m)}$, for $m=1, \ldots, M$.

Let $\Upsilon^{\text {real }}(x)$ and $\Upsilon^{i m g}(x)$ be piecewise constant functions defined on $\mathbb{R}^{3}$ as

$$
\Upsilon^{\text {real }(\mathrm{img})}(x)=\left\{\begin{array}{cc}
\bar{C}_{m} X_{m}^{\text {real }(\mathrm{img})} & \text { in } B^{(m)}, \\
0 & \text { otherwise. }
\end{array}\right.
$$

Then

$$
\begin{aligned}
\left\langle\mathbf{B}_{n}^{\text {real }} \mathbf{C} X^{\text {real }}, \mathbf{C} X^{\text {real }}\right\rangle \geq \frac{36 t}{\pi^{2} d^{6}} & \left(\int_{\Omega} \int_{\Omega} \Phi_{0}(x, y) \Upsilon^{\text {real }}{ }^{\top}(x) \Upsilon^{\text {real }}(y) d x d y\right. \\
& \left.-\sum_{m=1}^{M}\left|\bar{C}_{m} X_{m}^{\text {real }}\right|^{2} \int_{B^{(m)}} \int_{B^{(m)}} \Phi_{0}(x, y) d x d y\right)
\end{aligned}
$$




$$
\begin{aligned}
\left\langle\mathbf{B}_{n}^{\text {real }} \mathbf{C} X^{i m g}, \mathbf{C} X^{i m g}\right\rangle \geq \frac{36 t}{\pi^{2} d^{6}} & \left(\int_{\Omega} \int_{\Omega} \Phi_{0}(x, y) \Upsilon^{i m g^{\top}}(x) \Upsilon^{i m g}(y) d x d y\right. \\
& \left.-\sum_{m=1}^{M}\left|\bar{C}_{m} X_{m}^{i m g}\right|^{2} \int_{B^{(m)}} \int_{B^{(m)}} \Phi_{0}(x, y) d x d y\right)
\end{aligned}
$$

Applying the mean value theorem to the harmonic function $\frac{1}{4 \pi|x-y|}$, as done in [21, p:109-110], we have the following estimate

$$
\int_{B^{(m)}} \int_{B^{(m)}} \Phi_{0}(x, y) d x d y=\frac{1}{4 \pi} \int_{B_{d}} \int_{B_{d}} \frac{1}{|x-y|} d x d y \leq \frac{\pi d^{5}}{60}
$$

Consider the first term in the right-hand side of (3.12), denote it by $A_{R}^{\text {real }}$, then by Green's theorem

$$
\begin{aligned}
A_{R}^{\text {real }}:= & \int_{\Omega} \int_{\Omega} \Phi_{0}(x, y) \Upsilon^{\text {real }}{ }^{\top}(x) \Upsilon^{\text {real }}(y) d x d y \\
= & \underbrace{\int_{\Omega}\left|\nabla_{x} \int_{\Omega} \Phi_{0}(x, y) \Upsilon^{\text {real }}(y) d y\right|^{2} d x}_{=: B_{R}^{\text {real }} \geq 0} \\
& -\underbrace{\int_{\partial \Omega}\left(\frac{\partial}{\partial \nu_{x}} \int_{\Omega} \Phi_{0}(x, y) \Upsilon^{\text {real }}(y) d y\right)^{\top}\left(\int_{\Omega} \Phi_{0}(x, y) \Upsilon^{\text {real }}(y) d y\right) d S_{x}}_{=: C_{R}^{\text {real }}} .
\end{aligned}
$$

We have

$$
\begin{aligned}
C_{R}^{r e a l} & =\int_{\partial \Omega}\left(\int_{\Omega} \frac{\partial}{\partial \nu_{x}} \Phi_{0}(x, y) \Upsilon^{\text {real }}(y) d y\right)^{\top}\left(\int_{\Omega} \Phi_{0}(x, y) \Upsilon^{\text {real }}(y) d y\right) d S_{x} \\
& =\int_{\partial \Omega}\left(\int_{\Omega_{s}} \frac{\partial}{\partial \nu_{x}} \Phi_{0}(x, y) \Upsilon^{\text {real }}(y) d y\right)^{\top}\left(\int_{\Omega_{s}} \Phi_{0}(x, y) \Upsilon^{\text {real }}(y) d y\right) d S_{x} \\
& =\int_{\partial \Omega}\left(\int_{\Omega_{s}} \frac{-(x-y)}{4 \pi|x-y|^{3}} \Upsilon^{\text {real }}(y) d y\right)^{\top}\left(\int_{\Omega_{s}} \frac{1}{4 \pi|x-y|} \Upsilon^{r e a l}(y) d y\right) d S_{x}
\end{aligned}
$$

which gives the following estimate;

$$
\begin{aligned}
\left|C_{R}^{r e a l}\right| & \leq \frac{1}{16 \pi^{2}} \int_{\partial \Omega} \frac{1}{|R-r|^{3}}\left(\int_{\Omega_{s}}\left|\Upsilon^{\text {real }}(y)\right| d y\right)^{2} d S_{x} \\
& \leq \frac{1}{16 \pi^{2}} \frac{1}{(R-r)^{3}} \int_{\partial \Omega}\left|\Omega_{s}\right|\left\|\Upsilon^{\text {real }}\right\|_{\mathcal{L}^{2}\left(\Omega_{s}\right)}^{2} d S_{x} \\
& =\frac{r^{3}}{12 \pi(R-r)^{3}} \sum_{m=1}^{M}\left|\bar{C}_{m} X_{m}^{\text {real }}\right|^{2}|\Omega| \\
& =\frac{R^{2} r^{3}}{3(R-r)^{3}} \sum_{m=1}^{M}\left|\bar{C}_{m} X_{m}^{\text {real }}\right|^{2} .
\end{aligned}
$$

Substitution of (3.17) in (3.15) gives

$$
\begin{aligned}
\int_{\Omega} \int_{\Omega} \Phi_{0}(x, y) \Upsilon^{\text {real }}(x) \Upsilon^{\text {real }}(y) d x d y \\
\geq \quad \int_{\Omega}\left|\nabla_{x} \int_{\Omega} \Phi_{0}(x, y) \Upsilon^{\text {real }}(y) d y\right|^{2} d x-\frac{R^{2} r^{3}}{3(R-r)^{3}} \sum_{m=1}^{M}\left|\bar{C}_{m} X_{m}^{\text {real }}\right|^{2}
\end{aligned}
$$


By considering the first term in the right-hand side of (3.13), and following the same procedure as mentione 35 in (3.15), (3.16) and (3.17), we obtain

$$
\begin{aligned}
& \int_{\Omega} \int_{\Omega} \Phi_{0}(x, y) \Upsilon^{i m g}(x) \Upsilon^{i m g}(y) d x d y \\
& \quad \geq \int_{\Omega}\left|\nabla_{x} \int_{\Omega} \Phi_{0}(x, y) \Upsilon^{i m g}(y) d y\right|^{2} d x-\frac{R^{2} r^{3}}{3(R-r)^{3}} \sum_{m=1}^{M}\left|\bar{C}_{m} X_{m}^{i m g}\right|^{2} .
\end{aligned}
$$

Under our assumption $t>0,(3.12),(3.13)$ (3.14), (3.18) and (3.19) lead to

$$
\begin{aligned}
& \left\langle\mathbf{B}_{n}^{\text {real }} \mathbf{C} X^{\text {real }}, \mathbf{C} X^{\text {real }}\right\rangle \\
& \quad \geq \frac{36 t}{\pi^{2} d^{6}}\left(\int_{\Omega}\left|\nabla_{x} \int_{\Omega} \Phi_{0}(x, y) \Upsilon^{\text {real }}(y) d y\right|^{2} d x-\left[\frac{R^{2} r^{3}}{3(R-r)^{3}}+\frac{\pi d^{5}}{60}\right] \sum_{m=1}^{M}\left|\bar{C}_{m} X_{m}^{\text {real }}\right|^{2}\right) \\
& \left\langle\mathbf{B}_{n}^{\text {real }} \mathbf{C} X^{i m g}, \mathbf{C} X^{i m g}\right\rangle \\
& \quad \geq \frac{36 t}{\pi^{2} d^{6}}\left(\int_{\Omega}\left|\nabla_{x} \int_{\Omega} \Phi_{0}(x, y) \Upsilon^{i m g}(y) d y\right|^{2} d x-\left[\frac{R^{2} r^{3}}{3(R-r)^{3}}+\frac{\pi d^{5}}{60}\right] \sum_{m=1}^{M}\left|\bar{C}_{m} X_{m}^{i m g}\right|^{2}\right)
\end{aligned}
$$

Then (3.8), (3.20) and (3.21) imply

$$
\begin{aligned}
\left(\min _{m=1}^{M} \lambda_{e i g_{m}}^{\min }-\frac{36 t}{\pi^{2} d^{6}}\right. & {\left.\left[\frac{R^{2} r^{3}}{3(R-r)^{3}}+\frac{\pi d^{5}}{60}\right] \max _{m=1}^{M} \lambda_{\text {eigm }}^{\max _{2}}\right) \sum_{m=1}^{M}\left\|X_{m}\right\|_{2}^{2} } \\
& \leq 2\left(\max _{m=1}^{M} \lambda_{\text {eig }}^{\max }\right)\left(\sum_{m=1}^{M}\left\|X_{m}\right\|_{2}^{2}\right)^{1 / 2}\left(\sum_{m=1}^{M}\left\|Y_{m}\right\|_{2}^{2}\right)^{1 / 2} .
\end{aligned}
$$

As we have $R$ arbitrary, by tending $R$ to $\infty$, we can write (3.22) as

$$
\left(\min _{m=1}^{M} \lambda_{\text {eig }}^{\min }-\frac{3 t}{5 \pi d} \max _{m=1}^{M} \lambda_{\text {eig }_{m}}^{\max _{2}}\right) \sum_{m=1}^{M}\left\|X_{m}\right\|_{2}^{2} \leq 2\left(\operatorname{m}_{m=1}^{M} \lambda_{\text {eig }}^{\max }\right)\left(\sum_{m=1}^{M}\left\|X_{m}\right\|_{2}^{2}\right)^{1 / 2}\left(\sum_{m=1}^{M}\left\|Y_{m}\right\|_{2}^{2}\right)^{1 / 2} .
$$

which yields

$$
\sum_{m=1}^{M}\left\|X_{m}\right\|_{2}^{2} \leq 4\left(\min _{m=1}^{M} \lambda_{e i g_{m}}^{\min }-\frac{3 t}{5 \pi d} \max _{m=1}^{M} \lambda_{e i g_{m}}^{\max ^{2}}\right)^{-2}\left(\max _{m=1}^{M} \lambda_{e i g_{m}}^{\max }\right)^{2} \sum_{m=1}^{M}\left\|Y_{m}\right\|_{2}^{2}
$$

Thus, if $\left(\max _{1 \leq m \leq M} \lambda_{e i g_{m}}^{m_{1} x^{2}}\right)<t^{-1}\left(\frac{5 \pi}{3} d \min _{1 \leq m \leq M} \lambda_{e i g_{m}}^{m i n}\right)$ then the matrix $\mathbf{B}$ in algebraic system (2.100) is invertible and the estimate (3.23) and so (3.2) holds.

Corollary 3.3. If $(\lambda+2 \mu)^{2}\left(\max _{1 \leq m \leq M} C_{m}^{a}\right)^{2}<t^{-1}\left(\frac{5 \pi}{3} \mu d \min _{1 \leq m \leq M} C_{m}^{a}\right)$, then the matrix $\mathbf{B}$ is invertible and the solution vector $\tilde{Q}$ of (2.100) satisfies the estimate:




Proof. of Corollary 3.3. Let us assume the condition $(\lambda+2 \mu)^{2}\left(\max _{1 \leq m \leq M} C_{m}^{a}\right)^{2}<t^{-1}\left(\frac{5 \pi}{3} \mu d \min _{1 \leq m \leq M} C_{m}^{a}\right)^{36}$, then from Lemma 3.1 the sufficient condition of Lemma 3.2 is satisfied and hence (3.2) holds. Now, by applying the norm inequalities to (3.2), we obtain

$$
\sum_{m=1}^{M}\left\|\tilde{Q}_{m}\right\|_{2} \leq 2 \quad\left(\min _{m=1}^{M} \lambda_{e i g_{m}}^{\min }-\frac{3 t}{5 \pi d} \max _{m=1}^{M} \lambda_{\text {eig }}^{\max _{m}}\right)^{-1}\left(\max _{m=1}^{M} \lambda_{\text {eig }}^{\max }\right)^{2} M \max _{m=1}^{M}\left\|U^{i}\left(z_{m}\right)\right\|_{2} .
$$

Now, again by applying Lemma 3.1 to the above inequality (3.26) gives the result $(3.25)$.

\section{Appendix}

The object of this section is to derive some used properties of the single layer operator $\mathcal{S}_{D_{\epsilon}}: L^{2}\left(\partial D_{\epsilon}\right) \rightarrow$ $H^{1}\left(\partial D_{\epsilon}\right)$ defined by

$$
\left(\mathcal{S}_{D_{\epsilon}} \psi\right)(x):=\int_{\partial D_{\epsilon}} \Gamma^{\omega}(x, y) \psi(y) d y .
$$

Lemma 4.1. There exists $\epsilon_{0}$ such that if $\epsilon<\epsilon_{0}$ then the operator $\mathcal{S}_{D_{\epsilon}}$ is invertible.

Proof. of Lemma 4.1. Proof of this Lemma follows as the one of Proposition 2.1.

Lemma 4.2. Let $\phi \in H^{1}\left(\partial D_{\epsilon}\right)$ and $\psi \in L^{2}\left(\partial D_{\epsilon}\right)$. Then,

$$
\begin{gathered}
\mathcal{S}_{D_{\epsilon}} \psi=\epsilon\left(\mathcal{S}_{B}^{\epsilon} \hat{\psi}\right)^{\vee}, \\
\mathcal{S}_{D_{\epsilon}}^{-1} \phi=\epsilon^{-1}\left(\mathcal{S}_{B}^{\epsilon}-1 \hat{\phi}\right)^{\vee}
\end{gathered}
$$

and

$$
\left\|\mathcal{S}_{D_{\epsilon}}^{-1}\right\|_{\mathcal{L}\left(H^{1}\left(\partial D_{\epsilon}\right), L^{2}\left(\partial D_{\epsilon}\right)\right)} \leq \epsilon^{-1}\left\|\mathcal{S}_{B}^{\epsilon-1}\right\|_{\mathcal{L}\left(H^{1}(\partial B), L^{2}(\partial B)\right)}
$$

with $\mathcal{S}_{B}^{\epsilon} \hat{\psi}(\xi):=\int_{\partial B} \Gamma^{\epsilon \omega}(\xi, \eta) \hat{\psi}(\eta) d \eta$.

Proof. of Lemma 4.2.

- We have,

$$
\begin{aligned}
\mathcal{S}_{D_{\epsilon}} \psi(x) & =\int_{\partial D_{\epsilon}} \Gamma^{\omega}(x, y) \psi(y) d y \\
& =\int_{\partial B} \frac{1}{\epsilon} \Gamma^{\epsilon \omega}(\xi, \eta) \psi(\epsilon \eta+z) \epsilon^{2} d \eta \\
& =\epsilon \mathcal{S}_{B}^{\epsilon} \hat{\psi}(\xi) .
\end{aligned}
$$

The above gives us (4.2).

- The following equalities, using (4.2),

$$
\mathcal{S}_{D_{\epsilon}}\left(\mathcal{S}_{B}^{\epsilon-1} \hat{\phi}\right)^{\vee}=\epsilon\left(\mathcal{S}_{B}^{\epsilon} \mathcal{S}_{B}^{\epsilon-1} \hat{\phi}\right)^{\vee}=\epsilon \hat{\phi}^{\vee}=\epsilon \phi
$$

provides us (4.3). 
- We have from the estimate,

$$
\begin{aligned}
& \left\|\mathcal{S}_{D_{\epsilon}}^{-1}\right\|_{\mathcal{L}\left(H^{1}\left(\partial D_{\epsilon}\right), L^{2}\left(\partial D_{\epsilon}\right)\right)} \quad:=\quad \operatorname{Sup}_{\phi(\neq 0) \in H^{1}\left(\partial D_{\epsilon}\right)} \frac{\left\|\mathcal{S}_{D_{\epsilon}}^{-1} \phi\right\|_{L^{2}\left(\partial D_{\epsilon}\right)}}{\|\phi\|_{H^{1}\left(\partial D_{\epsilon}\right)}} \\
& \underset{[2.22),(2.23)}{\leq} \sup _{\phi(\neq 0) \in H^{1}\left(\partial D_{\epsilon}\right)} \frac{\epsilon\left\|\left(\mathcal{S}_{D_{\epsilon}}^{-1} \phi\right)^{\wedge}\right\|_{L^{2}(\partial B)}}{\epsilon\|\hat{\phi}\|_{H^{1}(\partial B)}} \\
& \underset{\substack{=\\
(4.3)}}{\operatorname{Sup}_{\hat{\phi}}(\neq 0) \in H^{1}\left(\partial D_{\epsilon}\right)} \frac{\epsilon^{-1}\left\|\mathcal{S}_{B}^{\epsilon-1} \hat{\phi}\right\|_{L^{2}(\partial B)}}{\|\hat{\phi}\|_{H^{1}(\partial B)}} \\
& =\quad \epsilon^{-1}\left\|\mathcal{S}_{B}^{\epsilon-1}\right\|_{\mathcal{L}\left(H^{1}(\partial B), L^{2}(\partial B)\right)} .
\end{aligned}
$$

\section{References}

[1] C. J. S. Alves and R. Kress. On the far-field operator in elastic obstacle scattering. IMA J. Appl. Math., 67(1):1-21, 2002.

[2] H. Ammari, H. Kang, G. Nakamura, and K. Tanuma, Complete asymptotic expansions of solutions of the system of elastostatics in the presence of an inclusion of small diameter and detection of an inclusion. J. Elasticity 67 (2002), no. 2, 97129 (2003)

[3] H. Ammari and H. Kang. Polarization and moment tensors. With applications to inverse problems and effective medium theory. Applied Mathematical Sciences, 162. Springer, New York, 2007. x+312 pp. ISBN: 978-0-387-71565-0

[4] H. Ammari; H. Kang; E. Kim and M. Lim. Reconstruction of closely spaced small inclusions. SIAM J. Numer. Anal. 42 (2005), no. 6, 2408-2428

[5] H. Ammari, P. Calmon, and E. Iakovleva. Direct elastic imaging of a small inclusion. SIAM J. Imaging Sci., 1(2):169-187, 2008.

[6] H. Ammari, H. Kang, and H. Lee. Asymptotic expansions for eigenvalues of the Lamé system in the presence of small inclusions. Comm. Partial Differential Equations, 32(10-12):1715-1736, 2007.

[7] H. Ammari, E. Bretin, J. Garnier, W. Jing, H. Kang, and A. Wahab, Localization, stability, and resolution of topological derivative based imaging functionals in elasticity. To appear in SIAM Journal on Imaging Sciences ( http://fr.arxiv.org/pdf/1210.6760).

[8] H. Ammari J. Garnier, W. Jing, H. Kang, M. Lim, K. Solna, and H. Wang. Mathematical and Statistical Methods for Multistatic Imaging. Lecture Notes in Mathematics, Volume 2098, Springer-Verlag, Berlin, 2013.

[9] A. Bensoussan; J. L. Lions and G. Papanicolaou. Asymptotic analysis for periodic structures. Studies in Mathematics and its Applications, 5. North-Holland Publishing Co., Amsterdam-New York, 1978.

[10] M. Cassier and C. Hazard. Multiple scattering of acoustic waves by small sound-soft obstacles in two dimensions: mathematical justification of the Foldy-Lax model. Wave Motion 50 (2013), no. 1, 18-28.

[11] D. P. Challa and M. Sini. On the justification of the Foldy-Lax approximation for the acoustic scattering by small rigid bodies of arbitrary shapes. Multiscale Model. Simul. 12 (2014), no. 1, 55108. 
[12] D. Colton and R. Kress. Inverse acoustic and electromagnetic scattering theory, volume 93 of Applie Mathematical Sciences. Springer-Verlag, Berlin, second edition, 1998.

[13] D. L. Colton and R. Kress. Integral equation methods in scattering theory. Pure and Applied Mathematics (New York). John Wiley \& Sons Inc., New York, 1983. A Wiley-Interscience Publication.

[14] V. Jikov, S. Kozlov and O. Oleinik. Homogenization of differential operators and integral functionals. Springer-Verlag, 1994.

[15] G. Hu and M. Sini. Elastic scattering by finitely many point-like obstacles. J. Math. Phys. 54 (2013), no. 4, 042901, $16 \mathrm{pp}$.

[16] V. D. Kupradze. Potential methods in the theory of elasticity. Israel Program for Scientific Translations, Jerusalem, 1965.

[17] V. D. Kupradze, T. G. Gegelia, M. O. Basheleĭshvili and T. V. Burchuladze. Three-dimensional problems of the mathematical theory of elasticity and thermoelasticity. North-Holland Publishing Co., Amsterdam, 1979.

[18] V. Marchenko and E. Khruslov Homogenization of partial differential equations. Birkhauser-Boston, 2006.

[19] P. A. Martin, Multiple scattering, Encyclopedia of Mathematics and its Applications, Volume 107 (Cambridge: Cambridge University Press), 2006.

[20] S. Mayboroda and M. Mitrea. The Poisson problem for the Lamé system on low-dimensional Lipschitz domains. In Integral methods in science and engineering, pages 137-160. Birkhäuser Boston, Boston, MA, 2006.

[21] V. Maz'ya and A. Movchan. Asymptotic treatment of perforated domains without homogenization. Math. Nachr., 283(1):104-125, 2010.

[22] V. Maz'ya, A. Movchan, and M. Nieves. Green's Kernels and Meso-Scale Approximations in Perforated Domains, volume 2077 of Lecture Notes in Mathematics. Springer-Verlag, Berlin, 2013.

[23] V. Maz'ya, A. Movchan, and M. Nieves. Uniform asymptotic formulae for Green's tensors in elastic singularly perturbed domains. Asymptot. Anal., 52(3-4):173-206, 2007.

[24] V. Maz'ya; A. Movchan and M. Nieves. Mesoscale asymptotic approximations to solutions of mixed boundary value problems in perforated domains. Multiscale Model. Simul. 9 (2011), no. 1, 424-448.

[25] W. McLean. Strongly elliptic systems and boundary integral equations. Cambridge University Press, Cambridge, 2000.

[26] D. Mitrea. The method of layer potentials for non-smooth domains with arbitrary topology. Integral Equations Operator Theory, 29(3):320-338, 1997.

[27] O. Mendez and M. Mitrea. The Banach envelopes of Besov and Triebel-Lizorkin spaces and applications to partial differential equations. J. Fourier Anal. Appl., 6(5):503-531, 2000.

[28] S. A. Nazarov, and J. Sokolowski. Self-adjoint extensions for the Neumann Laplacian and applications. Acta Math. Sin. (Engl. Ser.) 22 (2006), no. 3, 879906.

[29] A. G. Ramm. Wave scattering by small bodies of arbitrary shapes. World Scientific Publishing Co. Pte. Ltd., Hackensack, NJ, 2005.

[30] A. G. Ramm. Many-body wave scattering by small bodies and applications. J. Math. Phys. 48 (2007), no. 10, 103511, 29 pp. 
[31] A. G. Ramm. Wave scattering by small bodies and creating materials with a desired refraction coefficient. Afr. Mat. 22 (2011), no. 1, 3355.

[32] V. Namias. A simple derivation of Stirling's asymptotic series. Amer. Math. Monthly, 93(1):25-29, 1986. 